\title{
Onset Detection for Surface Electromyography Signals
}

By

Fang Dai, Bachelor of Science in Electrical Engineering

\author{
A thesis submitted to \\ the Faculty of Graduate Studies and Research \\ in partial fulfillment of \\ the requirements for the degree of \\ Master of Applied Science in Electrical and Computer Engineering \\ Ottawa-Carleton Institute for \\ Electrical and Computer Engineering (OCIECE) \\ Department of Systems and Computer Engineering \\ Carleton University \\ Ottawa, Ontario, Canada, K1S 5B6
}

November 2009

(C) Copyright 2009, Fang Dai 


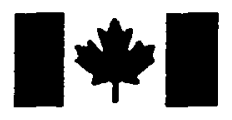

\author{
Library and Archives \\ Canada \\ Published Heritage \\ Branch \\ 395 Wellington Street \\ Ottawa ON K1A ON4 \\ Canada
}

Bibliothèque et

Archives Canada

Direction du

Patrimoine de l'édition

395 , rue Wellington

Ottawa ON K1A ON4

Canada
Your file Votre référence
ISBN: $978-0-494-63817-0$
Our file Notre reférence
ISBN: $978-0-494-63817-0$
NOTICE:

The author has granted a nonexclusive license allowing Library and Archives Canada to reproduce, publish, archive, preserve, conserve, communicate to the public by telecommunication or on the Internet, loan, distribute and sell theses worldwide, for commercial or noncommercial purposes, in microform, paper, electronic and/or any other formats.

The author retains copyright ownership and moral rights in this thesis. Neither the thesis nor substantial extracts from it may be printed or otherwise reproduced without the author's permission.
AVIS:

L'auteur a accordé une licence non exclusive permettant à la Bibliothèque et Archives Canada de reproduire, publier, archiver, sauvegarder, conserver, transmettre au public par télécommunication ou par l'Internet, prêter, distribuer et vendre des thèses partout dans le monde, à des fins commerciales ou autres, sur support microforme, papier, électronique et/ou autres formats.

L'auteur conserve la propriété du droit d'auteur et des droits moraux qui protège cette thèse. $\mathrm{Ni}$ la thèse ni des extraits substantiels de celle-ci ne doivent être imprimés ou autrement reproduits sans son autorisation.
In compliance with the Canadian Privacy Act some supporting forms may have been removed from this thesis.

While these forms may be included in the document page count, their removal does not represent any loss of content from the thesis.
Conformément à la loi canadienne sur la protection de la vie privée, quelques formulaires secondaires ont été enlevés de cette thèse.

Bien que ces formulaires aient inclus dans la pagination, il n'y aura aucun contenu manquant.

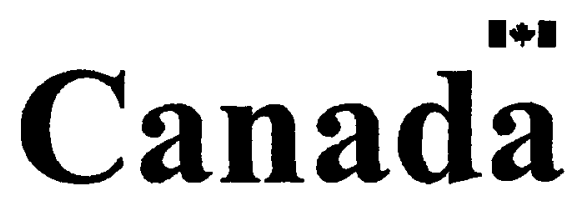




\section{Abstract}

Electromyography (EMG) has attracted attention in both academic research and medical diagnosis, due to its wide application in neurology, ergonomics, and physiology. There are intramuscularly recorded needle EMG signals and surface recorded EMG signals. In this work, we focus on the surface EMG (SEMG) signal.

Onset detection for SEMG signal is important due to its various applications. In this work, we reviewed four popular onset detection approaches: Hodges, Bonato, Lidierth, and Abbink; and evaluate their performance using real and synthetic SEMG signals. We analyzed the influence of high pass filters to the onset detection accuracy. We also propose a simple and generally applicable mechanism to determine an optimal threshold for various detection functions. Experimental results demonstrate that the proposed mechanisms (high pass filtering and a method for determining an optimal threshold) greatly improve the onset detection accuracy, which is comparable to manual onset detection. 


\section{Acknowledgements}

This research project would not have been possible without the support of many people.

The author wishes to express her sincere and deepest gratitude to her supervisor, Dr. Adrian D. C. Chan, who was abundantly helpful and offered invaluable assistance and consistent support. Without his technical guidance and financial support during the course of this project, this study would not have been successfully finished.

Special thanks are also sent to all her graduate group members: Mehran Talebinejad, Geoffrey Green, Beverly Bradley, for sharing the literature and invaluable assistance. In particular, the real data collected by Aarti Goge is indispensable for this work.

The author would also like to convey thanks to the Faculty of the Department of Systems and Computer Engineering of Carleton University, the Ottawa-Carleton Institute for Electrical and Computer Engineering (OCIECE) for providing the selfless assistance and laboratory facilities.

The author wishes to express her love and gratitude to her beloved families, for their understanding, endless love and full support, through the duration of her studies. 


\section{Table of Contents}

$\begin{array}{ll}\text { Abstract } & \text { iii }\end{array}$

Acknowledgements $\quad$ iv

$\begin{array}{ll}\text { Table of Contents } & \text { v }\end{array}$

List of Tables $\quad$ viii

List of Figures $\quad$ ix

Nomenclature $\quad$ xiii

$\begin{array}{lll}\text { Chapter } 1 & \text { Introduction } & 1\end{array}$

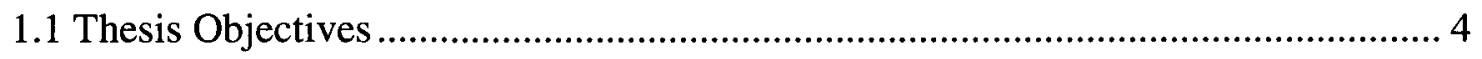

1.2 Thesis Contributions .................................................................................... 4

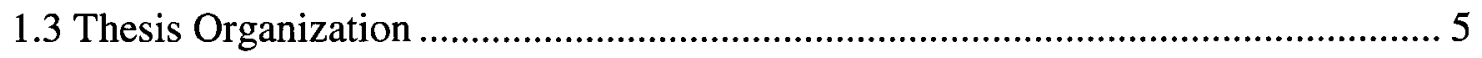

$\begin{array}{lll}\text { Chapter } 2 & \text { Background } & 7\end{array}$

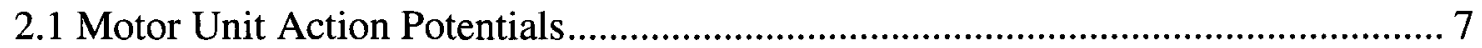

2.2 EMG Signal Generation/Collection ......................................................... 8

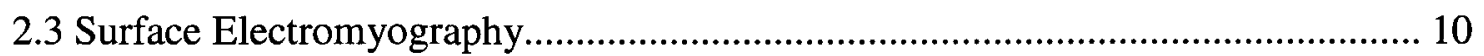

2.4 Observations in Electromyography Data Recording ...................................... 12

2.5 Electromyography Data Modeling................................................................. 13

2.5.1 Descriptive Models ....................................................................... 14

2.5.2 Structure-based Models ....................................................................... 14

2.5.3 Phenomenological Models .................................................................. 15

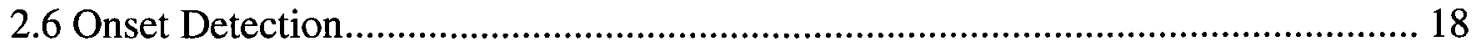




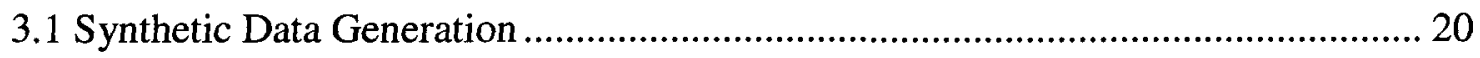

3.1.1 Synthetic SEMG Generation...................................................................... 20

3.1.2 Random SNR and Random Transition Time ................................................... 25

3.1.3 Random SNR and Constant Transition Time ……....................................... 27

3.1.4 Constant SNR and Random Transition Time ................................................. 27

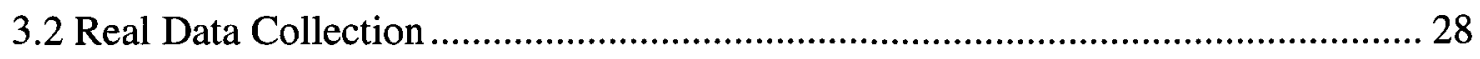

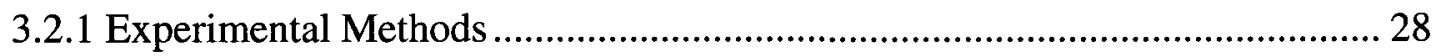

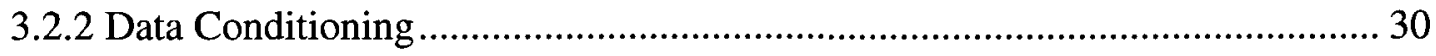

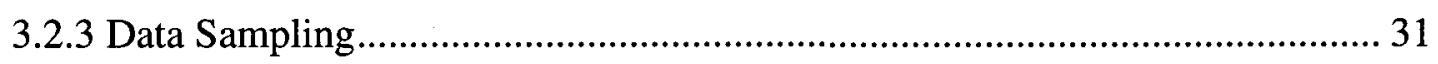

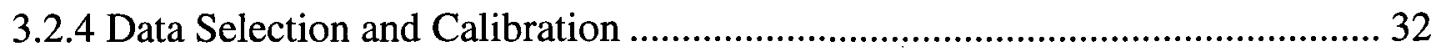

$\begin{array}{lll}\text { Chapter } 4 & \text { Onset Detection of SEMG Signals } & 34\end{array}$

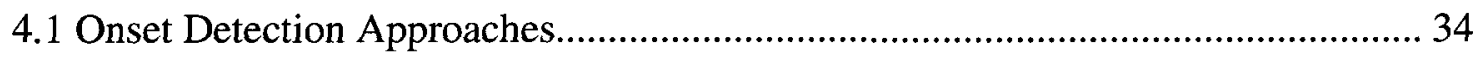

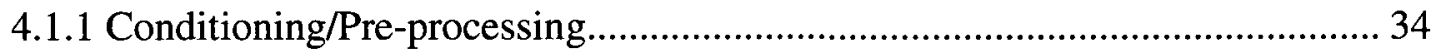

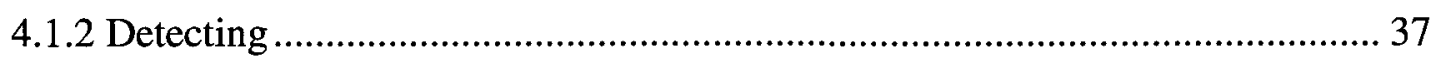

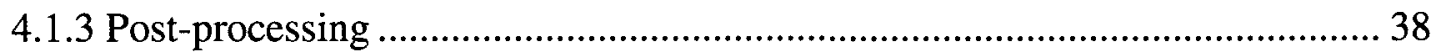

4.2 Algorithms and Implementation ....................................................................... 38

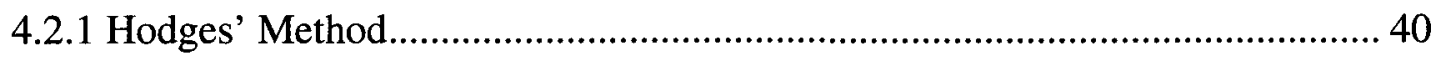

4.2.2 Bonato Method................................................................................................ 41

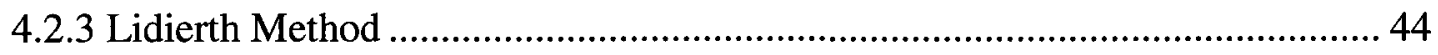

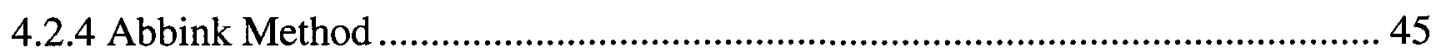

4.3 Algorithm Evaluation on Synthetic data................................................................ 47

4.3.1 Parameter Settings in Approaches ........................................................... 47

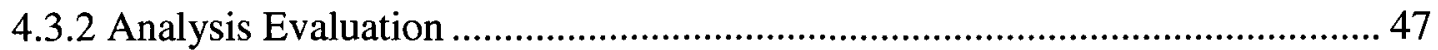

4.3.3 Evaluation on Synthetic Data..................................................................... 49

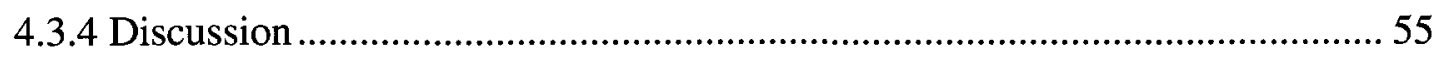

4.4 Algorithm Evaluation on Real Data................................................................. 56

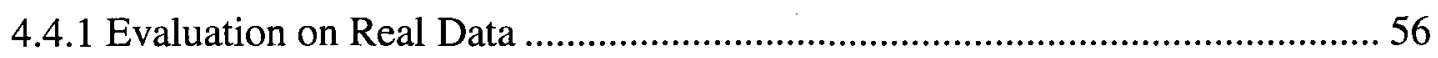




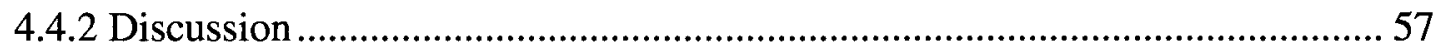

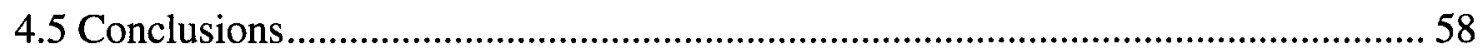

$\begin{array}{llr}\text { Chapter } 5 & 59\end{array}$

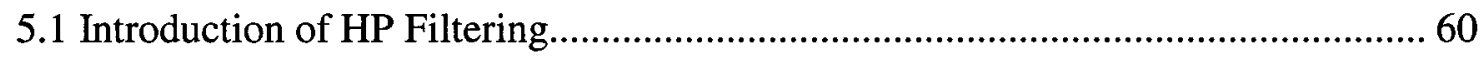

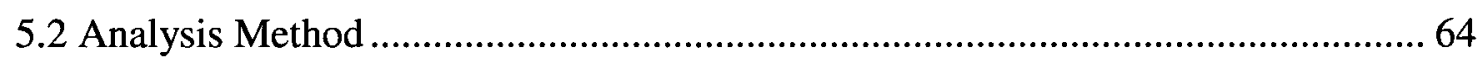

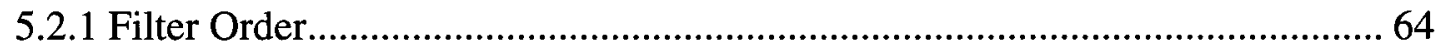

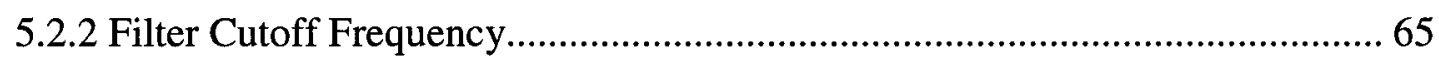

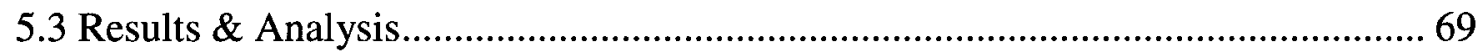

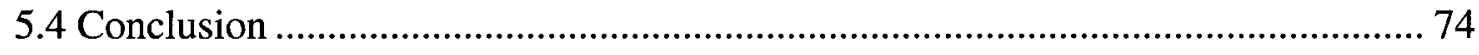

$\begin{array}{lll}\text { Chapter } 6 & \text { Optimal Threshold Selection } & 75\end{array}$

6.1 Influence of Threshold to Detection Accuracy ……............................................... 75

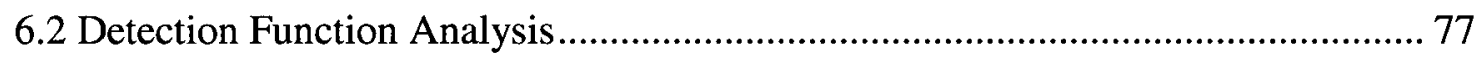

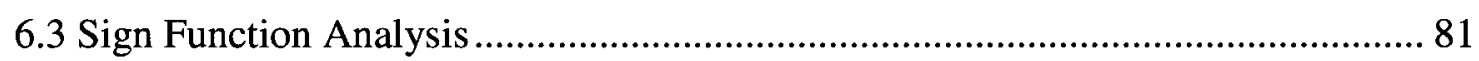

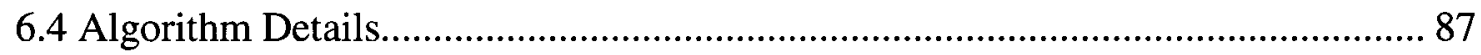

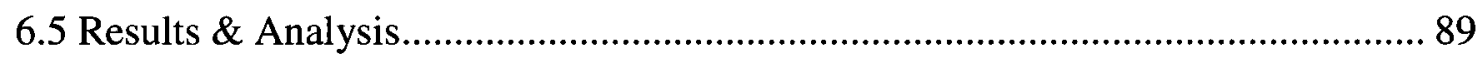

6.5.1 Results using Synthetic Data ................................................................... 90

6.5.2 Results using Real SEMG Data ...................................................................... 91

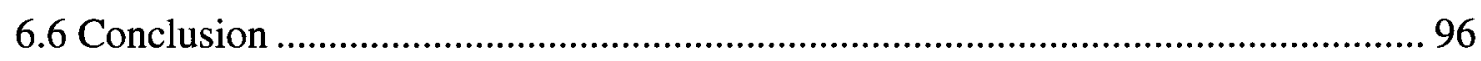

$\begin{array}{lll}\text { Chapter } 7 & \text { Conclusions and Future Work } & 98\end{array}$

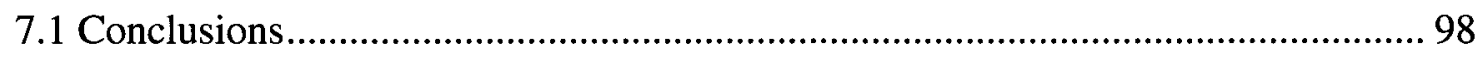

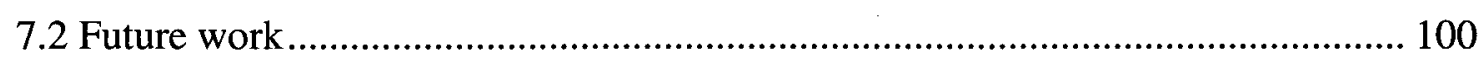

$\begin{array}{lr}\text { References } & 102\end{array}$ 


\section{List of Tables}

Table 4.1: More comparison results of detection error for four approaches on synthetic data. 50

Table 4.2: More comparison results of detection error for four approaches on real data. 57 Table 5.1: More comparison results of detection error for four approaches using HPF. . 67 Table 5.2: More comparison results of detection error for four approaches using $\mathrm{HPF}+\mathrm{LPF}$ 68

Table 5.3: Best cutoff frequency for each judging function. ........................................... 71

Table 5.4: Second level composition for best cutoff frequency for Lidierth approach.... 72 Table 5.5: More metrics on detection error for four approaches by using optimal HPF.. 73

Table 6.1: More metrics on detection error for four approaches on synthetic data.......... 90

Table 6.2: More metrics on detection error using optimal threshold w/o HPF. ................ 94

Table 6.3: More metrics on detection error using optimal threshold with HPF. 95 


\section{List of Figures}

Figure 2.1: A schematic representation of a motor unit, based on [65]....................... 8

Figure 2.2 A schematic representation of a MUAP [20] ......................................... 9

Figure 2.3 Bipolar detection of EMG signal. Source: [44] ........................................ 12

Figure 2.4 Synthetic data generation using frequency-domain approaches, base on [11].17

Figure 2.5 Synthetic data generation using time-domain approaches, based on [13]. .... 18

Figure 3.1 The general model to generate synthetic data, based on [64]...................... 21

Figure 3.2 Synthetic SEMG signal, with $\mathrm{SNR}=12 \mathrm{~dB}$ and transition time $22 \mathrm{~ms}$............ 22

Figure 3.3 Magnitude and phase response of the shaping filter. ................................. 23

Figure 3.4 The dataset (a) before and (b) after the shaping filter. ............................... 24

Figure 3.5 Procedures to generate synthetic signal................................................ 25

Figure 3.6 (a) SNR and (b) transition time in Random SNR and Random Transition Time.

Figure 3.7 Synthetic SEMG signal, with $\mathrm{SNR}=7 \mathrm{~dB}$ and transition time=13ms............ 26

Figure 3.8 Examples of Random SNR and Constant Transition Time dataset, with (a)

$\mathrm{SNR}=6 \mathrm{~dB},(\mathrm{~b}) \mathrm{SNR}=10 \mathrm{~dB}$.

Figure 3.9 Examples of Constant SNR and Random Transition Time, with transition time

(a) $\mathrm{t}=8 \mathrm{~ms}$ and (b) $\mathrm{t}=22 \mathrm{~ms}$. 28

Figure 3.10 Electrode placements from four perspectives...................................... 30

Figure 3.11 (a) Wrist flexion and (b) Channel 5 to collect data................................ 32

Figure 3.12 Histogram of reference onset time. ................................................... 33

Figure 4.1 The PSD of shaping filter and real EMG signal.................................... 36

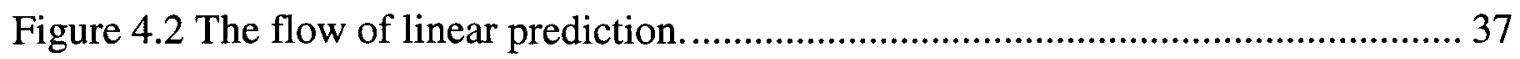

Figure 4.3 Implementation of Hodges' approach. .................................................. 41

Figure 4.4 Conditioning stage in Bonato method. .................................................... 42

Figure 4.5 Detection \& post-processing of Bonato method. .................................... 44 
Figure 4.6 Detection \& post-processing for Abbink

Figure 4.7 (a) Accuracy function and (b) normalized histograms of all approaches on synthetic data. 50

Figure 4.8 Accuracy function of four approaches using the original processes.

Figure 4.9 Accuracy function of four approaches using original parameter settings...... 52

Figure 4.10 Effect of SNR to the mean of (a) onset error and (b) absolute onset error.... 53

Figure 4.11 Effect of SNR to the s.t.d. of (a) onset error and (b) absolute onset error..... 53

Figure 4.12 Effect of ramp duration to mean of (a) onset error and (b) absolute onset error.

Figure 4.13 Effect of ramp duration to s.t.d. of (a) onset error and (b) absolute onset error.

Figure 4.14 (a) Accuracy function and (b) normalized histograms of all approaches on real data. 56

Figure 5.1 Magnitude and phase of the high pass filter.

Figure 5.2 (a) Raw and (b) filtered SEMG signal with a $6^{\text {th }}$ Butterworth HPF with cutoff frequency $20 \mathrm{~Hz}$. 62

Figure 5.3 (a) Raw and (b) HP filtered synthetic data with a $6^{\text {th }}$ Butterworth HPF with cutoff frequency $20 \mathrm{~Hz}$.

Figure 5.4 Magnitude and phase for high pass butter worth filter, with (a) order=1, cutoff $=20 \mathrm{~Hz}$; (b) order $=6$, cutoff $=20 \mathrm{~Hz}$; (c) order $=1$, cutoff $=400 \mathrm{~Hz}$; (d) order $=6$, cutoff $=400 \mathrm{~Hz}$. 65

Figure 5.5 (a) Accuracy function and (b) normalized histograms of all approaches using HPF in conditioning system. 66

Figure 5.6 (a) Accuracy function and (b) normalized histograms of all approaches using HPF and extra LPF in conditioning system. 68

Figure 5.7 The influence of cutoff frequency on the detection rate. 69

Figure 5.8 The influence of cutoff frequency on the (a) mean and (b) standard deviation of the onset detection error. 
Figure 5.9 The influence of cutoff frequency on the (a) skewness and (b) $P_{100}$ of the onset detection error.

Figure 5.10 (a) Accuracy function and (b) normalized histograms of all approaches with HPF pre-conditioning.

Figure 6.1 An example of the influence of threshold on (a) onset detection accuracy and (b) HPF filtered detection error. 76

Figure 6.2 A typical example of detection function for (a) Hodges' approach, (b) Bonato approach, (c) Lidierth approach, (d) Abbink approach................................................. 78 Figure 6.3 The filtered result of the detection function using different low pass filters... 79 Figure 6.4 Magnitude and phase response for (a) MA filter, (b) $6^{\text {th }}$ order Butterworth LP with cutoff $3 \mathrm{~Hz}$, (c) $6^{\text {th }}$ order Butterworth LP with cutoff $50 \mathrm{~Hz}$. 80

Figure 6.5 An example of (a) detection function and (b) its sign function of threshold $\mathrm{h}_{0}$.

Figure 6.6 Another example of (a) detection function $g_{k}$ and (b) its sign function. This is based on the $9^{\text {th }}$ segment of real data in Abbink approach.

Figure 6.7 Another example of (a) detection function $\mathrm{g}_{\mathrm{k}}$ and (b) its sign function. This is based on the $22^{\text {th }}$ segment of real data in Hodges approach.

Figure 6.8 Another example of (a) detection function $\mathrm{g}_{\mathrm{k}}$ and (b) its sign function. This is based on the $46^{\text {th }}$ segment of real data in Lidierth approach. 83

Figure 6.9 Case 1: The number of ups and that of downs are same. 83

Figure 6.10 A special case of Case 1: The number of ups and that of downs equal to 1.84 Figure 6.11 Case 2: The number of ups is more than that of downs.

Figure 6.12 A special case of Case 2: The number of ups equals to 1 and that of downs equals to zero.

Figure 6.13 Case 3: Two examples of sign function between detection function and the failed thresholds. .85

Figure 6.14 (a) The detection function and (b) its sign function. This is based on the $25^{\text {th }}$ segment real data for Hodges' approach. 86

Figure 6.15 Comparison between (a) raw signal and (b) high pass filtered $25^{\text {th }}$ set data. 87 
Figure 6.16 (a) Detection function $\mathrm{g}_{\mathrm{k}}$ of Hodges' approach using the HP filtered signal

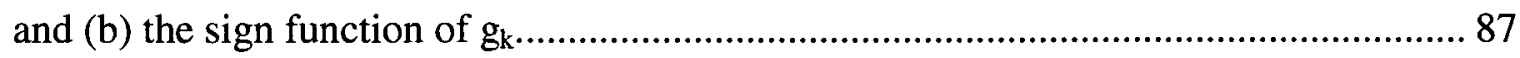

Figure 6.17 Optimal threshold decision algorithm. .......................................................... 89

Figure 6.18 (a) Accuracy function and (b) normalized histograms of detection error of four approaches with optimal threshold on synthetic data................................................... 90 Figure 6.19 (a) Optimal threshold selection and (b) the relation between the detected onset time and thresholds in Hodge's approach, with detected onset time 1149 ms........ 92 Figure 6.20 (a) Optimal threshold selection and (b) the relation between the detected onset time and thresholds in Bonato's approach, with detected onset time $1038 \mathrm{~ms}$....... 92 Figure 6.21 (a) Optimal threshold selection and (b) the relation between the detected onset time and thresholds in Lidierth's approach, with detected onset time 1008 ms...... 93 Figure 6.22 (a) Optimal threshold selection and (b) the relation between the detected onset time and thresholds in Abbink's approach, with detected onset time 954 ms. ....... 93 Figure 6.23 (a) Accuracy function and (b) normalized histograms of detection error of four approaches using optimal threshold w/o HPF on real data. .94 Figure 6.24 (a) Accuracy function and (b) normalized histograms of detection error of four approaches using optimal threshold with HPF on real data. 


\section{Nomenclature}

AP action potential

AR autoregressive

ARV average rectified value

ARMA autoregressive moving average

BPF band pass filter

CE correlation-ergodic

ECG electrocardiogram

EEG electroencephalogram

EMG electromyography

FCU flexor carpi ulnaris

FIR finite impulse response

FMA finite moving average

HPF high pass filter

IAP intracellular muscle-fiber action potential

IPI inter pulse intervals

LPF low pass filter

LTI linear, time-invariance

MA moving average

MAV mean absolute value 


\begin{tabular}{|c|c|}
\hline MDF & median frequency \\
\hline MNF & mean frequency \\
\hline MU & motor unit \\
\hline MUAP & motor unit action potential \\
\hline MVC & maximum voluntary contraction \\
\hline RMS & root-mean-square \\
\hline RT & reaction time \\
\hline PSD & power spectral density \\
\hline $\mathrm{PDF}$ & probability density function \\
\hline SEMG & surface EMG signal \\
\hline SENIAM & surface electromyography for non-invasive assessment of muscle \\
\hline SFAP & single fiber action potential \\
\hline SNR & signal-to noise ratios \\
\hline S.T.D. & standard deviation \\
\hline WSS & wide sense stationary \\
\hline
\end{tabular}




\section{Chapter 1 Introduction}

Biomedical signals, such as the electrocardiogram (ECG), electroencephalogram (EEG), and electromyogram, have attracted extensive interest in both research and clinical practice. From a research perspective, biomedical signals help the understanding of the human body mechanism, such as how specific phenomena or actions could generate these signals and how these signals reflect certain phenomena. From clinical perspective, biomedical signals build a bridge between certain phenomena and its identification (e.g. diagnoses of diseases). Electromyography (EMG) signals are biomedical signals that attract a great deal of attention due to its wide application in various fields such as neurology, ergonomics, and physiology.

The EMG signal is associated with the electrical activity of muscle fibers active during a contraction [44]. A motor unit (MU) is defined as a system comprised of an $\alpha$ motoneuron and the muscle fibers it innervates. It is well documented that $\mathrm{MU}$ recruitment and firing frequency directly reflect the level of force and the speed of contraction, which also indicate the change in EMG signals [44]; however, the EMG signal is further influenced by the individual muscle fiber potential, degree of MU discharge synchronization, and fatigue [4], [35]. MUs differ based on physiological properties such as speed of contraction and sensitivity to fatigue, and the number of MUs per muscle in humans has a wide range among different muscles [30]. 
In general, there are two ways to collect/record the EMG signal: using needle or using surface electrodes. Accordingly, there are two types of EMG signals: intramuscular EMG signal (i.e., needle EMG) and surface EMG signal (SEMG). The signal sources are located at the depolarized zones of the muscle fibers and are separated from the recording electrodes by biological tissues, referred to as volume conductor. The properties of this volume conductor largely determine the statistical characteristics of the detected signals, such as frequency and the boundary distance to detect signal. For the intramuscular signal, the effect of the tissues between electrodes and muscle fibers is relatively small due to the proximity of the recording electrodes to the sources. For surface EMG signals, the volume conductor acts as a spatial low-pass filter to the EMG signal [2]. In this thesis, we focus on the collection and analysis of SEMG signals.

The importance of recording SEMG signal can not be beyond that of processing SEMG, which is considered a special field of applied signal processing, with the main focus on applying theoretical concepts to extract specific information from small and often noisy biosignals [64]. When processing SEMG signal, the determination of exact onset time of a muscle contraction is very useful in studies of motor control and performance. In the past, onset time is often visually monitored and identified. However, this kind of manual detection heavily depends on subjective factors such as the experience of human inspectors and has large variation due to the inter and intra variability of the human inspectors. Thus, an automatic onset time detector is highly preferred [10], [45], [64]. 
The usage of computer is inevitable for SEMG signal processing and with the power of computers, modeling of SEMG is feasible and applicable. There are several benefits of modeling SEMG. First, it provides a better understanding of real life processes and allows the estimation of the internal process characteristics. With the modeling, it is possible to reproduce experimental results by adapting the model's parameters. Moreover, since model-generated data have known properties, these data are well suited and widely applied for algorithm evaluation and performance comparison [44]. Second, modeling SEMG signal also benefits biological and medical education, in which the model is used to test the knowledge and insight acquired by the learner [67].

SEMG models can be grouped into three categories, according to its similarity level to the real process. They are descriptive models, phenomenological models, and structure-based models. A descriptive model can assume certain characteristics in the actual process with limited validity, for example, the proportionality between EMG amplitude and muscle force. A phenomenological model can mimic real phenomena under a wide range of conditions, but is not necessarily coupled to any underlying actual elements evoking the observed outcomes. An example of phenomenological models is an autoregressive model [59]. Instead of giving better understanding of the phenomena behind the EMG signal, the purpose of the above models is to extract certain information from the signal based on a limited number of model variables. A structure-based model describes elements of the real system in an analytical format. 


\subsection{Thesis Objectives}

The thesis objective is to research accuracy and robust automated SEMG onset detection methods. By evaluating the performance of some popular approaches in the literature, we investigate the influence of high pass filter to detection accuracy. We also propose an optimal threshold detection approach to improve detection accuracy of threshold-based methods.

\subsection{Thesis Contributions}

The contributions of this thesis are:

1. Analysis of four threshold-based onset detection approaches: Hodges, Bonato, Lidierth, and Abbink. The performance of these approaches was analyzed with synthetic data and real SEMG signals. Synthetic SEMG are often used to evaluate the performance of various onset detection approaches. Given the specific purpose of onset detection, we chose to use a time-domain approach to generate synthetic data. The comparison analysis benefits the understanding of the characteristics of the SEMG signal.

2. Investigation of the influence of high pass filtering to the onset detection accuracy. Since SEMG signal has unwanted data in the low frequency range, high pass filtering has attracted lots of attention in the SEMG processing area. Recently, research has shown that the high frequency components of the SEMG have a high correlation to muscle force [55], [66]; it is hypothesized that high frequency components would provide a higher degree of accuracy for onset 
detection. However, how to design a high pass filter that improves the detection accuracy for various detection approaches remains a challenging issue due to the variation of the detection system. In this work, we analyzed the effect of high pass filtering using different cutoff frequency and concluded that high pass filter is beneficial to real SEMG signal in general; however, its optimal cutoff frequency depends on the specific approach.

\section{Proposed a mechanism to determine an optimal threshold for threshold-} based onset detectors. Due to the popularity of threshold-based detection approaches, how to decide an optimal threshold is an important topic. Most research focus on the conditioning, the decision rule, and the post-processor, but lack the investigation on the key factor, threshold. In this study, we propose a simple and generally applicable approach to decide optimal threshold for various detection functions and evaluate its performance using both synthetic data and real SEMG signals. The experimental results demonstrate that the proposed algorithm is simple, robust, and well performed. Accuracy of onset detection is improved with this optimal threshold.

\subsection{Thesis Organization}

This thesis is organized in the following manner. Chapter 1 is the introduction. Chapter 2 provides background knowledge on EMG signal generation, signal recording, and onset detection. SEMG signal modeling, signal simulation, and details of data recording are discussed in Chapter 3. In Chapter 4, we will review related work on onset detection and 
evaluate the work using the simulate data and real data. Chapter 5 shows how the added high pass filter in conditioning could affect onset detection accuracy, using the synthetic data and real data. In Chapter 6, we proposed a novel onset detection approach and demonstrated its performance using the experimental results. Chapter 7 concludes this thesis and shows its future work. 


\section{Chapter 2 Background}

Electromyography (EMG) is a technique for evaluating and recording the activation signal of muscles. An instrument called an electromyograph detects the electrical potential generated by muscle cells and these recorded signals are known as EMG signals. In this chapter, we provide background knowledge of basic concepts and mechanism of muscle physiology, as well as the basic electrophysiology of the muscle membrane. We also provide background information regarding the application of onset detection for SEMG.

\subsection{Motor Unit Action Potentials}

EMG signals are essentially made up of superimposed motor unit action potentials (MUAPs) from several motor units (MU), which are the smallest functional blocks of the neuromuscular system.

A MUAP is superimposed single muscle fiber action potentials (APs) from muscle fibers within a given MU. As shown in Figure 2.1, a MU contains a single $\alpha$-motoneuron and a number of muscles fibres innervated by this motor neuron. The interface between the $\alpha$-motorneuron and the muscle fiber is known as the neuromuscular junction or motor endplate. A single MU may occupy a relatively large portion of a cross section of a muscle, which is called the motor unit territory. Muscle fibers of a MU are often mingled with those from other MUs. 


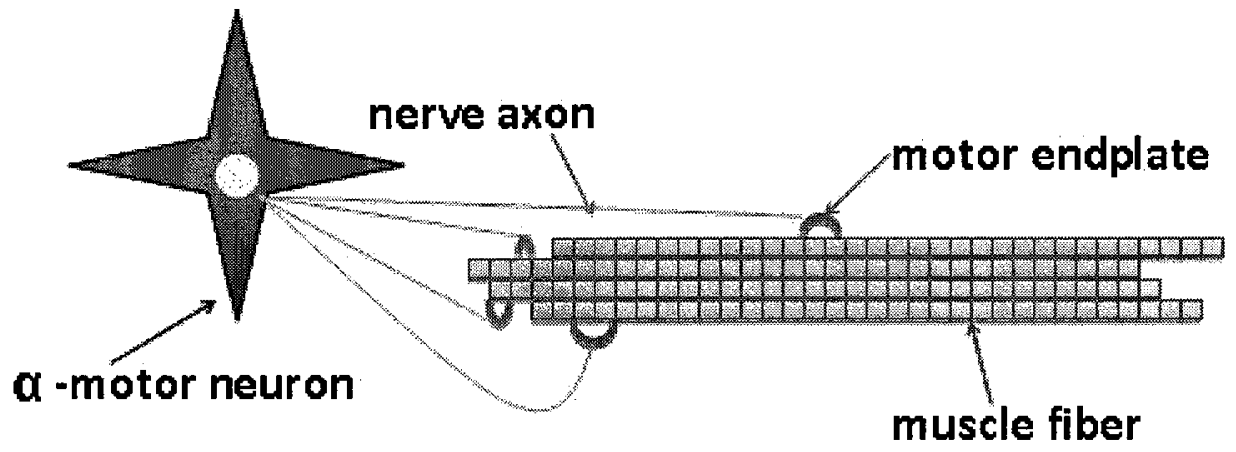

Figure 2.1: A schematic representation of a motor unit, based on [65].

All the muscle fibers of a motor unit are discharged synchronously upon the arrival of a nerve impulse along the axon and through its terminal branches to the motor endplates. The recorded MUAP can be derived from APs from a small number of muscle fibers (e.g., one to three), a moderate number of muscle fibers (e.g., fifteen to twenty), or a larger number of muscle fibers (several hundreds) [44].

\subsection{EMG Signal Generation/Collection}

The EMG signal is comprised of the electrical activity of the active MUs during a contraction. The signal sources are thus located at the depolarized zones of the muscle fibers [44]. Fibers in a MU are grouped together and generate a MUAP. The generation of a MUAP is a process of linearly combining the single fiber action potential signals recorded at the electrode, as shown in Figure 2.2. 


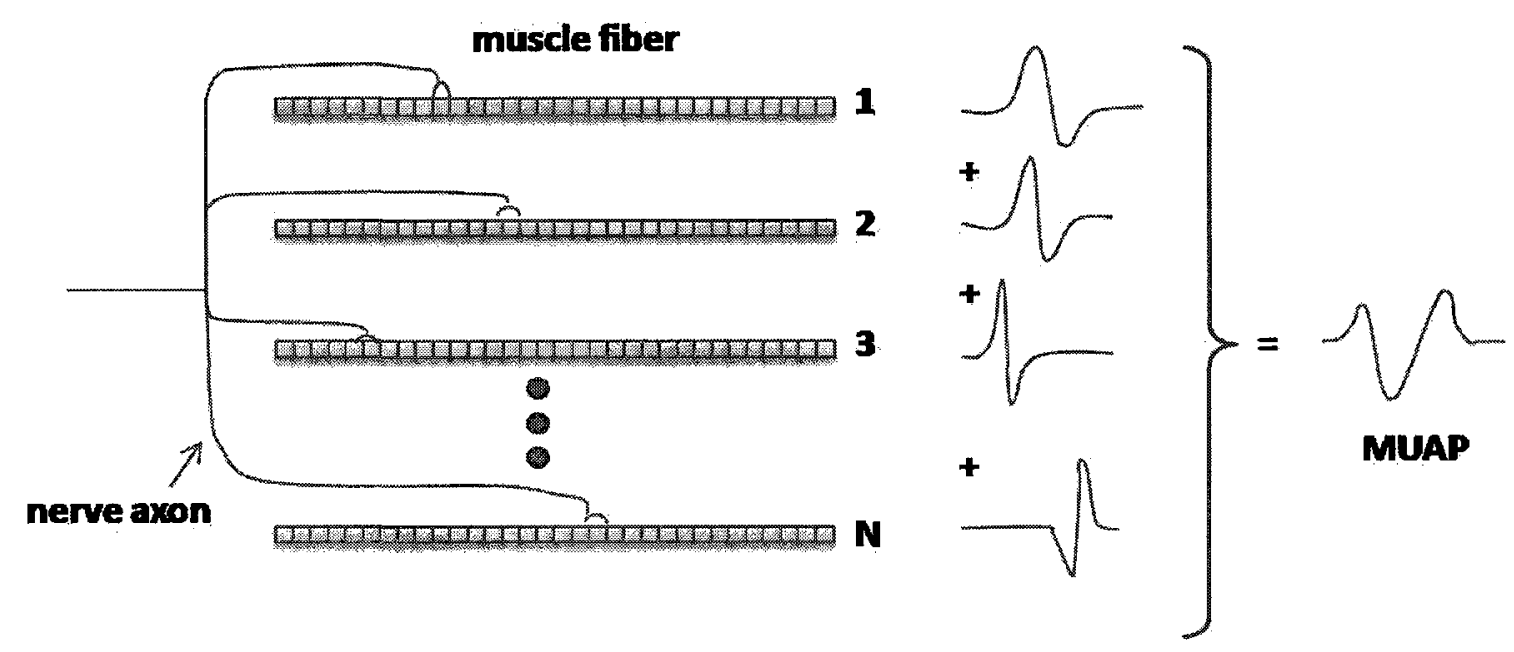

Figure 2.2 A schematic representation of a MUAP [20].

The different MUAPs recorded in a muscle show great variation in shape and other parameters, depending on the electrode position and other detection factors. The detected EMG signal is the superposition of MUAP trains.

There are two ways to record EMG signals: intramuscular recording and surface recording. The former uses invasive needle electrodes, while the latter uses non-invasive surface electrodes. The tissue between electrodes and muscle fibers is a volume conductor. The effect of tissues is relatively small in intramuscular recordings, due to the proximity of the recording electrodes to the sources. For surface recordings, the volume conductor constitutes an important low-pass filtering effect on the EMG signal [44].

Comparing to surface electrodes, needle electrodes are more sensitive to the measurement distance. Thus, needle electrodes can detect mostly activity of the closest muscle fibers, while surface electrodes can sense activity from a larger area within the muscle. In addition, needle EMG signals are suited for the detection of changes in motor 
unit size and its internal structure; while surface EMG (SEMG) data are applied to studies on the various aspects of behaviour (e.g., temporal pattern of activity) of the muscle as a muscle group [44]. In this thesis, we only focus on SEMG.

\subsection{Surface Electromyography}

As stated in Section 2.2, in SEMG, the volume conductor (i.e., biological tissues) has spatial low-pass filtering effect to the SEMG signals; and thus, the SEMG signal presents a smaller bandwidth comparing to the needle EMG signals [2][41].

According to the above description, we can see that SEMG signal recording is influenced by the electrode size, material, and location on the muscle. Other factors include the measurement distance between the source and the detection point, and the spatial low-pass filtering effect of the volume conductor. The influence of the electrode size also has a spatial low-pass filtering effect to SEMG signal, and the electrode's dimensions define the filter shaping [29]. There is also the influence of structural elements of the EMG sources, like the direction of the muscle fibers with respect to the electrode length or width. Due to the variety of electrodes and their arrangement, standardization is very important for SEMG signal and thus Surface Electromyography for Non-invasive Assessment of Muscle (SENIAM; www.seniam.org) was initiated in 1996.

Due to the poor spatial resolution of SEMG, it is difficult to distinguish signal MU activities from sources that are closely placed in the muscle. Based on this property, there are single-channel and multi-channel SEMG signals. The single-channel signals provide 
a global description of the electric potential observed at the recording site, and are often applied to study the interference pattern that results from the simultaneous activation of many MUs; however, the analysis of the single MU activities can be important to research of muscle control and medical diagnosis of neuromuscular disorders. Therefore, multi-channel signals attract more interest recently, due to its capability of extracting single MU activity from the compound signal.

There are two classical techniques in multi-channel recording: 1) linear electrode array and 2) two-dimensional electrodes (e.g., grid). Figure 2.3 shows an example of a linear electrode array using bipolar detection on biceps branchii. Bi-polar electrodes should be placed before or after the innervation zone so that the recordings are consistent for one direction of propagation. A linear electrode array can be used to help locate the innervation zone and choose a bipoloar electrode pair that is away from the innervation zone. 


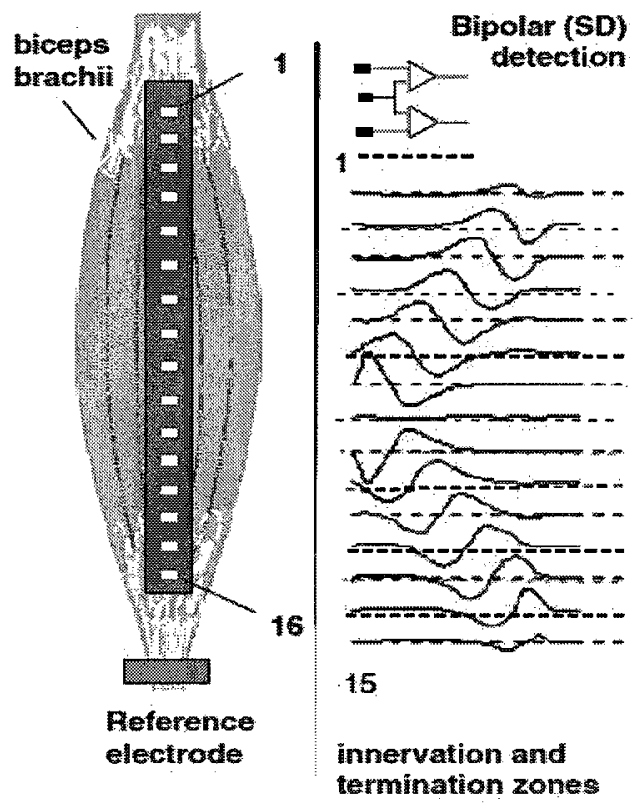

Figure 2.3 Bipolar detection of EMG signal. Source: [44].

Bipolar electrodes provide improved spatial and temporal resolution by effective differentiating the SEMG signal. Grid electrodes permit a more refined filtering in a 2dimensional plane [52], [53].

\subsection{Observations in Electromyography Data Recording}

An EMG signal typically has information in time-domain and frequency-domain, which directly reflects to the amplitude and spectrum of the signal.

The relation between EMG amplitude and force depends on many other factors, such as recording related factors (i.e., electrode location/orientation, the inter-electrode distance, etc.), and the MU related factors (the distribution of conduction velocities of the active MUs, the degree of synchronization of the active MUs, etc.). It is observed that 
both the force and the EMG amplitude are nonlinear to the neural drive in most circumstances; however, this double nonlinearity leads to a close-to-linear relation between EMG and force [44]. Thus, EMG amplitude remains an indicator of muscle activation, which is very useful in the further applications.

The spectrum of EMG signal could be affected by the biological tissue (i.e., volume conductor) or the firing patterns, etc [44]. The firing patterns reflect the motor control of the central nervous system and it often causes the nonconstant spectrum of the point process at low frequency region that is mainly below $30 \mathrm{~Hz}$ [44]. Thus, the influence of the firing patterns of the MUs on the SEMG power spectrum can be neglected in many applications if a high-pass filter is used for SEMG data conditioning.

\subsection{Electromyography Data Modeling}

There are three purposes of modeling SEMG data: better understanding real processes, estimating internal process with limited parameters, and generate synthetic EMG signal to evaluate various algorithms. Accordingly, there are three types of SEMG models: descriptive models, structure-based models, and phenomenological models. The first two models are more on describing the actual recording system; while the third one is focused on simulating the characteristics of the SEMG signals.

We will concentrate on phenomenological models, which are used to generate synthetic data for algorithm evaluation in this thesis. 


\subsubsection{Descriptive Models}

Descriptive models usually have descriptive character with limited validity [44]. A typical example is the model to describe the proportionality between EMG amplitude and muscle force. The model could be a linear shape [4], [71], or a parabolic shape [38], which depends on the recording system (e.g., electrode location/orientation) and the MU/muscle properties (e.g., the location of the MUs within the muscle).

\subsubsection{Structure-based Models}

A structure-based model describes elements of the real system's structure in a mathematically tractable way. The important elements in a recording system or the signal generation mechanism are represented as different models.

In general, these factors in the generation process of the SEMG signal are modeled as multi-dimensional filtering operations in space and time domain [23], and the SEMG signal is a filtered output of the system. For example, due to the relatively large size electrode in SEMG, the action potential (AP) detected is often considered as the average of the potentials of points under the electrode surface. Thus, these point electrodes are modeled as finite impulse response (FIR) filter [18], and the biological tissue under electrodes (i.e., volume conductor) is often modeled as a low-pass filter in spatial domain for SEMG. Thus, the generation of MUAP is modeled as the 2-D convolution of the potential with a spatial filter with impulse response $H_{\text {size }}(x, z)$, in which $x, z$ defines the electrode shape and size. 
In this type of model, SEMG signal is considered as a summation of the MUAP trains, each of which is considered as the convolution of a sequence of Dirac functions (representing the firing instants) with the MUAP wave shape. In addition, a MU firing process is described as consecutive inter pulse intervals (IPIs), which is further modeled as independent random variables following Gaussian distribution [36], [51], Weibull distribution [16], Poisson distribution [9], or gamma distribution [58].

Among different types of structure-based modeling, inverse modeling is emerging as the most practically relevant type, which estimates model parameters from experimental results. Based on the knowledge from inverse modeling, we can simplify the recording process and combine the structure-based model with the phenomenological model for further analysis. For example, we can extract MUAPs with different shapes from real EMG signal, and then generate synthetic ones by changing synchronization levels between MUs and MUAP shapes.

\subsubsection{Phenomenological Models}

Phenomenological models give mathematical representations to detect and quantitatively describe the recorded EMG signal. This type of models is not meant to help better understanding of the phenomena behind the EMG signals; instead, they are often applied to generate synthetic signals for algorithm evaluation [1].

Phenomenological models can estimate EMG amplitude and are used in this thesis for synthetic data generation. Phenomenological models can be grouped in two categories: frequency-domain approaches and time-domain approaches. 


\section{Frequency-Domain approaches}

This type of approaches is often based on the spectrum of the SEMG signal, which is the summation of the spectra of the MUAP trains. The spectrum of the MUAP is modeled as deterministic finite energy signal and that of the point process describing the firing pattern is modeled as random process [1]. With the assumption that the inter-pulse intervals are uncorrelated, the spectrum of a MUAP train is the product of the spectrum of the MUAP and that of the firing pattern.

In a typical model of this type, a transfer function with limited parameters is proposed to model the power spectrum density (PSD) function of real data. Synthetic data is generated by feeding the white Gaussian noise through the shaping filter with this transfer function and then added by another white noise. The synthetic SEMG signal $m_{k}$ is generated by feeding $w_{k}$ through the system shown in Figure 2.4 .

This model produces a measured SEMG $\left(m_{k}\right)$ with statistical properties similar to real EMG [22]. In this model, $w_{k}$ is a zero-mean white process of unit variance, that is widesense-stationary (WSS), and correlation-ergodic (CE). The shaping filter $H_{\text {time }}\left(e^{j \omega}\right)$ represents the spectral shape of the EMG, and could have different types, such as autoregressive moving average (ARMA) or autoregressive (AR) models [43]. The shaping filter $H$ preserves signal variance and $s_{k}$ is the EMG amplitude based on the level of muscular activation. Process $v_{k}$ is another zero-mean, WSS, CE noise process, 
which models the measurement noise and $w_{k}$ and $v_{k}$ are assumed to be uncorrelated with each other.

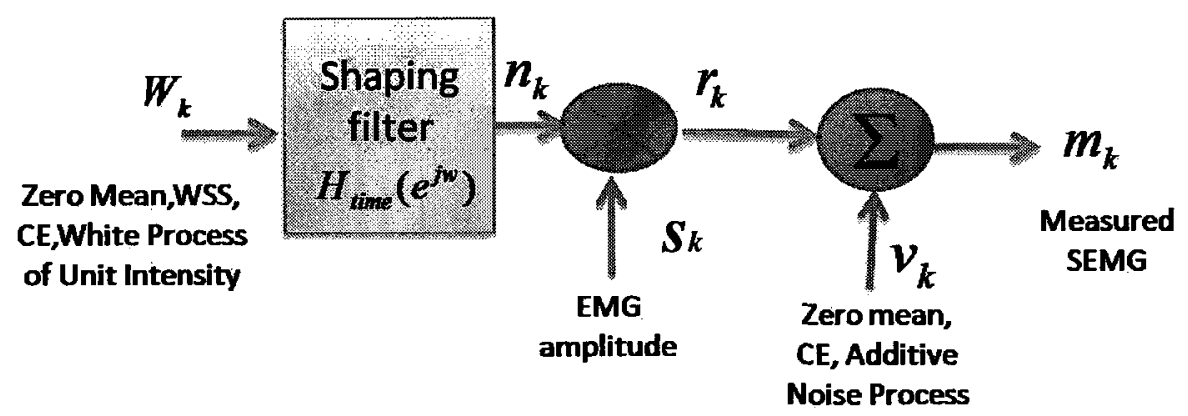

Figure 2.4 Synthetic data generation using frequency-domain approaches, base on [11].

\section{Time-Domain Approaches}

From mathematical perspective, EMG amplitude estimation is to estimate the standard deviation of a colored random process in additive noise. In 1952, Inman et al. [33] implemented a full-wave rectifier followed by a resistor-capacitor low-pass filter, which is considered as the first continuous EMG amplitude estimator. Other estimators include mean absolute value (MAV), i.e., average rectified value (ARV), and root-mean-square (RMS) indicators, with equations:

$$
A R V=\frac{1}{N} \sum_{i=1}^{N}\left|x_{i}\right| \text { and } R M S=\sqrt{\frac{1}{N} \sum_{i=1}^{N} x_{i}^{2}},
$$

where $\mathrm{x}_{\mathrm{i}}$ are the signal samples and $N$ is the number of samples in the epoch considered.

Similar to the spectral estimation, some recent research conducts a standard cascade of sequential processing stages. As shown in Figure 2.5, the process includes noise and 
interference attenuation, whitening, demodulation, smoothing, and relinearization, which is similar to the process of estimating $s_{k}$ from $m_{k}$ in Figure 2.4.

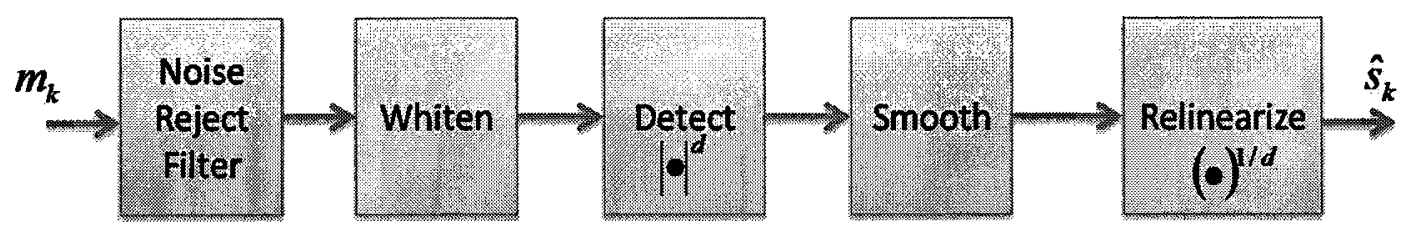

Figure 2.5 Synthetic data generation using time-domain approaches, based on [13].

\subsection{Onset Detection}

The precise detection of the onset of EMG signal is important due to its various applications such as motor control and modern mental chronometry. For instance, the onset could be used as a trigger for recording movement-related brain potentials in EEG signal, such as the synchronization point of the readiness potential [10]. In reaction time (RT) involving externally paced movements, EMG onset has been used to compare stimulus-locked with response-locked brain potentials [25] and fractionate the RT interval into premotor and motor RT to determine whether RT effects are located centrally or peripherally [7], [69].

Onset detection approaches start from completely manually monitoring of the EMG signal, or semi-automatically by letting a computer operate by trial and error. For example, the method proposed by Barrett, Shibasaki, and Neshige [2] relies entirely on visual estimation of the EMG onset, with a preset threshold; the point at which the EMG trace exceeds the threshold is determined by a computer. These methods are time/labor consuming, and the interference of human operator also affects the onset detection 
accuracy. If the threshold level is set too low, the method will be extremely sensitive to small preliminary bursts of EMG activity and hence will underestimate EMG onset. If it is set too high, it will be insensitive to the signal change and overestimate the onset. Moreover, the threshold might be set to different value on a trial base.

Based on the traditional approach, many researchers use certain methodology to select the threshold based on SEMG single properties and to compare it with the rectified SEMG signal to decide the onset value. This kind of methods is popular since it could automatically detect onset value and the human factor is minimized in the preset threshold.

An automatic objective onset detection system for SEMG signal is in great demand, and it is also one of the objectives of this work. 


\section{Chapter 3 Electromyography Data}

In this chapter, we will discuss the SEMG data used in this thesis. These data include synthetic SEMG and real recorded SEMG signal.

\subsection{Synthetic Data Generation}

Performance evaluation of SEMG onset detection algorithms ideally should involve a large amount of SEMG data with well-controlled signal parameters. However, this analysis is difficult or even impossible with real data due to the poor parameter reproducibility of successive SEMG recordings, and the variation among test subjects or even among different trials of one test subjects [44].

Therefore, a popular alternative approach is to use the synthetic SEMG data to assess the accuracy of various detection methods [64]. In this section, we describe the synthetic data used in this thesis.

\subsubsection{Synthetic SEMG Generation}

The approach we used in this section is similar to that in [64], as shown in Figure 3.1. The input is a sequence of random variables with controlled variance. The shaping filter $H(z)$ in the signal model mainly simulated the low pass filtering characteristic of volume conductor and the bandpass characteristic of the electrodes/amplifier combination. 


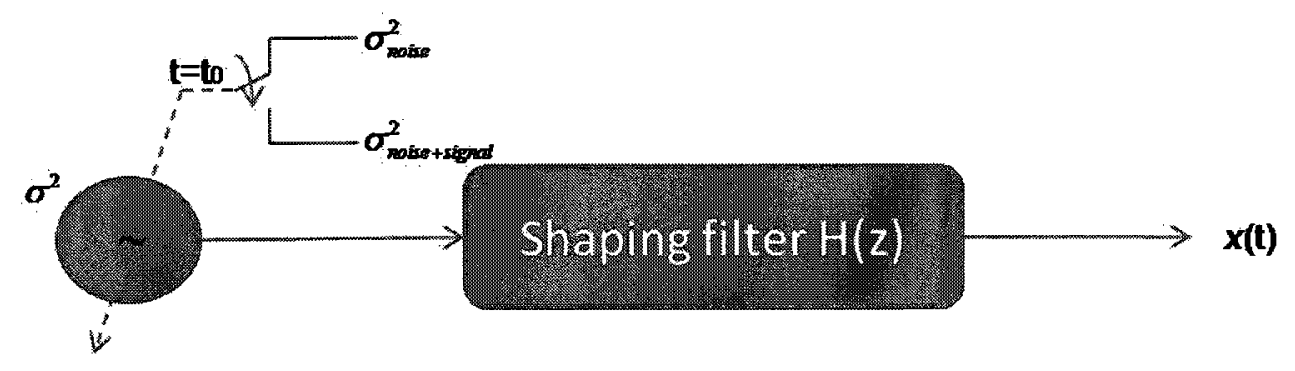

Figure 3.1 The general model to generate synthetic data, based on [64].

The EMG signal sequence $x(t)$ includes a noise period, a transition period, and a period of signal with noise included, as shown in Figure 3.2. The onset time is modeled by rapid change in the variance of $x(t)$. Note that signal-to-noise ratio (SNR) is computed as the following:

$$
S N R=10 \log _{10} \frac{\sigma_{s}^{2}}{\sigma_{n}^{2}}=20 \log _{10} \frac{\sigma_{s}}{\sigma_{n}},
$$

where $\sigma_{s}^{2}, \sigma_{n}^{2}$ are the variance of signal and noise, respectively. In this work, we fix $\sigma_{s}^{2}=1$, so $\sigma_{n}^{2}=1 /\left(10^{0.1 \mathrm{SNR}}\right)$. 


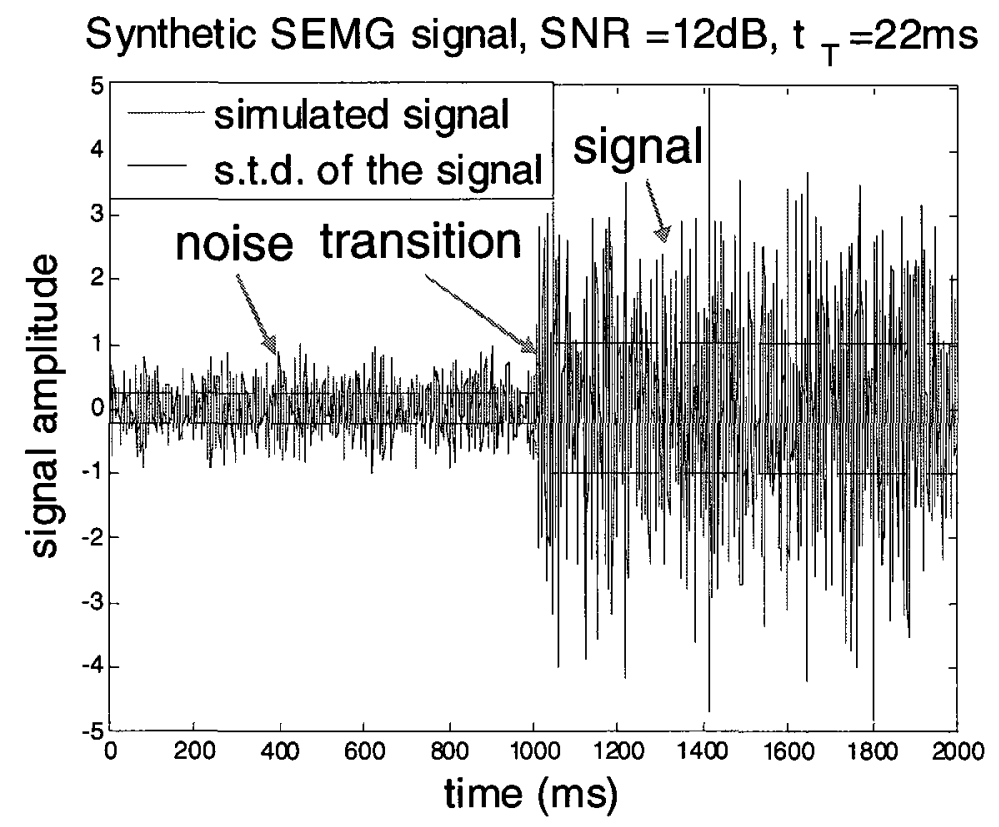

Figure 3.2 Synthetic SEMG signal, with SNR=12dB and transition time $22 \mathrm{~ms}$.

The coefficients of the shaping filter vary with the experimental setup and electrode configuration used for registering the EMG signals, thus these coefficients are not specified in [64]. However, the coefficients can be estimated by using an adaptive whitening filter. In this section, we use a typical set of coefficients provided by G. Staude, the author in [64], which is obtained by fitting a 8th order AR model to EMG data recorded with active surface electrodes at $1 \mathrm{kHz}$ sampling rate. These coefficients are $\{1.0000,-0.3694,0.6645,0.0978,0.2882,0.1569,0.2695,0.0504,0.1706\}$. We use "freqz" function in Matlab to show its frequency response in Figure 3.3. 

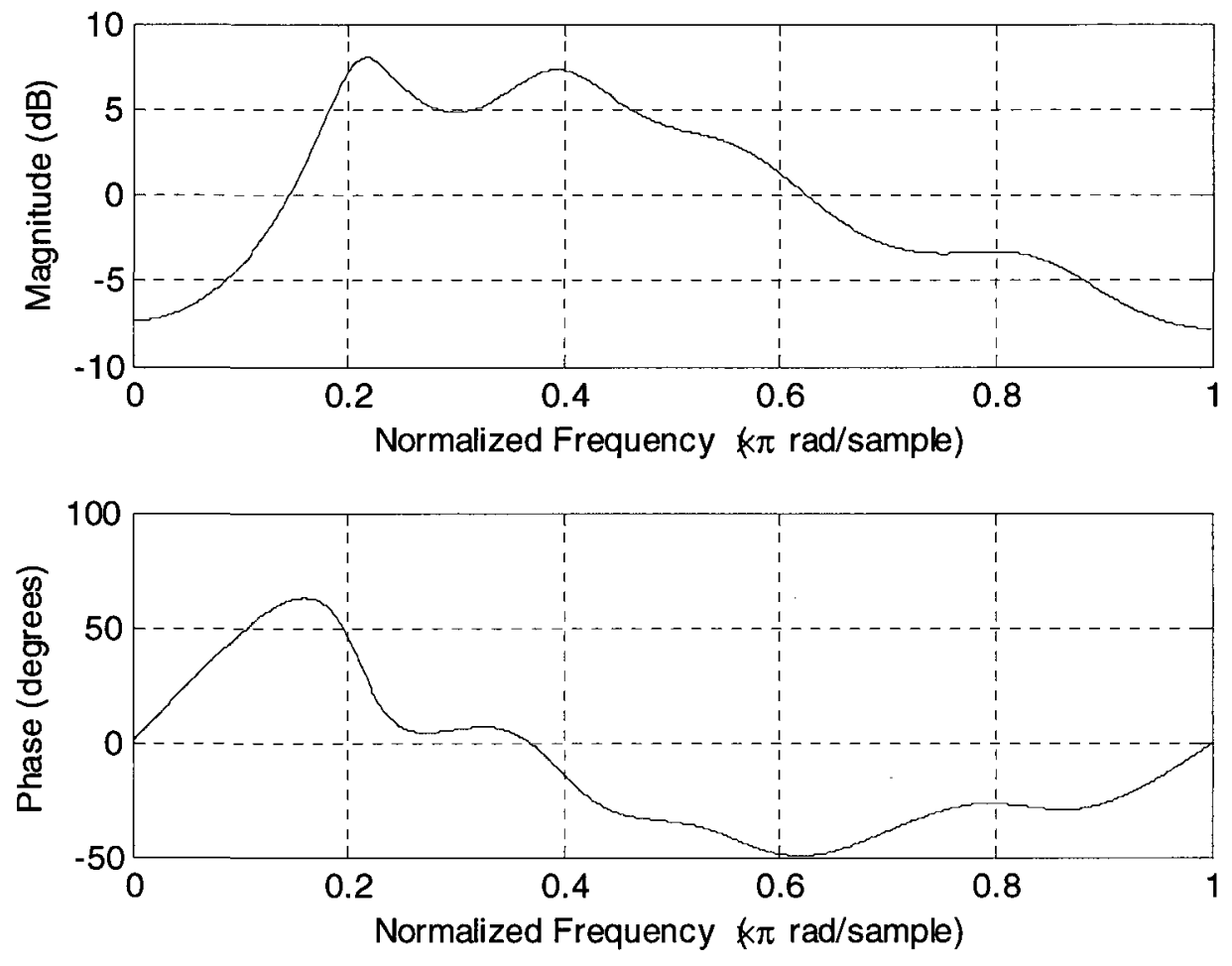

Figure 3.3 Magnitude and phase response of the shaping filter.

We compare the amplitude curve of the signal before and after passing the shaping filter in Figure 3.4. From the figure, we can see that the output from shaping filter has wider amplitude range than the original one, which actually helps the onset detection. 


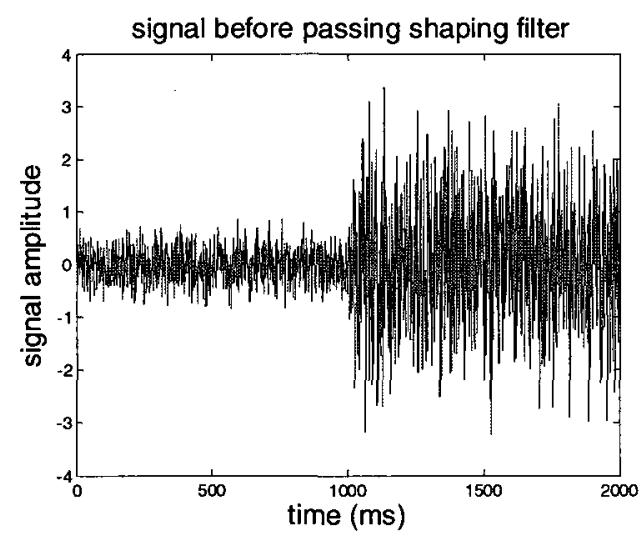

(a)

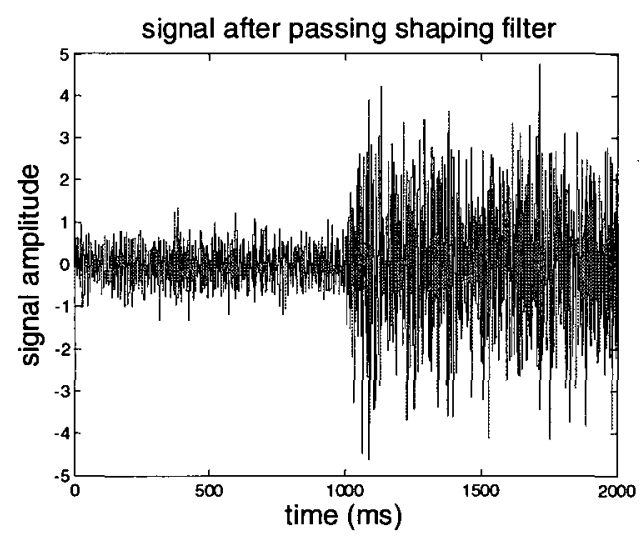

(b)

Figure 3.4 The dataset (a) before and (b) after the shaping filter.

The transition time (or ramp duration) $\tau$ is the time it takes for the signal variance to change from 0 to 1 . It could be either modeled as a fixed number or a uniformly distributed random number in a certain range. It is same with the SNR value, which could be fixed or a uniformly distributed random number belonging to certain range. Thus, to evaluate the sensitivity of onset detection to SNR or transition time, there are four combinations of data sets: 1) random SNR and random transition time, 2) random SNR and constant transition time, 3) constant SNR and constant transition time, and 4) constant SNR and random transition time. These are further described in the following subsections.

In general, these data sets can be generated using the same algorithm shown in Figure 3.5. Before generating $\mathrm{N}$ segments of datasets, we set the length of each segment to $L$, the range of transition period to $\tau_{0}$ and $\tau_{1}$, and the range of SNR value to $S_{0}$ and $S_{1}$. For each segment, the data generation algorithms follow the following procedures: 
For segment $j j$, we have the following procedures:

1. Calculate the transition time as a uniformly distributed number:

$t_{-} T(j j)=\operatorname{round}(\operatorname{rand}(1) *(\tau 1-\tau 0))+\tau 0$;

2. Calculate the sequence $u(t)$ to describe the dynamic change of the whole segment. $u(t)$ is zero for $t<\tau_{0}$ and one for $t>\tau_{1}$, else:

$u(t)=\frac{t-\tau_{0}}{\tau_{1}-\tau_{0}}, \tau_{0} \leq t \leq \tau_{1}$

3. Calculate noise variance according to signal variance (i.e., var_signal $=1$ ) and SNR value, which is a uniformly distributed random number between $\mathrm{s}_{0}$ and $\mathrm{s}_{1}$.

$\operatorname{SNR}(j j)=\operatorname{rand}(1) *\left(s_{1}-s_{0}\right)+s_{0}$;

var_noise $=1 /\left(10^{\wedge}(0.1 * \operatorname{SNR}(j j))\right)$;

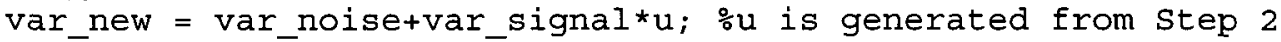

$\sigma$ new = sqrt (var_new); $\%$ calculate the std of the sequence

4. Generate signal with length $L$ and the required standard deviation.

sequence $=\operatorname{randn}(1, \mathrm{~N}) . \sigma^{*}$ new;

5. Send the sequence through the shaping filter, e.g., with coefficients $a$ in [64].

$\operatorname{data}(:, j j)=$ filter $(1, a$, sequence $)$;

Figure 3.5 Procedures to generate synthetic signal.

\subsubsection{Random SNR and Random Transition Time}

In this dataset, the SNR and the transition time are both randomized. The transition period is a uniform random variable between $5 \mathrm{~ms}$ to $30 \mathrm{~ms}$, while SNR is uniformly distributed random variable between $6 \mathrm{~dB}$ and $12 \mathrm{~dB}$.

A total of 2000 segments were generated based on algorithm in Figure 3.5. Each segment is $2000 \mathrm{~ms}$ long with transition period starting at $1000 \mathrm{~ms}$. The histograms of SNR and transition time for the total data sets are shown in Figure 3.6. These data sets are referred as Random SNR and Random Transition Time. 


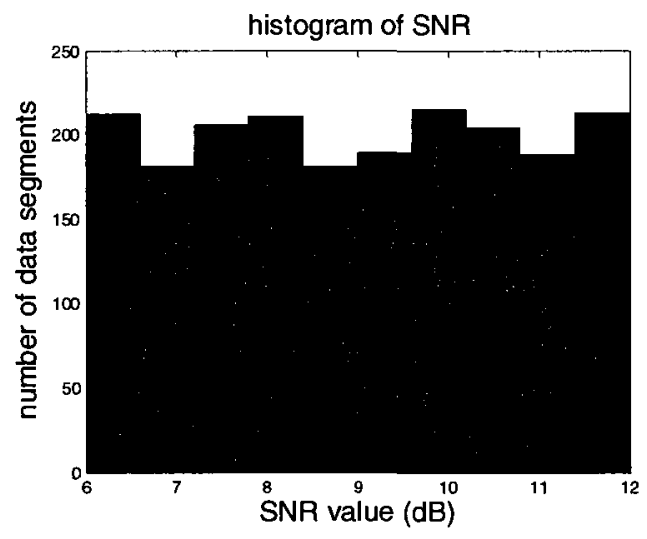

(a)

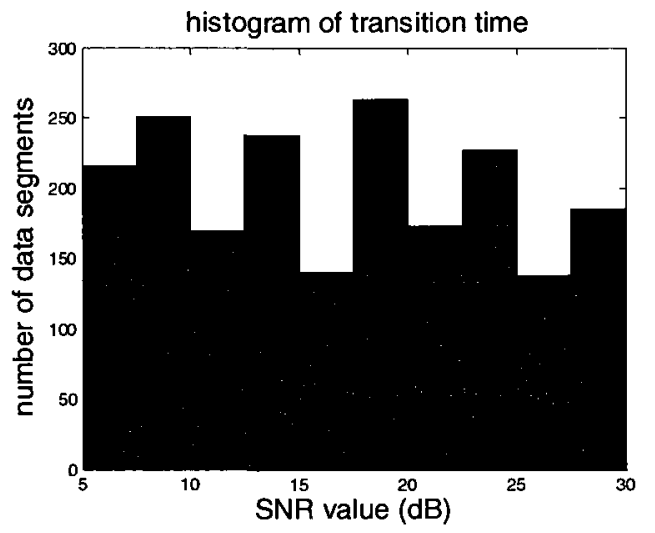

(b)

Figure 3.6 (a) SNR and (b) transition time in Random SNR and Random Transition Time.

Figure 3.7 gives an example of the generated synthetic data, with SNR 7dB and transition time $13 \mathrm{~ms}$.

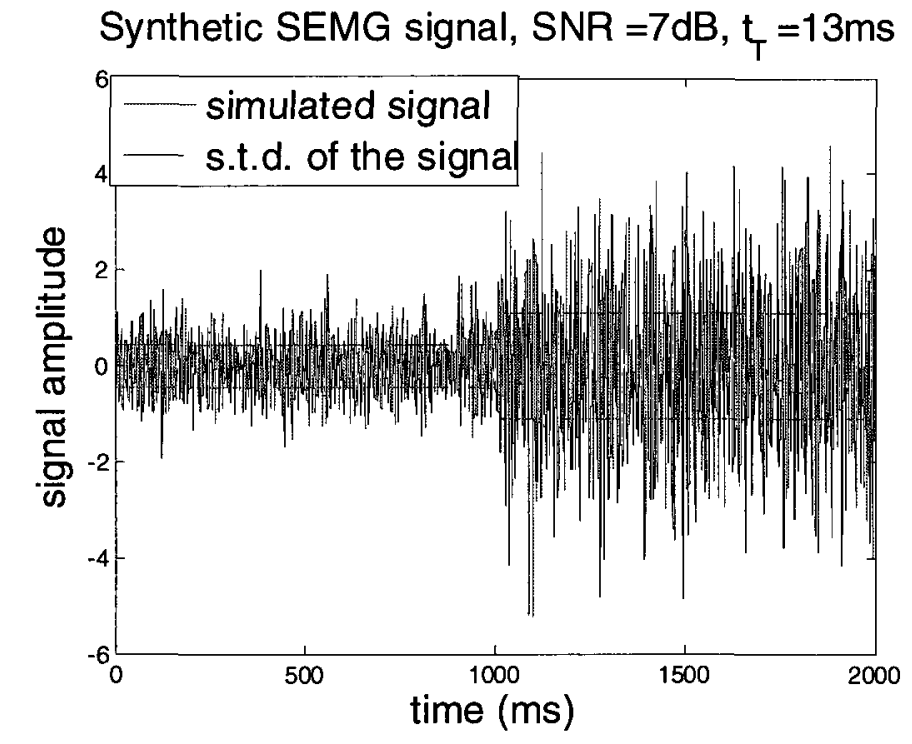

Figure 3.7 Synthetic SEMG signal, with $\mathrm{SNR}=7 \mathrm{~dB}$ and transition time=13ms. 


\subsubsection{Random SNR and Constant Transition Time}

To evaluate the dependence of onset detection on SNR, we generate 700 data segments, with each segment $2000 \mathrm{~ms}$. For SNR at each level between $6 \mathrm{~dB}$ and $12 \mathrm{~dB}$, in increment of $1 \mathrm{~dB}$, we generate 100 segments with random transition time uniformly distributed between $5 \mathrm{~ms}$ to $30 \mathrm{~ms}$.

The algorithm remains the same as shown in Figure 3.5, but SNR is generated in an increasing trend. In total, there are 700 datasets, which are referred as Random SNR and Constant Transition Time. Some examples for different SNR level are shown in Figure 3.8.

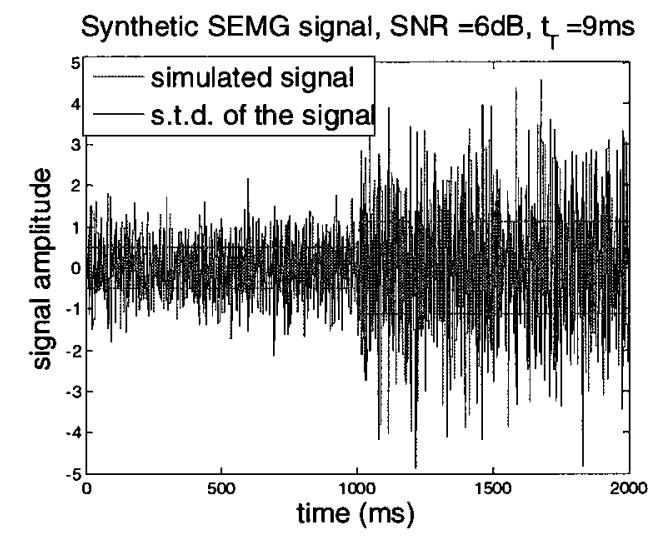

(a)

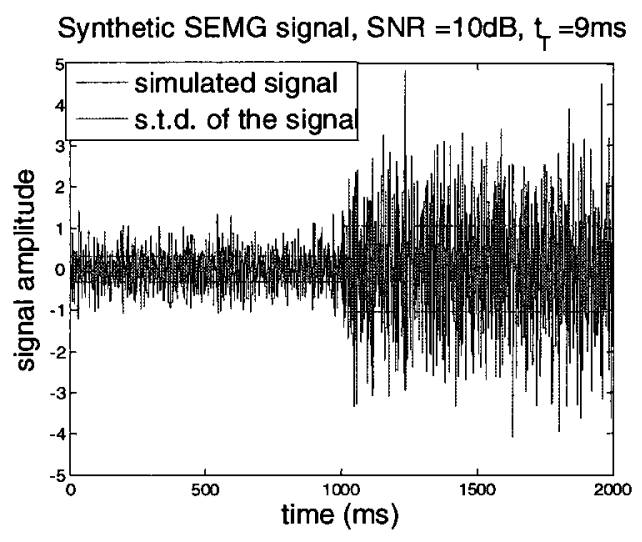

(b)

Figure 3.8 Examples of Random SNR and Constant Transition Time dataset, with (a) SNR = 6dB, (b) SNR = 10dB.

\subsubsection{Constant SNR and Random Transition Time}

To evaluate the dependence of onset detection on transition time, we generate 2600 data segments. The algorithm remains the same as shown in Figure 3.5. For each transition time from $5 \mathrm{~ms}$ to $30 \mathrm{~ms}, 100$ data segments are generated with random SNR, uniformly 
distributed between $6 \mathrm{~dB}$ and $12 \mathrm{~dB}$. In total, there are 2600 datasets, which are referred as Constant SNR and Random Transition Time.

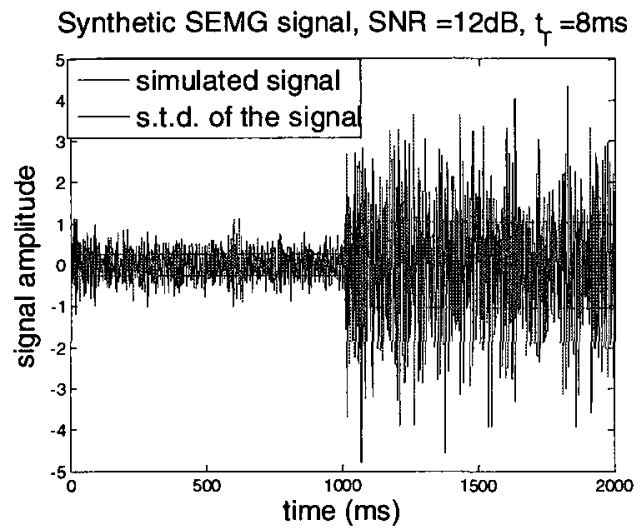

(a)

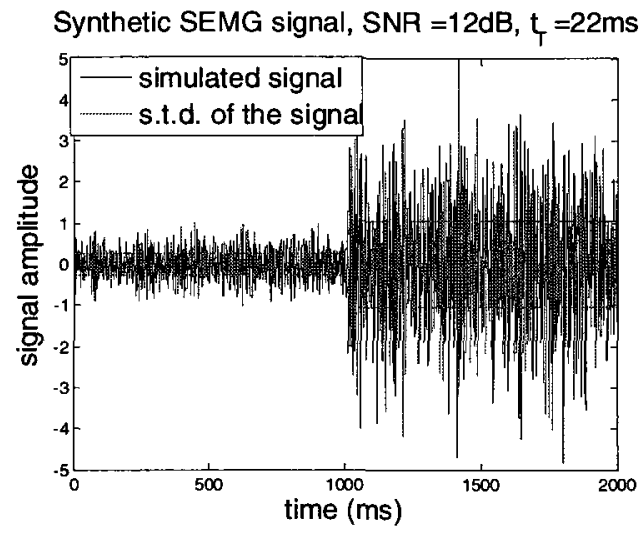

(b)

Figure 3.9 Examples of Constant SNR and Random Transition Time, with transition time

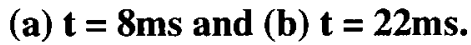

\subsection{Real Data Collection}

The real data used in this study were collected in accordance with guidelines established by the Tri-council policy [68] and the research was approved by the Carleton University Research Ethics Review Board. These data are the same data used for research in myoelectrically controlled prostheses [27].

\subsubsection{Experimental Methods}

During data collection, thirty subjects participated in four sessions on separate days, with each session consisting of six trials. There was one to two days' separation between sessions. Each subject performed seven forearm motions in random order and each motion is performed four times in a whole trial. These motions include hand open, hand 
close, wrist flexion, wrist extension, supination, pronation, and rest. During each motion, the elbow was sustained at 90-degree flexion.

Within each trial, subjects held each limb motion four times three seconds, and thus, the data length is 7 motions $\mathrm{x} 4$ repetitions $\mathrm{x} 3$ seconds $=84$ seconds. A five-second rest period was included at the start and end of each trial to avoid data being cutoff while collecting the data, making each trial 94 seconds in length $(84+5 \times 2)$. These five-second rest periods were removed before data processing. A rest period approximately one minute was provided between each trial.

For each subject in every trial, the forearm myoelectric signals were collected from eight different channels by eight pairs of electrodes placed in the identical location on the right forearm, as shown in Figure 3.10. Electrode placements were marked by permanent markers to ensure the consistency of recording sites throughout all the sessions. The electrode placements were documented by taking digital pictures (one ventral, one dorsal, and two lateral views) during the first session. As shown in the following figures in Figure 3.10, these pictures consist of one ventral, one dorsal, and two lateral views for each subject. 

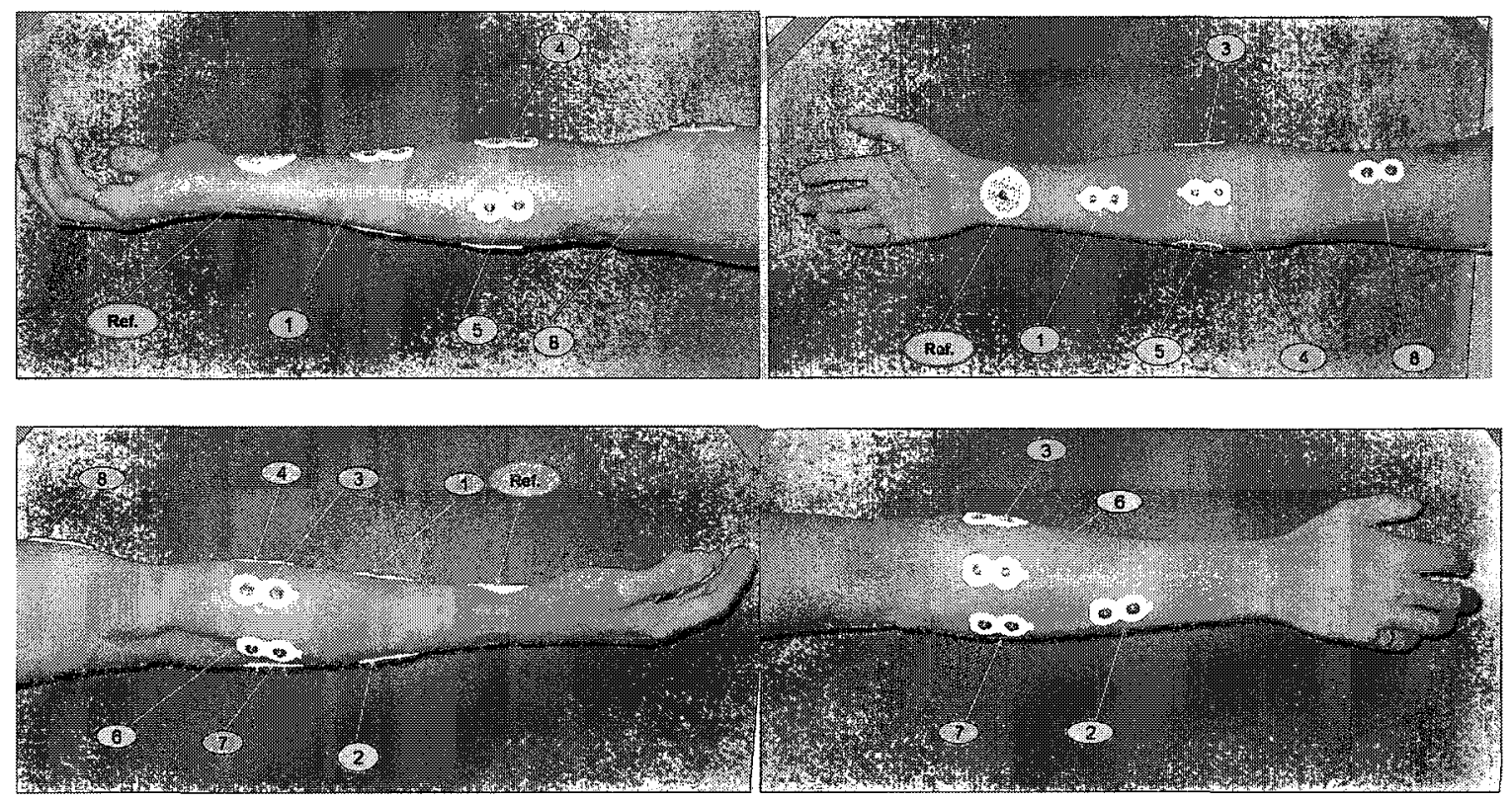

Figune 3.10 Electrode placements from feonn perspectives.

Duo-trode Ag-AgCl electrodes (Myotronics, 6140) were used for the recording. As

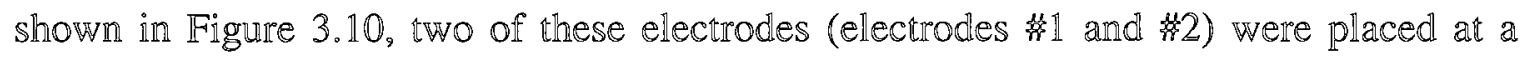
location one third of the forearm length from the wrist to the elbow. Five electrodes (electrodes $\# 3$ to $\#$ ) were placed at a location one third of the forearm length from the elbow to the wrist, with an equal spacing around the forearm [27]. The eighth electrode was placed on the bicep muscle of the upper arm. In addition, an Ag-AgCl Red-Dot electrode (3M, 2237) was placed on the wrist to provide a common ground reference.

\subsubsection{Dar Comditioning}

During the data collection process, noise due to the movement artifacts and instability of the electrode-skin interface often affect data accuracy. Thus, a bandpass filter is often applied to remove noise and retain the "true" EMG signal. 
In our system, SEMG signals were processed through a wide band, high gain AC amplifiers (Model 15A54, Grass Telefactor), with the variable gain set at 1000 and bandwidth set at $1 \mathrm{~Hz}$ to $1 \mathrm{kHz}$ [27].

Moreover, a $60 \mathrm{~Hz}$ notch filter was also applied to reduce power-line interference. Some argue that the notch filter does not help, since it could remove power from a frequency band where EMG shows high-power density and could introduce phase rotation that affects the waveform of the EMG [44]; however, this was not a major concern in our system, since the high power density frequency in our data are not around $60 \mathrm{~Hz}$, and the phase rotation is not important as we are only concerned with the EMG amplitude.

\subsubsection{Data Sampling}

Sampling is an inevitable step in analog-to-digital converting and the Nyquist theorem has to be followed to avoid aliasing. The theorem states that an analog signal that has been sampled can be perfectly reconstructed from the samples if the sampling rate exceeds $2 B$ samples per second, where $B$ is the highest frequency in the original signal [57].

In our system, signals were sampled at $3 \mathrm{kHz}$ using an analog-to-digital converter board (National Instruments, PCI-6071E). The sampled signals were later resampled to 1 $\mathrm{kHz}$ and processed off-line using Matlab. Since the highest frequency to record the SEMG signal usually is $340 \mathrm{~Hz}$ [48], the new sampling frequency is still much higher than the Nyquist frequency. 


\subsubsection{Data Selection and Calibration}

The recorded data were used to create a subset of SEMG onset data from a particular EMG channel and motion. The selected data were collected from channel 5 for motion 3 (wrist flexion), since motion 3 elicits clear muscle activation on channel 5, which corresponds to the flexor carpi ulnaris muscle (FCU) ${ }^{1}$. As shown in the following figure, Figure 3.11(a) shows the flexion action and Figure 3.11(b) shows that we collect signal from Channel 5.

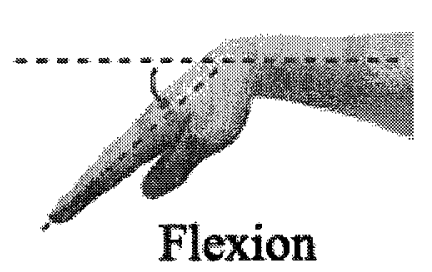

(a)

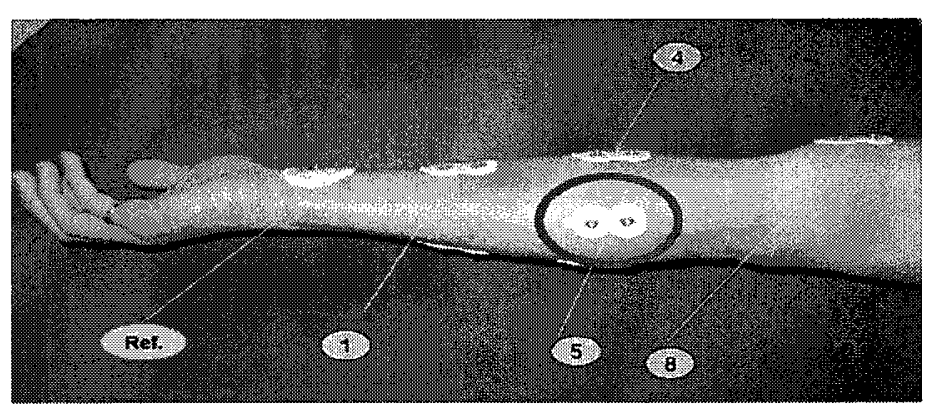

(b)

Figure 3.11 (a) Wrist flexion and (b) Channel 5 to collect data.

Data for all 30 subjects, all 4 sessions, and all 6 trials, were processed and only data from channel 5 from motion 3, with a preceding motion being rest (motion 7) were saved. Each segment of data was $4000 \mathrm{~ms}$ in length (4000 samples, with a sampling rate of 1000 $\mathrm{Hz}$ ), with the onset time approximately in the middle of the segment. The onset for each segment then was determined manually by a researcher with over 10 years of experience with EMG. The real data were examined segment by segment. If the signal of a segment

\footnotetext{
${ }^{1}$ In anatomy, the flexor carpi ulnaris muscle (FCU) is a muscle of the human forearm that acts to flex and adduct the hand [50].
} 
did not have obvious onset time, the segment was discarded. Issues that may have obscured the onset time included excessive noise and signal bursts prior to onset due to pre-contraction twitches.

Each segment had its onset time determined manually six times by a researcher with over 10 years of experience with EMG and all these onset time values are saved as reference for future algorithm evaluation. There were 360 segments in total whose onset was manually determined. The mean of the six manually determined onset times were used as the "gold standard" for the real data. The $4000 \mathrm{~ms}$ length segments were retrieved once again from the original data, such that "gold standard" onset time was located at time around $1000 \mathrm{~ms}$.

Figure 3.12 shows a histogram of the manually determined onset times for all segments (each done six times), which illustrates the variability in manual detection. The standard deviation was found to be $34 \mathrm{~ms}$.

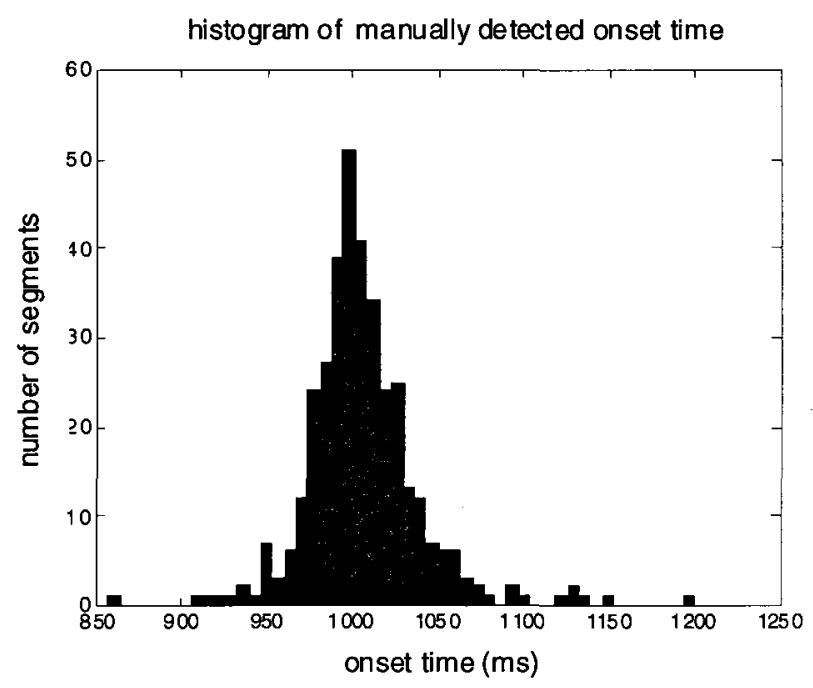

Figure 3.12 Histogram of reference onset time. 


\section{Chapter 4 Onset Detection of SEMG Signals}

Analysis of SEMG signal is very important in the applications of SEMG such as neurological diagnosis [14], [56], neuromuscular and psychomotor research [39], [46], rehabilitation [8], etc. Among the various signal analysis, the detection of the onset time of muscle contraction attracts much attention due to its particular importance in studies of motor control [9] and reaction time (RT) experiments [7]. In this chapter, we give a brief review on SEMG onset detection, and evaluate some popular algorithms.

\subsection{Onset Detection Approaches}

There are many onset detection algorithms introduced in the literature [1], [5], [31], [64], and most detection algorithms consist of up to three basic processing: conditioning; detecting; and post-processing.

\subsubsection{Conditioning/Pre-processing}

In the conditioning stage, a low pass filter is often applied to remove high frequency noise. The raw SEMG signal $\left(x_{k}\right)_{k \geq 1}$ is often rectified and processed by a filter to generate the conditioned data process $\left(y_{k}\right)_{k \geq 1}$. This conditioned data process is a representation of the amplitude or power of the raw SEMG signal. 
In addition, temporal whitening may also be applied to improve amplitude estimate accuracy [11], [12], [13], [15]. There are two ways to design a whitening filter, based on power spectrum function in frequency domain or using linear prediction in time domain.

The process of whitening is to remove the correlation among EMG data samples in $m_{k}$, so that the detection function is able to operate each sample as independent variables. Recall that in Figure 2.4, the shaping filter $H_{s}$ needs to preserve the signal variance since the input white noise has unit variance, and the whitening filter $H_{w}$ is exactly the inverse of the shaping filter $H_{s}$. If we model the SEMG data as in Figure 2.4, we have EMG signal $m_{k}=s_{k} n_{k}+v_{k}$, where $s_{k}$ is the EMG amplitude, $n_{k}$ is the output of shaping filter, and $v_{k}$ is the measurement noise. Since $n_{k}$ is uncorrelated with $v_{k}$, the PSD of $m_{k}$ is:

$$
S_{m m}=s_{k}^{2} \cdot S_{n n}+S_{v v},
$$

where $S_{n n}$ is the output of the shaping filter $H_{s}$ with white noise input $w_{k}$. Thus,

$$
S_{n n}=S_{w w} \cdot\left|H_{s}\right|^{2},
$$

where shaping filter $H_{s}$ is a LTI (linear, time-invariance) system and $w_{k}$ is wide-sensestationary (WSS), and correlation-ergodic (CE), with zero mean and unit variance. Putting Eq. (4-2) into Eq. (4-1), we have

$$
S_{m m}=s_{k}^{2} \cdot S_{w w} \cdot H_{s}{ }^{2}+S_{v v} .
$$

Since input noise $w_{k}$ has white spectrum of unit intensity, $S_{w w}$ is equal to 1 . However, $S_{v v}$ needs to be estimated from real data with 0\% MVC (maximum voluntary contraction), i.e., $s_{k}=0$. Thus, the shaping filter is 


$$
H_{s}=\frac{\sqrt{S_{m m}-S_{v v}}}{s_{k}},
$$

and the whitening filter is:

$$
H_{w}=\frac{s_{k}}{\sqrt{S_{m m}-S_{v v}}},
$$

where $s_{k}$ can be estimated from the amplitude of real EMG data. As shown in Figure 4.1, the shaping filter well described the frequency property of real data, and thus the inverse of the shaping filter, which is the whitening filter, will remove the correlation of original EMG signal and provide almost white noise.

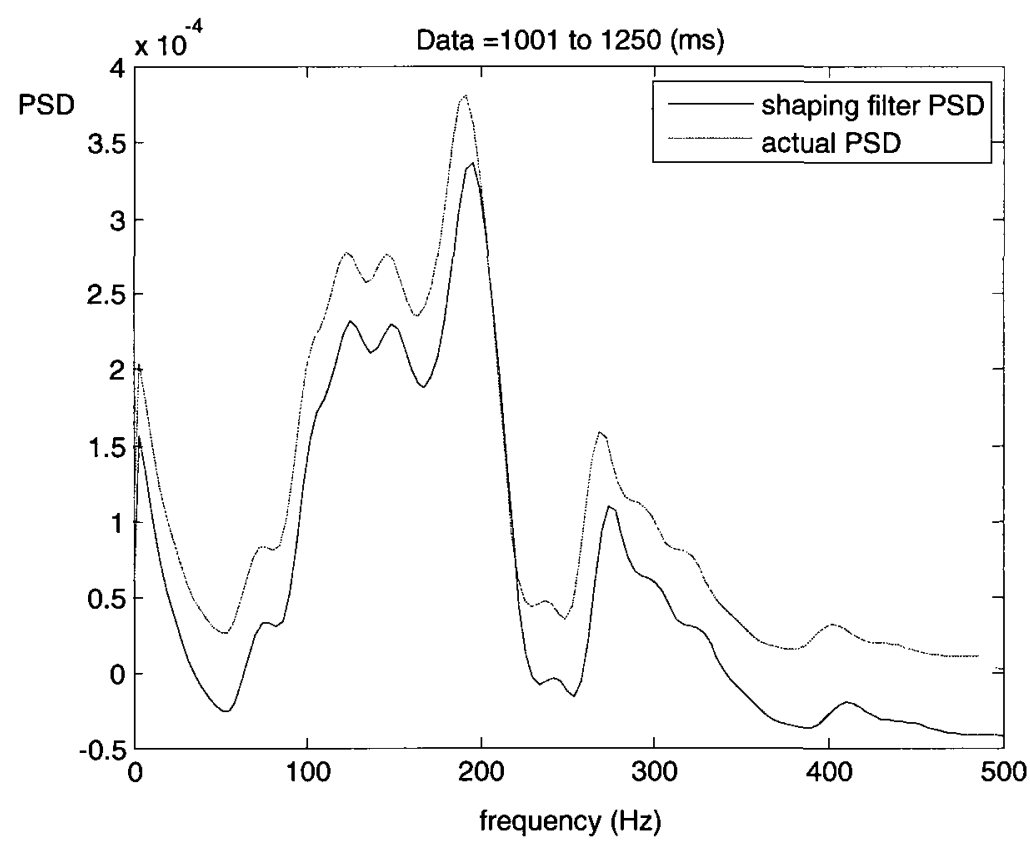

Figure 4.1 The PSD of shaping filter and real EMG signal.

Another approach is to use linear prediction to do whitening. Linear prediction designs a forward linear predictor by minimizing the prediction error in the least squares sense [34]. The estimator computes the estimated signal, and the prediction error, and 
autocorrelation sequence of the prediction error. Since the estimated signal has strong correlation with the original data, the prediction error is approximately white noise sequence with constant power spectrum. As shown in Figure 4.2, filter $H(z)$ functions as predictor and the prediction error $e_{k}=x_{k}-\hat{x}_{k}$ is the desired output of the whitening filter.

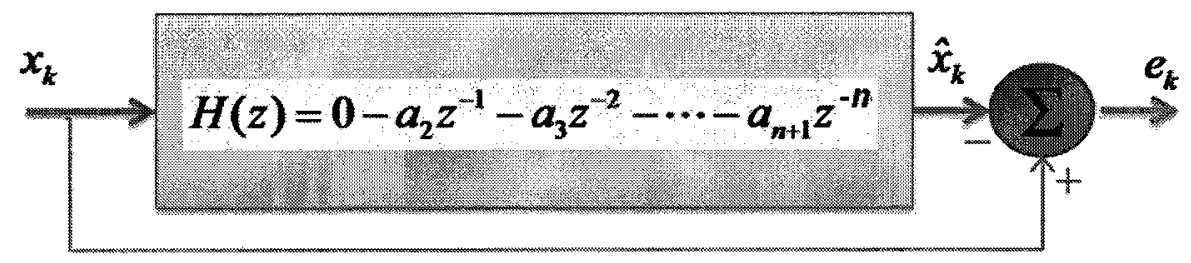

Figure 4.2 The flow of linear prediction.

Temporal whitening removes the predictable components in EMG data that are predominantly in the low frequency range. From this perspective, whitening is similar to high pass filtering, which is much easier to implement since most EMG amplifiers have a built-in high pass filter. Moreover, recent research argue that adaptive whitening, single AR whitening and high pass filtering have little difference in removing the non-stochastic elements of the raw SEMG signal [55]. Nevertheless, we do not verify this claim in this work due to time limit.

\subsubsection{Detecting}

In this stage, a test function is applied to the conditioned signal, i.e., the output from the conditioning.

The test function uses a set of samples to create an alarm time $t_{a}$ that is monitored by the decision rule in order to determine the final onset time. Some detection methods directly use the alarm time $t_{a}$ as an estimate of the onset time and some use $t_{a}$ as a pure 
indicator for the existence of an onset and estimate the onset time through additional post processing. The decision rule is often a stopping rule decided by a threshold, such as $t_{a}=\min \left\{k \geq 1: g\left(y_{1}, y_{2}, \ldots, y_{k}\right) \geq h\right\}$, where $h$ is threshold, $g($.$) is the test function, and$ $\left(y_{k}\right)_{k \geq 1}$ are conditioned data.

\subsubsection{Post-processing}

Note that not all onset detection algorithms use post-processor to estimate onset time.

The post-processor usually starts after alarm time $t_{a}$, and processes the conditioned signal $y_{1}, y_{2}, \ldots, y_{t_{a}+\Delta}$. However, some post-processors process from raw SEMG signal $x_{1}, x_{2}, \ldots, x_{t_{a}+\Delta}$. In addition, most post-processors apply a sliding window starting from alarm time $t_{a}$ to detect onset time. They also assume that the actual onset time happen at least $\Delta$ number of samples after the alarm time $t_{a}$, and thus $t_{a}+\Delta$ represents the earliest possible time for the onset estimate.

\subsection{Algorithms and Implementation}

In this section, we first review some prior work and evaluate their performance. There are two groups of onset detection algorithms: threshold-based and statistical-based. The former is quite popular in this field due to its simplicity and wide applicability, while the latter is applicable only if the signal generating process is (at least partially) known.

With model-based detection concept, statistical-based approaches evaluate the statistical properties of the measured SEMG signal before and after a possible change in model parameters to estimate the onset time $t_{0}$. Since signal generating process is often 
unavailable in real life, we do not implement this type of approaches to the real data and only focus at threshold-based approach in this work.

Threshold-based detection algorithms include single-threshold approaches and double-threshold approaches. Single-threshold approaches determine the onset value based one threshold and double-threshold approaches often add more judgement in the post-processors. Intuitively, double-threshold detectors are superior to single-threshold ones, for two reasons [5]. First, for a fixed value of false-alarm probability, they yield higher detection probability. Second, double-threshold detectors allow the user to adjust the relationship between false alarm and detection probability with a higher degree of freedom than single-threshold ones. However, the increased freedom also causes the instability and difficulty in selecting parameters.

Popular threshold-based detection algorithms include the Hodges' approach [31], Bonato's approach [5], Lidierth's approach [40], and Abbink's approach [1]. The Hodge's approach is single-threshold method and all the other three use doublethresholds.

These threshold-based algorithms can be all considered as finite moving average (FMA) algorithms, in which $H(z)$ is modeled by an all-pole representation (i.e., autoregressive AR filter) of order $p$ and a fixed-size sliding test window is applied. The test function value at time $k$ is the weighted sum of the $W$ signal samples $y_{k-w+1}, y_{k-w+2}, \ldots, y_{k}$ contained within the window. 


\subsubsection{Hodges' Method}

Hodges' method [31] is a representative for FMA methods. It first rectifies and lowpass filters the SEMG signal. A sliding window is applied to the filtered output, which functions as another LPF.

Assuming the $M$ initial samples are noise, the test function $g\left(y_{1}, y_{2}, \ldots, y_{k}\right)$ is obtained by the conditioned data minus the mean of these $M$ samples and dividing by the standard deviation of these $M$ samples. This calculation normalizes the noise to be zeromean and unit variance, which improves the robustness of the detection function, given the assumption that the signal has higher variance than the noise. The alarm time $t_{a}=\min \left\{k \geq W: g_{k} \geq h\right\}$, where $W$ is the sliding window size, $h$ is the threshold, and the onset estimate $\hat{t}_{0}=t_{a}-W+1$. The algorithm is summarized in Figure 4.3. 
1. Rectify and lowpass filter raw SEMG.

$y=\operatorname{abs}(x) ;[b, a]=\operatorname{butter}\left(3,50 /(f s / 2)\right.$, 'low' $\left.^{\prime}\right) ;$

$y=$ filtfilt $(b, a, y) ;$

2. Apply a sliding window of width $W$ on $y_{1}, y_{2}, \ldots, y_{t_{a}+\Delta}$

$\mathrm{a}=1 ; \mathrm{b}=1 / \mathrm{W}^{*} \operatorname{ones}(1, \mathrm{w}) ; \hat{y}_{k}=\operatorname{filter}(\mathrm{b}, \mathrm{a}, \mathrm{y}) ;$

3. Detect function $g_{k}=\left(\hat{y}_{k}-\hat{\mu}_{0}\right) / \hat{\sigma}_{0}$, where $\hat{y}_{k}=\sum_{i=k-W+1}^{k} y_{k} / W, y_{k}$ is the conditioned data, with $\hat{\mu}_{0}, \hat{\sigma}_{0}$ as the mean and standard deviation of the first $M$ sample of data $y_{k}$.

$\hat{\mu}_{0}=\operatorname{mean}(\mathrm{y}(1: \mathrm{M})) ; \hat{\sigma}_{0}=\operatorname{std}(\mathrm{y}(1: \mathrm{M})) ; g_{\mathrm{k}}=1 / \hat{\sigma}_{0} *\left(\hat{y}_{k}-\hat{\mu}_{0}\right) ;$

4. The alarm time $t_{a}=\min \left\{k \geq W: g_{k} \geq h\right\}$

ind $=\mathrm{find}\left(\mathrm{g}_{\mathrm{k}}>=\mathrm{h}\right) ; t_{a}=\operatorname{ind}(1)$;

The onset estimate is $\hat{t}_{0}=t_{a}-W+1$

\section{Figure 4.3 Implementation of Hodges' approach.}

\subsubsection{Bonato Method}

Different from Hodges' method, Bonato method [5] is a double-threshold detector and it uses a whitening filter in conditioning to guarantee the independence of successive samples.

Besides the number of threshold, Bonato also differs from Hodges in its conditioning algorithm. Bonato method uses whitening filter to remove the correlation among variable, so that each variable could be considered irrelevant to its neighbors. An adaptive whitening filter is used for conditioning in this approach. While the design of adaptive whitening filter could be complex and the influence of this filter to the detection accuracy 
could be significant, there is no detailed description of obtaining filter parameters in its original paper. Therefore, we design the whitening filter based on our knowledge.

We use linear prediction approach to do whitening. Due to the possible non-stationary in EMG amplitude [12], we apply a non-overlap window to do whitening. We set the window width to $30 \mathrm{~ms}$, since it is generally accepted that muscle activation shorter than $30 \mathrm{~ms}$ has no effect in controlling the joint motion during gait [6]. Clancy and Hogan use a fourth-order whitening filter in [11] and thus we choose the order of the linear predictor to be four. The pseudo code of the whitening filtering is shown in Figure 4.4.

1. Given raw data $x$. Set window width $\mathrm{ww}=30 \mathrm{~ms}$ and the total number of window is:

Win_num $=$ floor (length $(x) /$ ww);

2. For each window, apply a linear predictor to the raw SEMG.

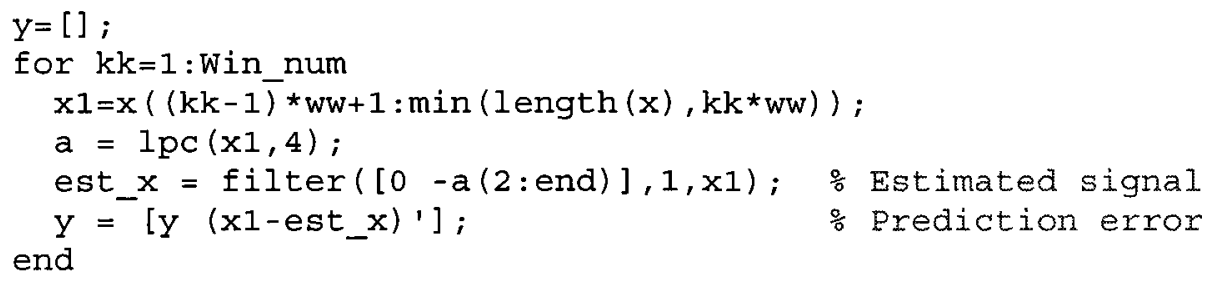

3. Then $y$ is the whitened data.

Figure 4.4 Conditioning stage in Bonato method.

The detection function is the sum of neighboring values of the filtered data divided by the variance of the first $M$ samples, which could be assumed as noise. Thus, the division operation normalizes the noise variance and improves the robustness of the detection function. 
To calculate detection function value, an average of two neighboring conditioned samples is computed. The test function is $g_{k}=\frac{1}{\hat{\sigma}_{0}^{2}}\left(y_{k-1}^{2}+y_{k}^{2}\right)$, where $\hat{\sigma}_{0}$ is the standard deviation of the first $M$ sample of the conditioned data $y_{k}$. The decision rule only checks the $g_{k}$ at odd value of $k$ and the alarm time $t_{a}=\min \left\{k=1,3, \ldots: g_{k} \geq h\right\}$.

This approach uses a relatively complicated post-processor to estimate onset value, which is described in Figure 4.5. Essentially the onset time is found when the SEMG moves from an inactive state to an active state, which lasts for at least $T_{l}$ samples. An active state is defined as having $n$ out of the last $m$ successive samples exceeding the threshold $h$, which could remove the outliers in the samples and reduce the false alarm rate. 
For $y_{1}, y_{2}, \ldots, y_{t_{a}+\Delta}$, a state is claimed to be active only if $n$ out of $m$ successive samples exceed threshold $h$. sum $=0$;

$$
\begin{aligned}
& \text { for } i i=1: t_{a}+\Delta \text {, } \\
& \operatorname{count}(i i)=0 ; \text { state }(i i)=0 ; \\
& \text { for } j j=i i: \min \left(t_{a}+\Delta, i i+m-1\right) \text {, } \\
& \text { if } g_{k}(j j) \geq h, \operatorname{count}(i i)++ \text {; end } \\
& \text { if count }(i i) \geq n \text {; break; end } \\
& \text { end } \\
& \text { if } \operatorname{count}(i i) \geq n \text {, state }(i i)=1 \text {; end } \% \text { active state } \\
& \% \text { Such an active state must last for } T_{1} \text { samples. } \\
& \text { if } \operatorname{state}(i i)=1 \text {, sum }++ \text {; } \\
& \text { else } s u m=0 \text {; end } \\
& \text { if } \operatorname{sum} \geq T_{1}, t_{0}=i i-T_{1}+1 \text {; break; end }
\end{aligned}
$$

\section{Figure 4.5 Detection \& post-processing of Bonato method.}

As discussed earlier, double-threshold approaches have lots of implementation flexibility, which also introduce vulnerability. For example, the parameters and the order of the adaptive whitening filter need to carefully selected, double thresholds ( $h$ and $\mathrm{T}_{1}$ ) are used in post-processor, and there are other undefined parameters such as $n$ and $m$. The selection of these parameters will definitely affect the performance of this method.

\subsubsection{Lidierth Method}

We first get the knowledge of this approach from [64] (as its citation [40]); however, it is interesting to see that the description of this approach in [64] does not follow the 
procedures written in [40]. Here, we still follow the procedures in [64] since the description in [40] is only a simplified version.

Lidierth method can be considered as a combination of Hodges' and Bonato's method. In this approach [40], the conditioning system is a full-wave rectification, while its test function and decision rule are identical to those of Hodges approach and postprocessing similar to Bonato's approach, with $n=1$. It is supposed to have better performance than Hodges' method, since its post-processor add its robustness and reduces the false detection rate. We do not show the pseudo code here since it is captured already in Figure 4.3 and Figure 4.5.

\subsubsection{Abbink Method}

This approach [1] uses similar conditioning, test function, and decision rule as in Hodges approach, with different cutoff frequency and window length $W$ settings. It also uses post-processor, which includes an extra conditioning system (i.e., a Butterworth lowpass filter with $30 \mathrm{~Hz}$ cutoff frequency). Similar to Lidierth method, Abbink method add an extra postprocessor to Hodges' method. However, its post-processor is different from Bonato's.

During the implementation, we found that the original post-processor could be modified to improve the overall performance. For example, the post-processor of Abbink algorithm takes the alarm time, i.e., the first time that detection function is above the threshold, as its window starting time. On the one hand, the accuracy of the alarm time heavily depends on the preset threshold, which could vary from case to case. On the other 
hand, an inaccurate alarm time could introduce extra errors. Thus, we apply a window around each possible alarm time and select the best candidate, which dramatically improve the detection accuracy. Figure 4.6 shows the pseudo code of post-processor.

1. For $y_{1}, y_{2}, \ldots, y_{t_{a}+\Delta}$, generate $y_{1}^{\prime}, y_{2}^{\prime}, \ldots, y_{t_{a}+\Delta}^{\prime}$ using a Butterworth lowpass filter with $30 \mathrm{~Hz}$ cutoff frequency.

2. Estimate mean $\hat{\mu}_{0}$ and standard deviation $\hat{\sigma}_{0}$ from $y_{1}^{\prime}, y_{2}^{\prime}, \ldots, y_{M}^{\prime}$

3. Starting from the first alarm time to the last alarm time,

for $i i=t_{a}(1): t_{a}($ end $)+\Delta$,

$$
\operatorname{count}_{l}(i i)=0 ; \operatorname{count}_{h}(i i)=0 \text {; }
$$

- In the $N$ samples before candidate $i i$, count the number of normalized amplitudes $\left(\hat{y}-\hat{\mu}_{0}\right) / \hat{\sigma}_{0}$ that are smaller than threshold $h_{2}$ for $j j=i i-N+1: i i$,

$$
\text { if } \frac{\hat{y}_{i j}-\hat{\mu}_{0}}{\hat{\sigma}_{0}}<h_{2}, \text { count }_{l}(i i)++ \text {; end }
$$

- In the $N$ samples after candidate $i i$, count the number of normalized amplitudes $\left(\hat{y}-\hat{\mu}_{0}\right) / \hat{\sigma}_{0}$ that are larger than threshold $h_{2}$ for $j j=i i+1: i i+N$, if $\frac{\hat{y}_{j j}-\hat{\mu}_{0}}{\hat{\sigma}_{0}}>h_{2}$, count $_{h}(i i)++$; end

- Sum count $_{l}(i i)$ and count $_{h}(i i)$ and find the onset time estimate $t_{0}$ if $\left(\right.$ count $_{l}(i i)+$ count $\left._{h}(i i)\right)$ is $\max , t_{0}=i i$; end

end

Figure 4.6 Detection \& post-processing for Abbink. 


\subsection{Algorithm Evaluation on Synthetic data}

\subsubsection{Parameter Settings in Approaches}

All these approaches use $a b s$ operator to conduct full-wave rectification in the conditioning system. Other filtering and parameter settings are as the following:

- Hodges approach: sixth-order digital Butterworth low-pass filter with $50 \mathrm{~Hz}$ cutoff frequency for conditioning; set $h$ to $2.5, W$ to 50 , and $M$ to 200 .

- Bonato approach: Use a linear prediction filter of order 10 to do pre-whitening, set $h$ to $7.74, n$ to $1, m$ to $5, T_{1}$ to 20 , and $M$ to 200 .

- Lidierth approach: Set $T_{I}$ to $90, m$ to $15, h$ to 3 , and $M$ to 200 .

- Abbink approach: Use a sixth-order digital Butterworth low-pass filter with $3 \mathrm{~Hz}$ cutoff frequency for conditioning; set $h$ to $3, h_{2}$ to $3, W$ to $1, N$ to 200 , and $M$ to 200.

\subsubsection{Analysis Evaluation}

An objective error measurement of the onset detection is often conducted by comparing the automatically detected onsets to the known onset time, which is preset in synthetic data and visually inspected in real signal. In synthetic data, the accuracy evaluation is relatively easy since the onset time is controllable.

The percentage of detection rate is a good criterion to evaluate the reliability of onset detection methods. It is the ratio of the data segments that have onset successfully detected and the total number of data segments. The higher the percentage of detection 
rate is, the more reliable the detection method is. Failure in the detection of an onset can be due to a number of reasons but an inappropriately high threshold is typical. Other evaluation metrics described in the following paragraphs only apply to the successfully detected onset time after removing those unable to be detected.

We use several popular metrics to evaluate the accuracy of approaches. One is the accuracy function $P_{\alpha}=P\left(\left|\hat{t}_{0}-t_{0}\right| \leq \alpha\right)$, where $\alpha$ is the tolerance of estimate error, $\hat{t}_{0}$ and $t_{0}$ are the estimate and actual onset time value [64]. $P_{\alpha}$ denotes the percentage of estimation error that is smaller than or equal to the tolerance $\alpha$.

Another accuracy metric is the histogram of the detection error $\hat{t}_{0}-t_{0}$ (not absolute value), which shows the asymmetrical property and spread range of the error. An additional accuracy metric is the mean and standard deviation of the detection error, which can be considered as the information extracted from the PDF of the error.

More evaluation metrics include the mean, the standard deviation, and the skewness ${ }^{2}$ of the detection error in these four approaches. Among them, the standard deviation is the most important metric. The mean error is not particularly important. The mean error represents a bias and can be removed easily by accounting for the offset. Skewness is a measure of the asymmetry of the data around the sample mean. The skewness of a distribution is calculated as the following equation:

\footnotetext{
${ }^{2}$ If skewness is negative, the data are spread out more to the left of the mean than to the right. If skewness is positive, the data are spread out more to the right [57].
} 


$$
y=\frac{E(x-\mu)^{3}}{\sigma^{3}},
$$

where $\mu$ and $\sigma$ are the mean and standard deviation of $x$, and $E($.$) is the expectation$ function. Thus, skewness is zero for any perfectly symmetric distribution such as normal distribution.

\subsubsection{Evaluation on Synthetic Data}

Synthetic data generated from phenomenological model is often used as "golden standard" to assess the accuracy of various detection methods [64], since synthetic data are more controllable and more reliable in certain sense.

We use the Random SNR and Random Transition Time that are generated in Section 3.1.2 that includes 2000 data segments and the onset errors are the average of detection error of these segments. In Figure 4.7, we compare the accuracy functions and normalized histogram for each method. As shown in Figure 4.7 (a), x-axis stands for the value of onset detection error, and y-axis stands for the value of $P_{\alpha}$, the percentage of responses with the estimation error smaller than or equal to the corresponding error tolerance on x-axis. The closer and faster the $P_{\alpha}$ curve converges to 1 , the smaller deviation and smaller detection error are. In Figure 4.7 (b), we can see that the detection error is roughly centered at zero with slightly asymmetry, which will be described using skewness in the following analysis. 


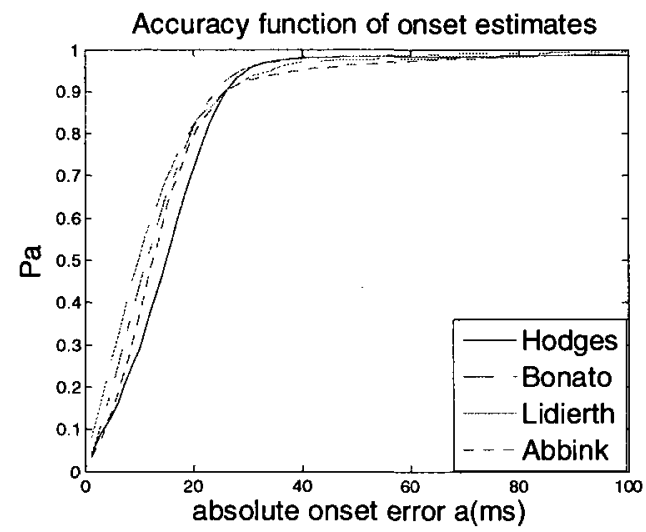

(a)

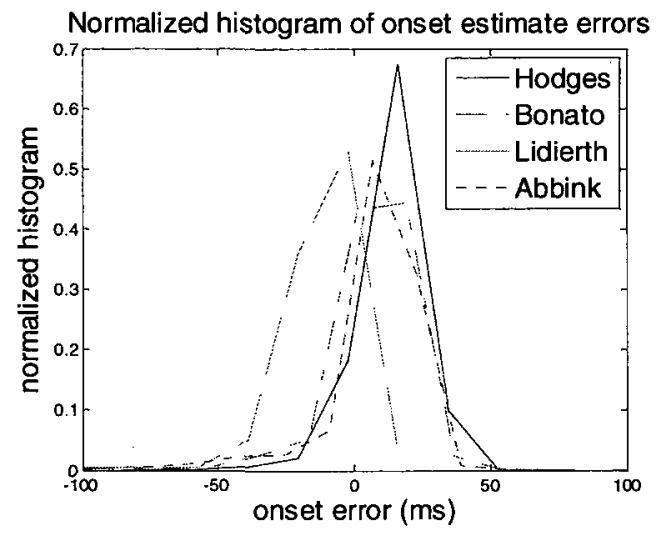

(b)

Figure 4.7 (a) Accuracy function and (b) normalized histograms of all approaches on synthetic data.

Table 4.1 shows the mean, standard deviation, and skewness of the detection error of these four approaches. Negative skewness means that the detected onset time is spread out more to the left of the mean than to the right; otherwise, the data are spread out more to the right. In addition, Table 4.1 shows the detection rate of each approach and the value of accuracy function $P_{\alpha}$, with tolerance error $\alpha=100 \mathrm{~ms}$.

Table 4.1: More comparison results of detection error for four approaches on synthetic data.

\begin{tabular}{ccccc}
\hline Approaches & Hodges & Bonato & Lidierth & Abbink \\
\hline \hline Mean error(ms) & 20 & 9 & -14 & 4 \\
S.T.D. error (ms) & 62 & 30 & 28 & 41 \\
Skewness (ms) & 6.08 & 4.62 & 4.91 & 4.95 \\
Detection rate (\%) & $100 \%$ & $100 \%$ & $100 \%$ & $98.9 \%$ \\
$\mathrm{P}_{100}$ & 0.9860 & 0.9950 & 0.9890 & 0.9894 \\
\hline
\end{tabular}


Comparing the results in [64] to Figure 4.7 in this work, we can see that our implementation has better performance than the original ones. As we stated earlier, certain process has been changed in our implementation. For example, if we use the original post-processor in [64] for Abbink, we have its accuracy function curve together with others in Figure 4.8 and its degradation in Figure 4.8 comparing to it in Figure 4.7 is quite obvious.

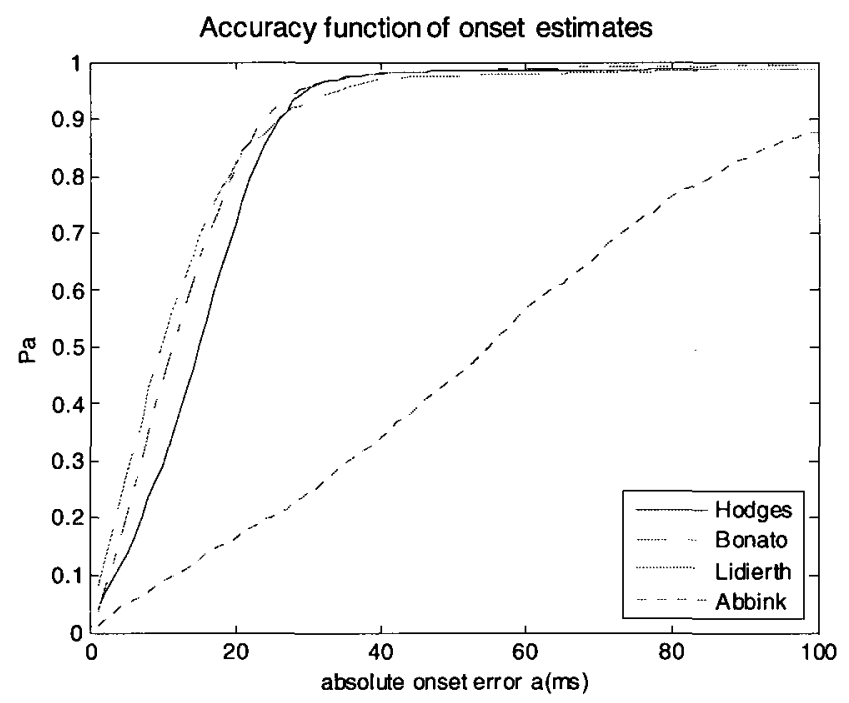

Figure 4.8 Accuracy function of four approaches using the original processes.

Another factor that influences the performance is the parameter setting. In [64], $T_{1}$ in Bonato method was set to $50 \mathrm{~ms}$; however, we notice that since the whitening filter window is only $30 \mathrm{~ms}$, there is not point of setting threshold of active state higher than $30 \mathrm{~ms}$ and an inappropriate setting of parameter could impact the performance. As shown in Figure 4.9, with $T_{1}=50 \mathrm{~ms}$, the accuracy function of Bonato method increases very slowly and $\mathrm{P}_{100}$ is only 0.9440 instead of 0.9960 when $T_{l}$ was set to $20 \mathrm{~ms}$. 


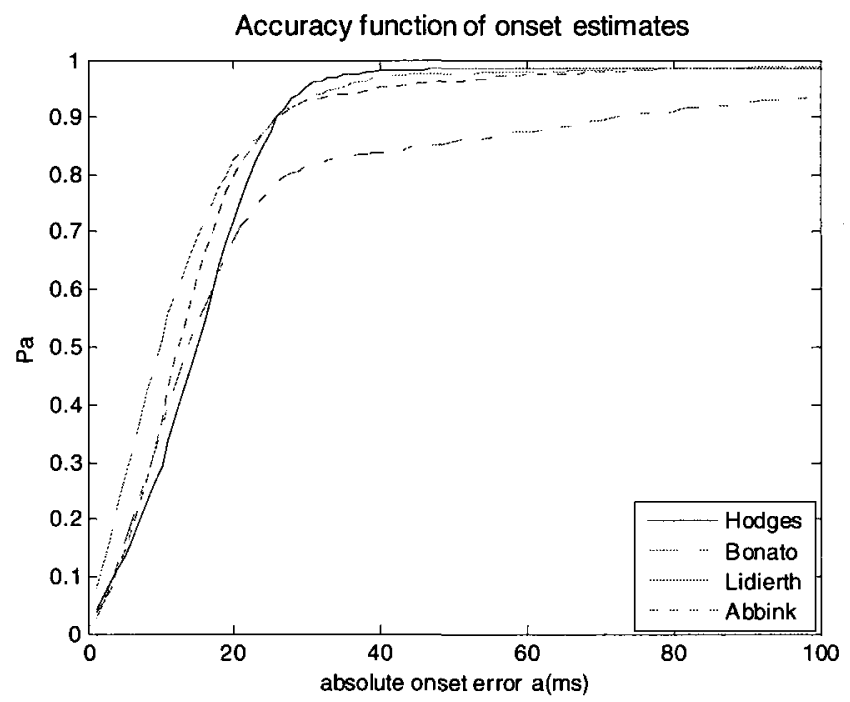

Figure 4.9 Accuracy function of four approaches using original parameter settings.

We also evaluate the dependence of mean and standard deviation of the onset error on SNR using Random SNR and Constant Transition Time generated in Section 3.1.3, which includes 700 segments. For SNR at each level between $6 \mathrm{~dB}$ and $12 \mathrm{~dB}$, we generate 100 segments with random transition time, evaluate all four approaches using these data, and take the average of the results.

Figure 4.10 (a) shows that the onset error of these four methods. Note that the increasing trend of these curves in the figure does not mean a decrease of performance since the absolute error becomes smaller with the increase of SNR. To better understand the impact of SNR, in Figure 4.10 (b), we show the absolute onset error of these methods. Form Figure 4.10 (b), we can see that although Bonato and Hodges' approaches have some oscillation, all methods tend to have smaller absolute mean error when SNR is higher. 


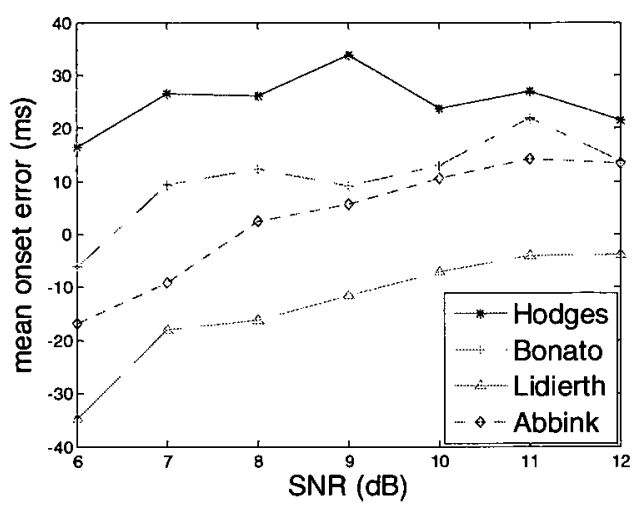

(a)

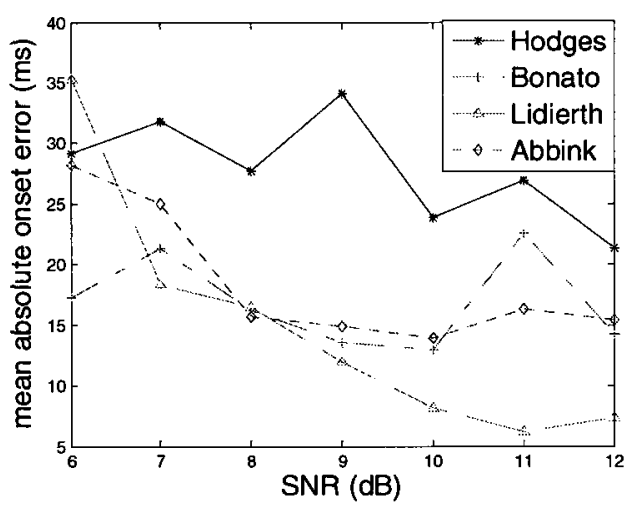

(b)

Figure 4.10 Effect of SNR to the mean of (a) onset error and (b) absolute onset error.

Figure 4.11 (a) shows the standard deviation of onset error and Figure 4.11 (b) shows that of the absolute onset error of these four approaches, which are almost same to each other. Figure 4.11 shows Hodges, Abbink and Lidieth have smaller standard deviation with the increase of SNR, while Bonato has a deviation at $11 \mathrm{~dB}$. This may simply be the result of an outlier.

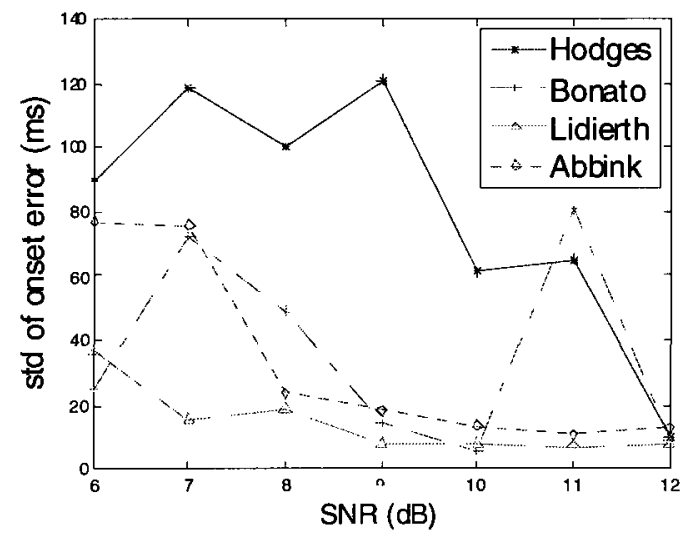

(a)

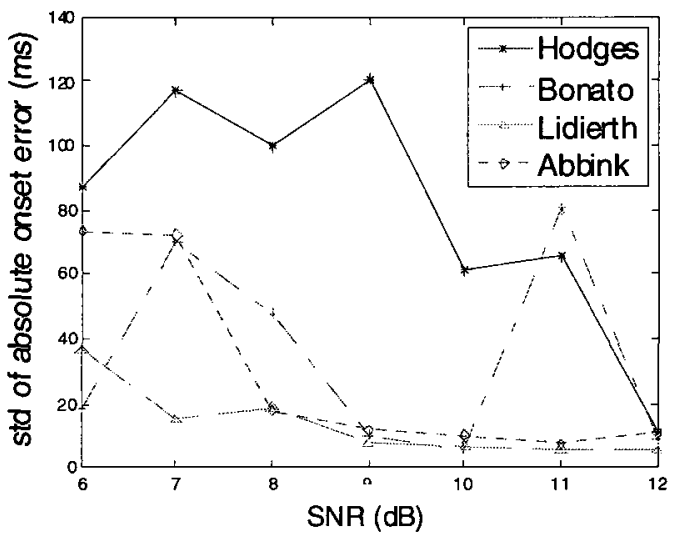

(b)

Figure 4.11 Effect of SNR to the s.t.d. of (a) onset error and (b) absolute onset error. 
The impact of the transition time to onset detection is evaluated using Constant SNR and Random Transition Time generated in Section 3.1.4. For transition time at each level between $5 \mathrm{~ms}$ and $30 \mathrm{~ms}$, we generate 100 segments with random SNR value, evaluate all four approaches using these data, and take the average of the results.

Figure 4.12 (a) and (b) show the mean of onset error and absolute onset error, respectively. All methods seem to show an increase in absolute onset error with increasing transition time, except for Liderth, which exhibits a decrease, albeit a small one. The mean absolute onset error of the Liderth approaches zero with increasing transition time, suggesting that the bias is being reduced with the increased transition time.

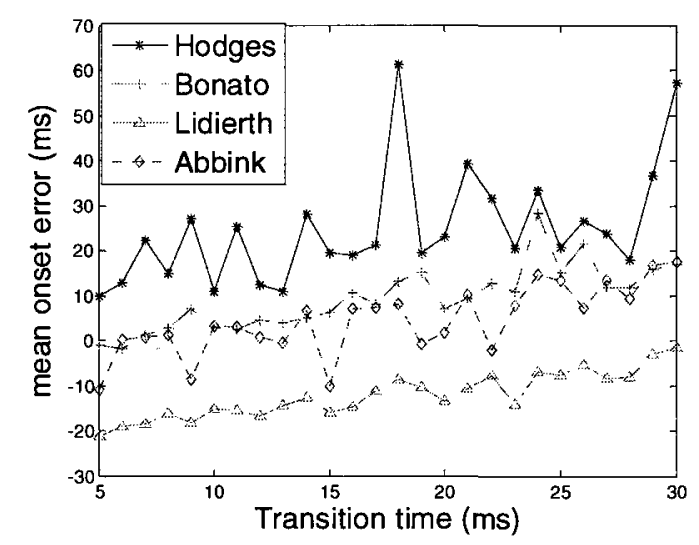

(a)

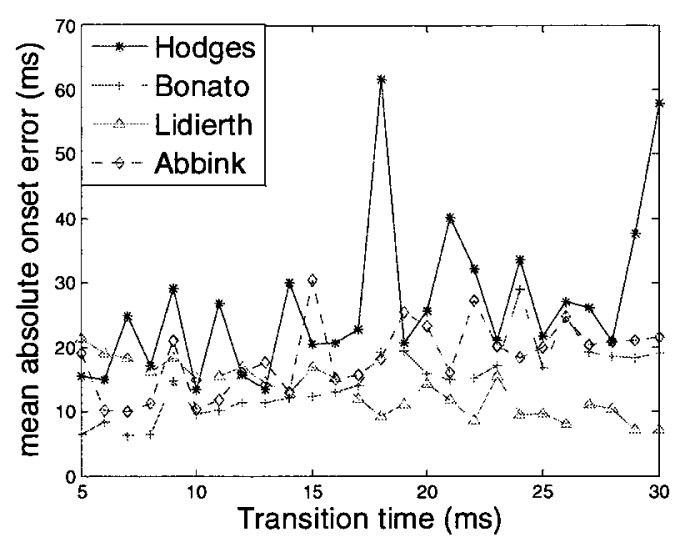

(b)

Figure 4.12 Effect of ramp duration to mean of (a) onset error and (b) absolute onset error.

Figure 4.13 (a) and (b) show the standard deviation of onset error and absolute onset error, respectively. These two figures are almost identical to each other, and the figure shows that the error standard deviation curves of these approaches are either constant 
(e.g., Lidierth) or quite random (e.g., Hodges). Thus, the standard deviation of detection error is not sensitive to the transition time.

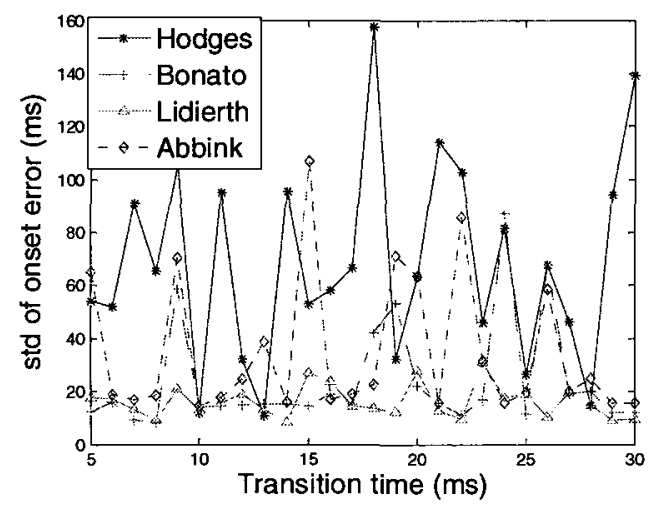

(a)

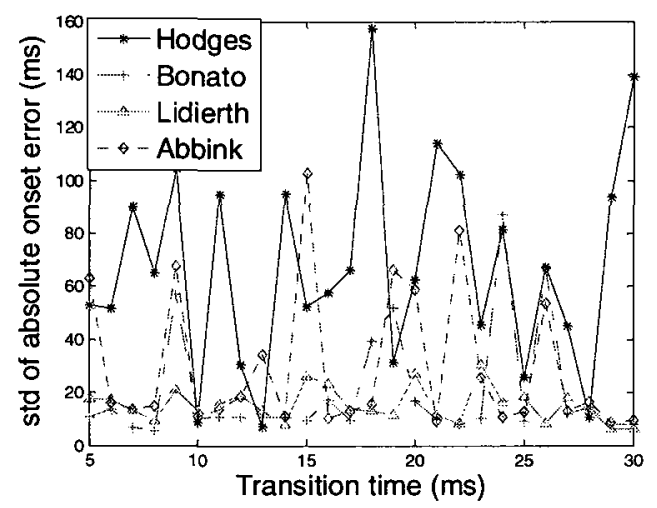

(b)

Figure 4.13 Effect of ramp duration to s.t.d. of (a) onset error and (b) absolute onset error.

\subsubsection{Discussion}

From the above analysis, we can conclude that Bonato and Lidierth outperform the other two approaches, with smaller mean error and smaller standard deviation, and also higher $\mathrm{P}_{100}$. Since Lidierth approach uses the same post-processor as Bonato approach, it is not surprising to see they have similar performance. Abbink's approach has the same conditioning and detection unit as Hodges approach, but it has more complex postprocessor than Hodges approach. Thus, Abbink was expected to perform better than Hodges approach.

On the other hand, Hodges method is very simple in its implementation and its performance may be adequate for certain applications. Although the Hodges method is not the best detector, it should still be considered for applications where computing 
power or time is an important concern, such as in embedded real-time systems, where computational power is limited. For the majority of the applications, the extra complexity of the other methods is negligible.

\subsection{Algorithm Evaluation on Real Data}

\subsubsection{Evaluation on Real Data}

In this section, we use the real data to evaluate the above four approaches. As shown in Figure 4.14 (a), the accuracy function increase quite slow, and Figure 4.14 (b) shows that the error also distributes wider than that using synthetic data.

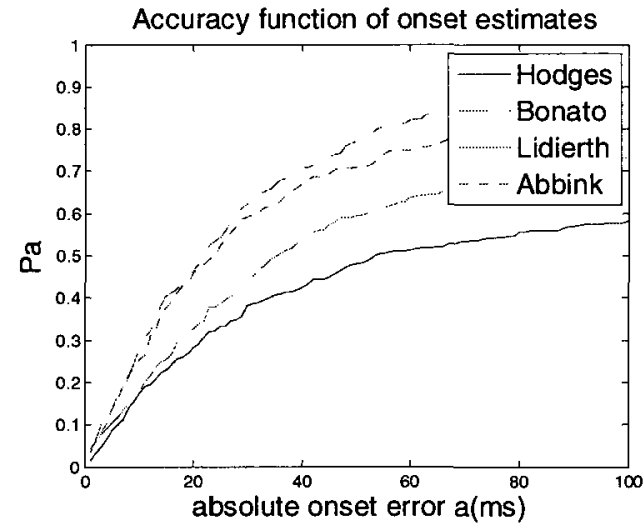

(a)

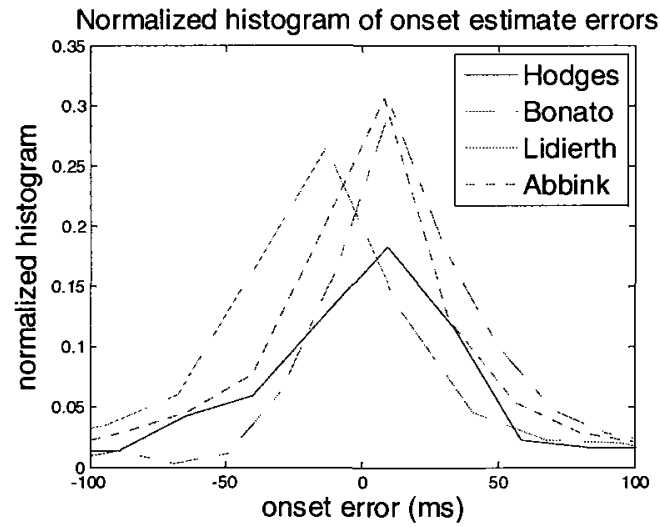

(b)

Figure 4.14 (a) Accuracy function and (b) normalized histograms of all approaches on real data.

We also show the mean, standard deviation and other metrics of these four approaches in Table 4.2. The negative mean error in the table refers to the scenario that 
the estimated onset time is behind the actual time, and the positive value means the estimate is prior to the actual time.

Table 4.2: More comparison results of detection error for four approaches on real data.

\begin{tabular}{ccccc}
\hline Approaches & Hodges & Bonato & Lidierth & Abbink \\
\hline Mean error(ms) & 186 & 30 & 67 & 16 \\
S.T.D. error (ms) & 300 & 86 & 237 & 119 \\
Skewness (ms) & 3.44 & 3.67 & 3.96 & 4.01 \\
Detection rate (\%) & $98.61 \%$ & $100 \%$ & $96.39 \%$ & $97.22 \%$ \\
$P_{100}$ & 0.5803 & 0.9111 & 0.7320 & 0.8600 \\
\hline
\end{tabular}

\subsubsection{Discussion}

The experimental results show that the Bonato method has the best performance and the Abbink method has the second good performance. The Liderth has the third performance and Hodges is the worst.

It is not surprising to see that the double threshold approaches over perform the single threshold approach, although the implementation of these double thresholds approaches might be tricky. Among the double threshold approaches, Bonato has the best performance, which might be due to the effect of whitening filter in its conditioning system. In research conducted in the following chapter, we can see how Bonato's conditioning system helps improve its detection performance.

The standard deviation of the onset determined manually was $34 \mathrm{~ms}$, which is much smaller than the automated methods. As such the automated approaches are inferior to manual onset detection and further improvements are necessary. 


\subsection{Conclusions}

Based on the experimental results from both synthetic data and real SEMG signal, we can see that these approaches have much better performance on synthetic data than on real SEMG signal. One major reason is that the synthetic data has the stationary characteristics while it is often hard to guarantee in real data. Another reason is that the reference onset time in the real data is from human inspector, which might cause extra error.

From the analysis, we conclude that the double threshold approaches have better performance than the single threshold approach, from accuracy function to the statistical value such as mean and standard deviation. However, the flexibility of double thresholds approaches also introduces its vulnerability, since different implementation approach will dramatically influence the performance accuracy. 


\section{Chapter 5 Effect of High Pass Filtering}

The general processing of SEMG signal usually involves four steps [55], which are amplification, band pass filtering, full wave rectification, and subsequent low pass filtering. The low pass filter (LPF) in the second step is for noise attenuation, while the LPF in the fourth step serves for the purpose of replicating the second order response of the muscle to the impulses provided by the MUAPs [55]. Amplification and band pass filtering are often incorporated into the front-end circuit of data collection and preconditioning system. Full wave rectification and subsequent LPF are often included into a conditioning and estimating system and were first implemented by Inman [33] in 1952 as the first continuous EMG amplitude estimator.

The band-pass filter, applied to reduce noise and artifacts, consists of a LPF and a high pass filter (HPF). The LPF is often used to remove unwanted amplitude modulation due to sliding of the innervation zone below the electrodes, while the HPF is used to get rid of movement artifacts that are often with a lower frequency.

In this chapter, we analyze the influence of HPF to the SEMG signal processing using four threshold-based onset detection approaches. Experimental results and analysis are based on real SEMG signal. 


\subsection{Introduction of HP Filtering}

HPF is important to SMEG signal processing based on the following observations. First, during prolonged isometric contractions, the amplitude of SEMG signal could increase without the increase of muscle force. In the frequency domain, this phenomenon results in a modest decrease in high frequency power and a relatively large increase in low frequency power, which leads to the claim that the signal in the low frequency range could have little relationship to force in many cases. Indeed, [43] suggests that a high pass filtered SEMG provides a superior estimate of force. This is also consistent with [66], which found a better correlation of force for spectral components in the high frequencies.

Second, researchers found that temporal whitening filter SEMG signal could improve the accuracy of amplitude estimates [13], [28]. Temporal whitening is often applied to remove the predictable components that are found most in the low frequency range. From this perspective, whitening is similar to high pass filtering at removing signals at frequency lower than $200 \mathrm{~Hz}$ [55]. Since whitening filter is more complex in terms of design and implementation comparing to high pass filter, high pass filtering attracts lots of interest in the field of SEMG signal processing [42], [32], [55] .

Onset of EMG activity is associated with a change in contractile force, which is associated with high frequency components in the EMG. It is hypothesized that applying a HPF to the EMG will provide a signal that will have better onset detection accuracy. 
To have a subjective inspection on the influence of HPF on SEMG signals, we apply a six order Butterworth HPF with cutoff frequency $20 \mathrm{~Hz}$ to the real SEMG signal. Figure 5.1 shows the magnitude and phase response of this Butterworth filter.

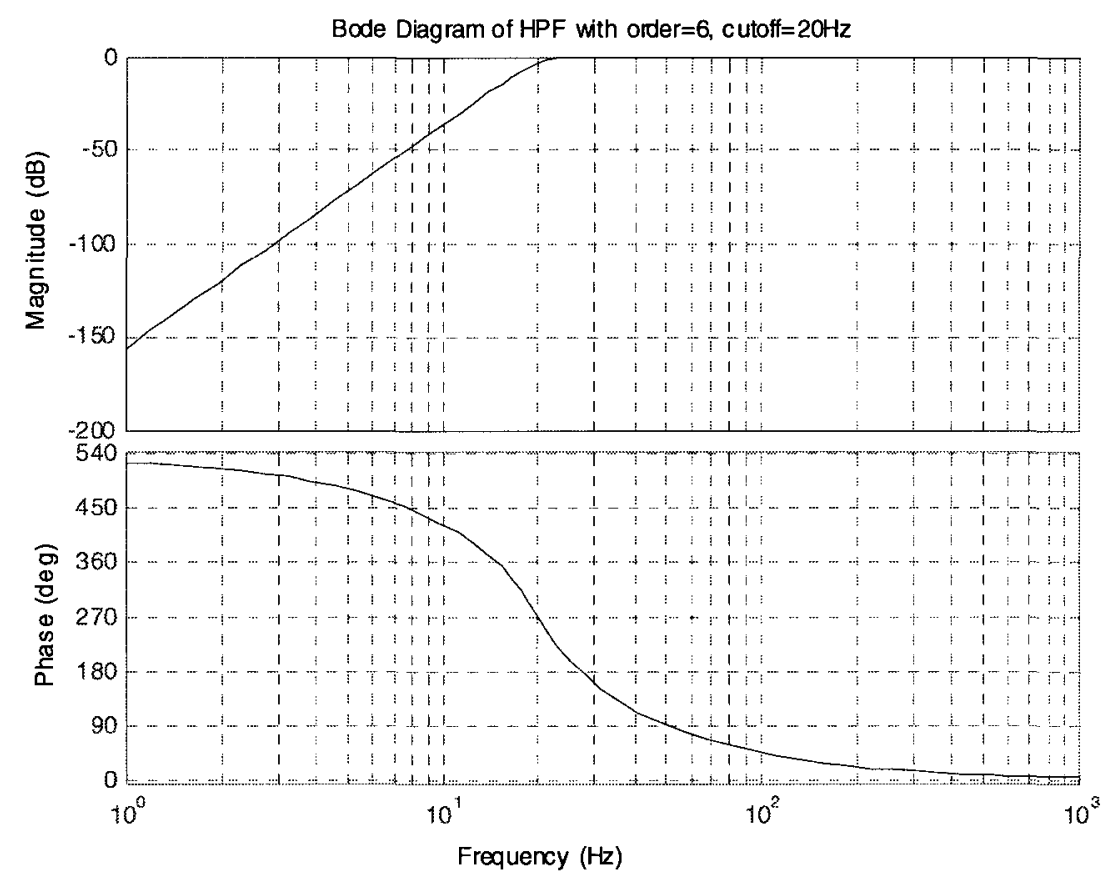

Figure 5.1 Magnitude and phase of the high pass filter.

Figure 5.2 shows the $25^{\text {th }}$ segment of the real data and its results after HPF. From Figure 5.2 (a), we can see there is irrelevant low frequency signal in the original signal, potentially related to motion artifact. As a result, the onset time is hard to determine. In Figure 5.2 (b), the signal amplitude change is more obvious around the onset time in the filtered data. 


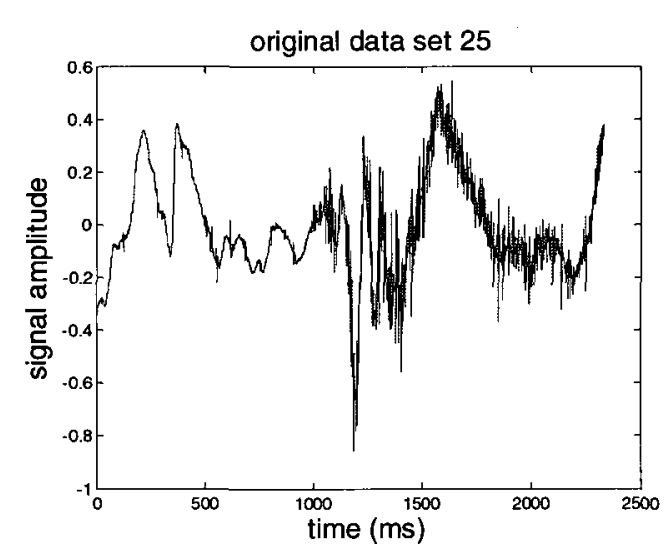

(a)

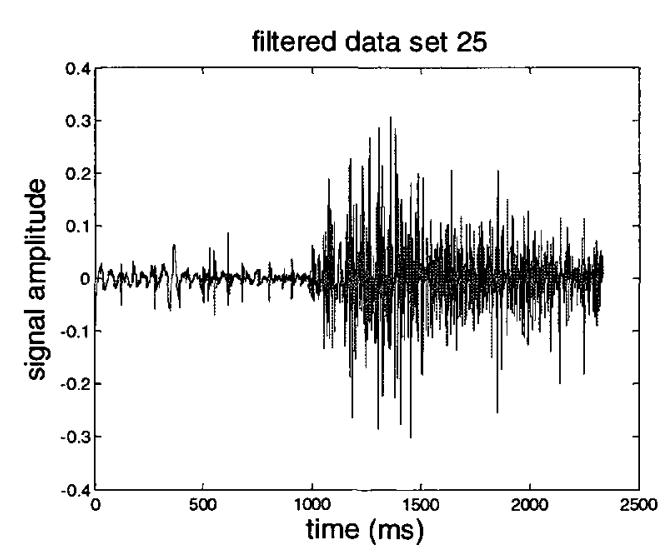

(b)

Figure 5.2 (a) Raw and (b) filtered SEMG signal with a $6^{\text {th }}$ Butterworth HPF with cutoff frequency $20 \mathrm{~Hz}$.

We also compare the original and filtered synthetic data in Figure 5.3, in subfigure (a) and subfigure (b), respectively. These two subfigures are almost same, which means that the influence of HPF is quite small on synthetic data compared to the real data. This is because the relationship between amplitude and force in the real data differs from the synthetic data. In synthetic data, the amplitude of all frequencies changes is controlled in a predictable way, as we increase the variance of the input white noise process around onset time in this work. The force relationship does not change with frequency and there are no low frequency artifacts. Thus, we will analyze the influence of HPF on real data only. 


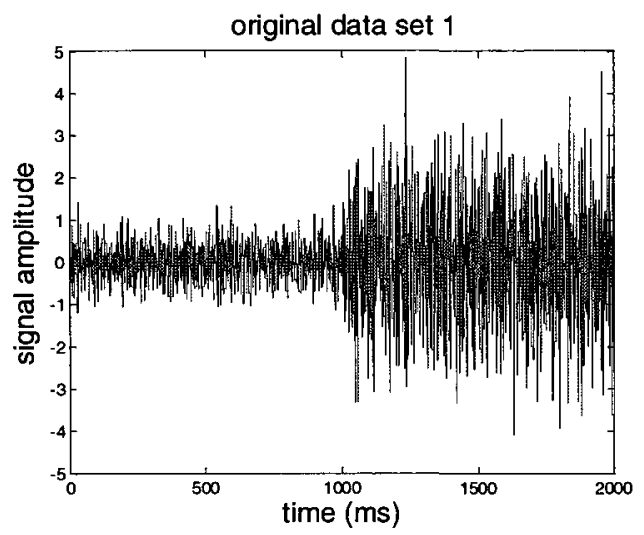

(a)

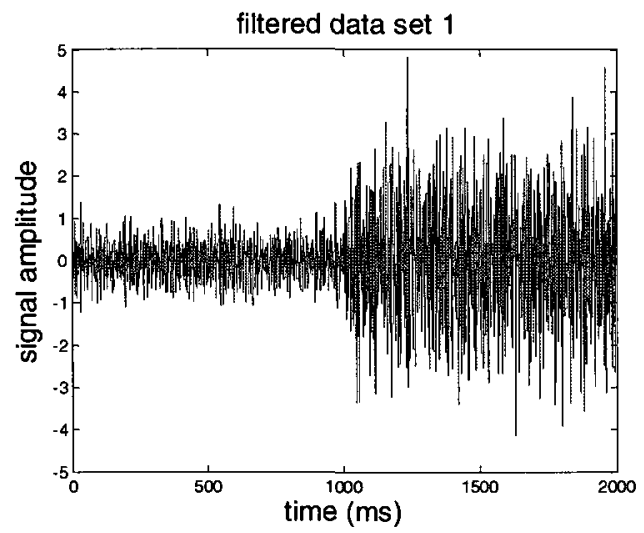

(b)

Figure 5.3 (a) Raw and (b) HP filtered synthetic data with a $6^{\text {th }}$ Butterworth HPF with cutoff frequency $20 \mathrm{~Hz}$.

In the band pass filter, the cutoff frequency of the LPF is relatively stable (e.g., around $450 \mathrm{~Hz}$ [44]), while the cutoff frequency of the HPF could be of different ranges based on different concerns. For example, the SEMG signal often shows slow variations due to unexpected movement and instability of the electrode-skin interface. These unwanted signals are usually in the frequency range 0 to $20 \mathrm{~Hz}$ and the $\mathrm{HPF}$ could be designed with cutoff frequency in a $15-20 \mathrm{~Hz}$ range to remove this noise [44]. Moreover, the cutoff frequency could be at $25-30 \mathrm{~Hz}$, in order to remove the information in the 15 to $20 \mathrm{~Hz}$ range of SEMG spectrum, which includes the firing rates of the active MUs that may be irrelevant in many applications (e.g., in movement analysis). In addition, the spectrum of the point process is not constant in a frequency region below $30 \mathrm{~Hz}$ [44], and high-pass filtering with cutoff frequency higher than $30 \mathrm{~Hz}$ could reduce the influence of the firing patterns of the MUs on the SEMG power spectrum. In [43], an extremely high 
cutoff frequency was used to obtain a better amplitude force-relationship. Therefore, how to choose the cutoff frequency is an important topic.

\subsection{Analysis Method}

\subsubsection{Filter Order}

Before we investigate the influence of cutoff frequency, we need to find an appropriate analysis method since the order of the filter and other conditioning procedures all affect the results.

In Figure 5.4, we show the magnitude and phase of HPF with different order and cutoff frequency. We choose the filter order 1 and 6 , due to their popularity [55]. Figure 5.4 (a) and Figure 5.4 (b) have same cutoff frequency but different order. The figure shows that with higher order, its magnitude response is steeper and has less transition period around its cutoff frequency, which usually results in better performance. However, the difference between their magnitude responses is not dramatic. On the other hand, Figure 5.4 (a) and Figure 5.4 (c) have the same filter order but different cutoff frequency, and their magnitude responses are obviously different. From Figure 5.4, we can see that the cutoff frequency has more influence on signal than the order has. Thus, we focus on analyzing HPFs with different cutoff frequency and use a filter order of 6 throughout this work. 


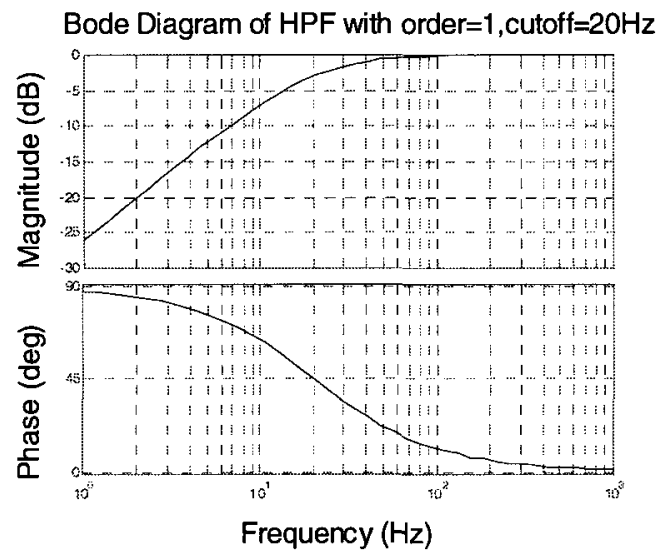

(a)

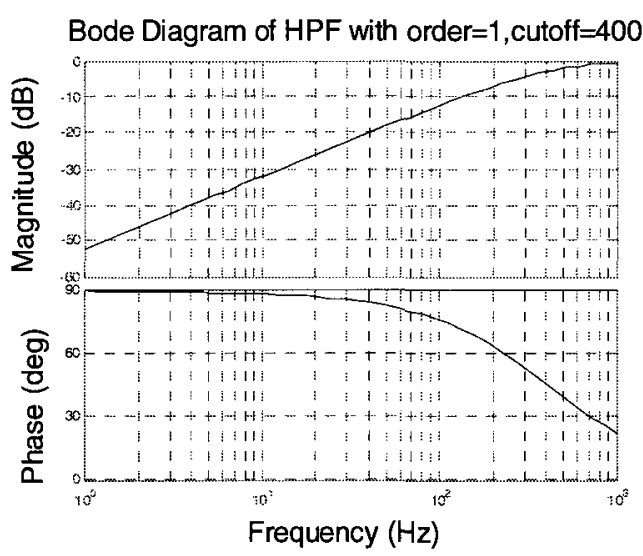

(c)

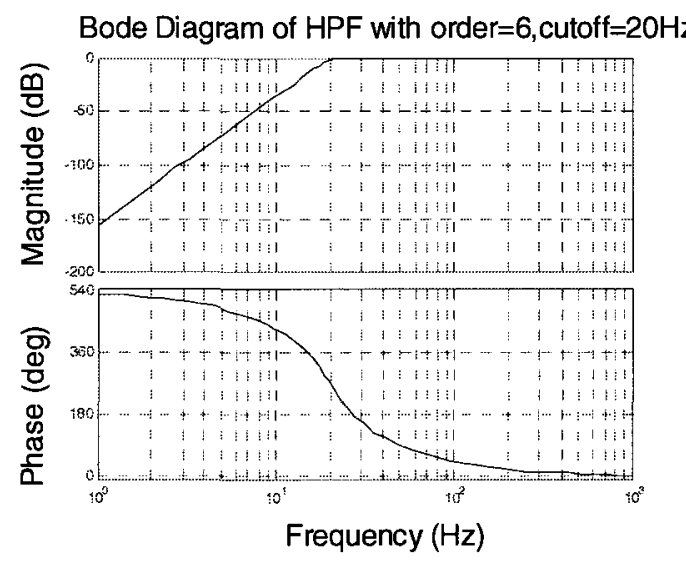

(b)

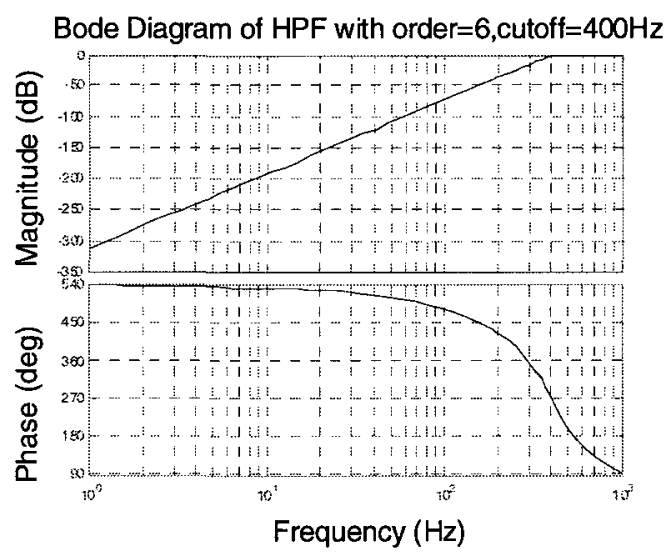

(d)

Figure 5.4 Magnitude and phase for high pass butter worth filter, with (a) order=1, cutoff $=20 \mathrm{~Hz}$; (b) order $=6$, cutoff $=20 \mathrm{~Hz} ;$ (c) order $=1$, cutoff $=400 \mathrm{~Hz} ;(\mathrm{d})$ order $=6$, cutoff $=\mathbf{4 0 0 H z}$.

\subsubsection{Filter Cutoff Frequency}

In [55], the authors tried various HPFs, using the root mean square (RMS) difference and the correlation between the HP filtered EMG signal and a normalized LP filtered data as criteria to pick the optimal design for high pass filter. Its implementation procedures 
include a LPF with $500 \mathrm{~Hz}$ cutoff frequency, a HPF with cutoff frequency range of $20 \mathrm{~Hz}$ to $440 \mathrm{~Hz}$, rectification and subsequent LPF, linear and nonlinear normalization.

However, not all the procedures are applicable or necessary in practice. For example, linear and nonlinear normalization are not necessary in this work since all approaches under analysis are using absolute thresholds instead of relative ones. The first LPF with cutoff frequency $500 \mathrm{~Hz}$ could be skipped since it is actually an all-pass filter to our real data, given half of the sampling frequency is $500 \mathrm{~Hz}$. Moreover, subsequent lowpass filtering may not benefit the performance of these approaches as well since each approach has its own conditioning system.

To justify the above claim on certain procedures in the system, we conduct two experiments, one is to use HPF only and another is using HPF plus an extra LPF. In the first experiment, we process the raw SEMG data using the $6^{\text {th }}$ order Butterworth HPF with cutoff frequency $20 \mathrm{~Hz}$ and no other preconditioning process.

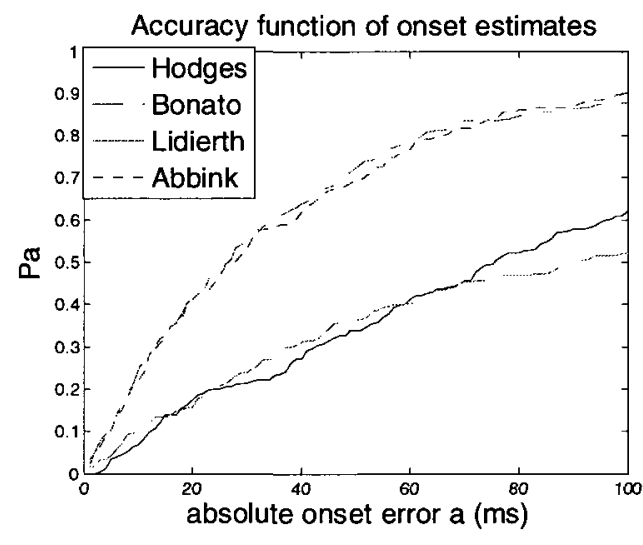

(a)

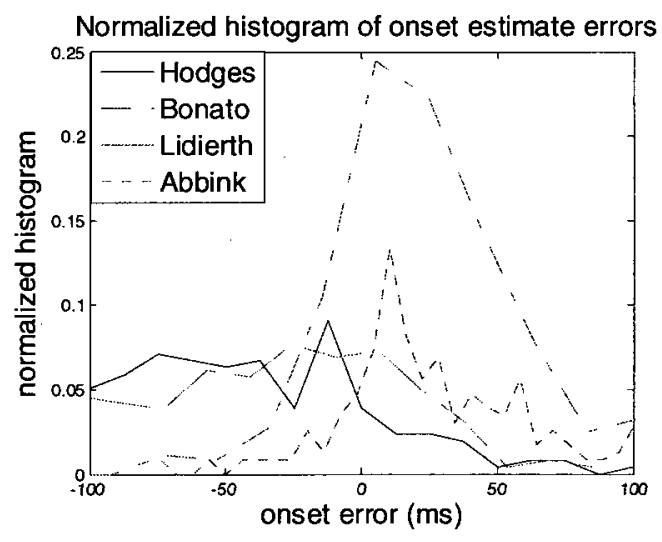

(b)

Figure 5.5 (a) Accuracy function and (b) normalized histograms of all approaches using HPF in conditioning system. 
In Figure 5.5 (a), the accuracy function has lower $\mathrm{P}_{100}$ value for all approaches comparing to Figure 4.7 (a). Also the normalized histogram in Figure 5.5 (b) is lower and less centered than that in Figure 4.7 (b).

In Table 5.1, we show more evaluation results of the estimate error. Comparing it to Table 4.2, we can see that the mean error of all approaches except Hodges is worse than those in Table 4.2. In addition, the detection rate of all approaches drops a lot for all approaches except Bonato. This is because the other three approaches use LPF instead of HPF in their conditioning system while Bonato uses whitening filter that also removes unwanted low frequency data [55].

Table 5.1: More comparison results of detection error for four approaches using HPF.

\begin{tabular}{ccccc}
\hline Approaches & Hodges & Bonato & Lidierth & Abbink \\
\hline Mean error(ms) & -104 & 59 & -132 & 35 \\
S.T.D. error (ms) & 112 & 132 & 150 & 47 \\
Skewness (ms) & 1.35 & 3.32 & 1.14 & 2.15 \\
Detection rate (\%) & $70.56 \%$ & $100 \%$ & $72.22 \%$ & $64.17 \%$ \\
$\mathrm{P}_{100}$ & 0.6220 & 0.8778 & 0.5192 & 0.9004 \\
\hline
\end{tabular}

To evaluate the impact of HPF to the approaches using LPF in their conditioning system, we apply an extra a $1^{\text {st }}$ order LPF with cutoff frequency $1 \mathrm{~Hz}$ after the HPF. The results are shown in the following figure and table. 


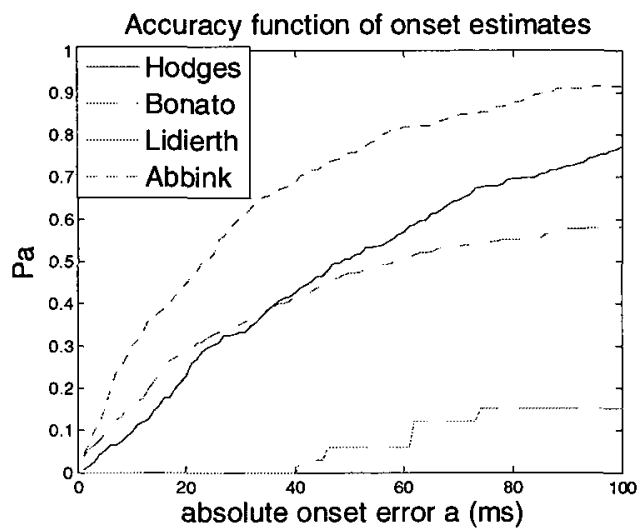

(a)

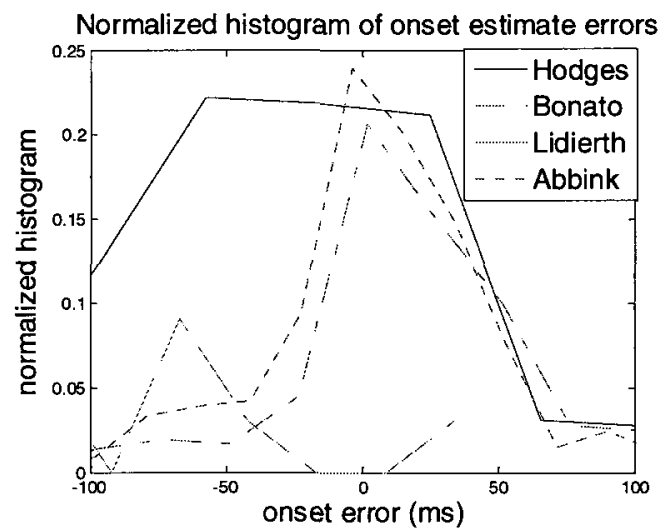

(b)

Figure 5.6 (a) Accuracy function and (b) normalized histograms of all approaches using HPF and extra LPF in conditioning system.

Table 5.2: More comparison results of detection error for four approaches using HPF+LPF.

\begin{tabular}{ccccc}
\hline Approaches & Hodges & Bonato & Lidierth & Abbink \\
\hline Mean error(ms) & -44 & 318 & -438 & 9 \\
S.T.D. error (ms) & 125 & 419 & 344 & 84 \\
Skewness (ms) & 3.11 & 3.25 & 0.92 & 3.21 \\
Detection rate (\%) & $81.39 \%$ & $99.72 \%$ & $9.17 \%$ & $91.67 \%$ \\
$\mathrm{P}_{100}$ & 0.7713 & 0.5822 & 0.1515 & 0.9152 \\
\hline
\end{tabular}

Figure 5.6 and Table 5.2 show that the performance of Bonato and Lidierth is degraded than the results using HPF only. This is because Bonato approach does not need LPF in conditioning system and Lidierth approach uses a LPF that has much higher cutoff frequency (50 times higher) than the one used in this test. However, the performance of Hodges and Abbink is better than that using HPF only, since their LPF has similar cutoff frequency $(3 \mathrm{~Hz})$ to the one applied here. 
From the above experiments, we can tell that using HPF with similar conditioning system to the original ones in each approach have better performance than using HPF only and we will show the experimental results later.

\subsection{Results \& Analysis}

In this section, we show the performance of these four approaches using HPF at different cutoff frequency as pre-conditioning system, the cutoff frequency ranges from $10 \mathrm{~Hz}$ to $450 \mathrm{~Hz}$, with increase step $10 \mathrm{~Hz}$.

Figure 5.7 shows that all approaches have very high detection rate except Bonato, which has detection rate decreased when cutoff frequency is not between 20 and $370 \mathrm{~Hz}$. This is because Bonato uses whitening filter in its conditioning system, high cutoff frequency will filter out the useful information for Bonato method.

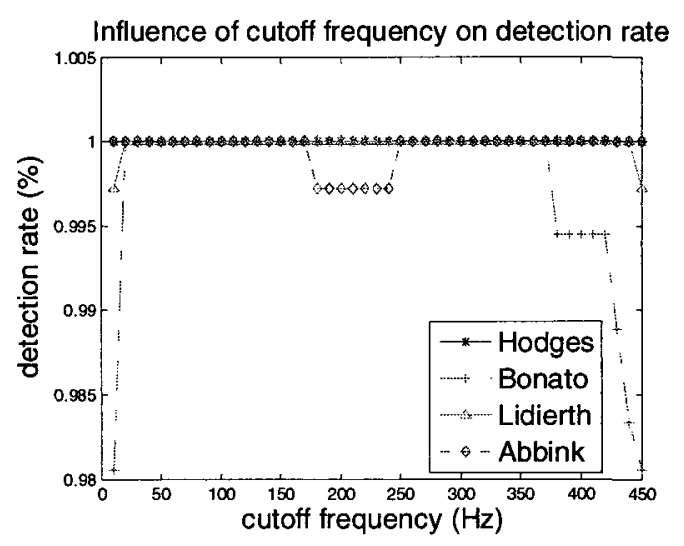

Figure 5.7 The influence of cutoff frequency on the detection rate.

We also evaluate the influence of cutoff frequency on the mean and standard deviation of onset detection error. As shown in Figure 5.8 (a), Lidierth has the lowest mean error comparing to other approaches and it achieves its minimum value at cutoff 
frequency $410 \mathrm{~Hz}$. Figure 5.8 (b) and Abbink has the lowest standard deviation of the detection error, with minimum value at cutoff frequency $240 \mathrm{~Hz}$.

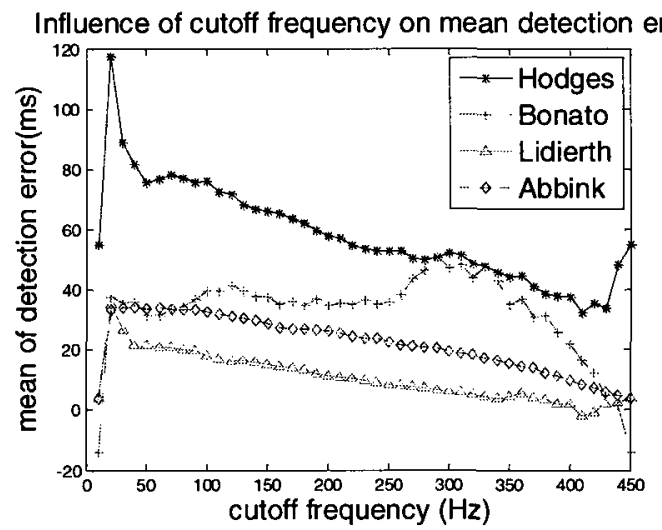

(a)

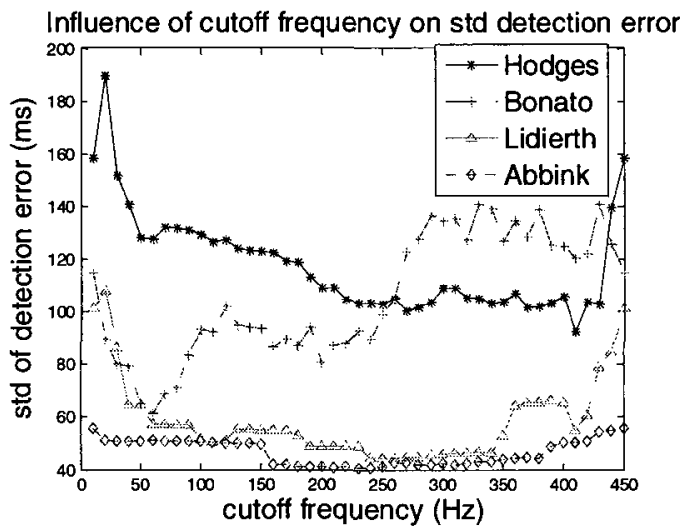

(b)

Figure 5.8 The influence of cutoff frequency on the (a) mean and (b) standard deviation of the onset detection error.

Figure 5.9 (a) and (b) show the influence of cutoff frequency on the skewness and $\mathrm{P}_{100}$.

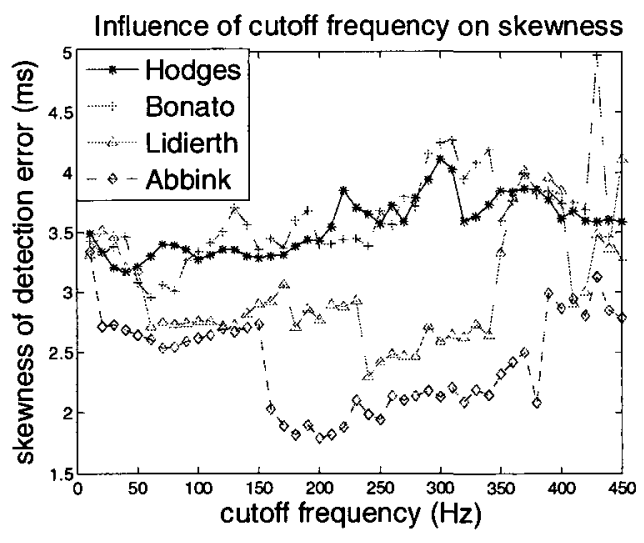

(a)

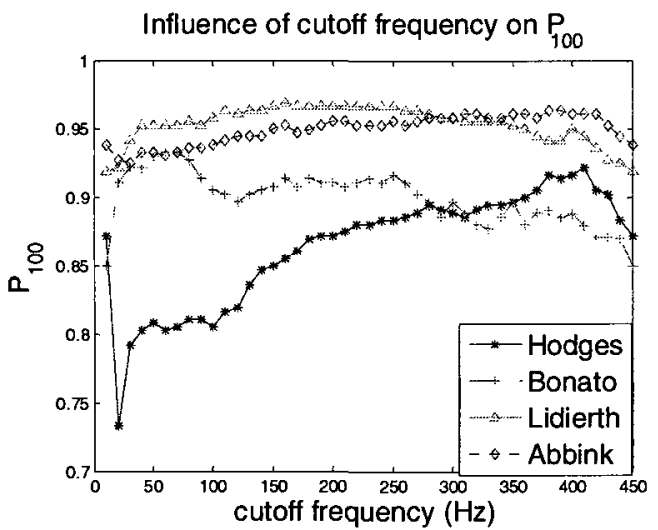

(b)

Figure 5.9 The influence of cutoff frequency on the (a) skewness and (b) $P_{100}$ of the onset detection error.

Figure 5.9 (a) shows that Abbink has the most symmetric detection error histogram, with the lowest skewness value around cutoff frequency $200 \mathrm{~Hz}$ and achieves the highest 
value of $\mathrm{P}_{100}$ at cutoff frequency $30 \mathrm{~Hz}$. Figure 5.9 (b) shows that Lidierth has average higher value at $\mathbf{P}_{100}$ than other approaches.

From these results, we can see that there is no single universal optimal HPF cutoff frequency that benefits all detection approaches. Instead, each approach should consider its specific conditioning system and detection rule to design this pre-conditioning HPF. We want the HPF helps reduce the mean, standard deviation of the detection error, and increase the detection rate and $\mathrm{P}_{100}$. Skewness with smaller absolute value is preferred but not a major concern in this case.

Ideally, we should have an optimization function to calculate the optimal frequency to achieve optimal value of each metric. In other words, the optimal cutoff frequency should satisfy $\left[f_{1}, y_{1}\right]=\min ($ mean_err $),\left[f_{2}, y_{2}\right]=\min \left(s t d \_e r r\right),\left[f_{3}, y_{3}\right]=\max \left(P_{100 \_e r r}\right)$, and $\left[f_{4}\right.$, $\left.y_{4}\right]=\max \left(\right.$ detect_rate_err), where $y_{1-4}$ is the $\min$ or max value of each function and $f$ is the corresponding frequency. As shown in Table 5.3, we compare $f_{1} \sim f_{4}$ and find the most matching frequency as the optimal cutoff frequency.

Table 5.3: Best cutoff frequency for each judging function.

\begin{tabular}{ccccc}
\hline Approaches & Hodges & Bonato & Lidierth & Abbink \\
\hline$f_{1}(\mathrm{~Hz})$ & 410 & 10 & 410 & 10 \\
$f_{2}(\mathrm{~Hz})$ & 410 & 60 & 250 & 240 \\
$f_{3}(\mathrm{~Hz})$ & 410 & 60 & 160 & 380 \\
$f_{4}(\mathrm{~Hz})$ & {$[10,450]$} & {$[10,370]$} & {$[20,450]$} & {$[10,170],[250,450]$} \\
final decision & 410 & 60 & TBD & TBD \\
\hline
\end{tabular}


We notice that the Lidierth and Abbink have different optimal frequency for different requirement, so we do a second level comparison among $f_{1}, f_{2}$, and $f_{3}$. We do not calculate the corresponding $y$ value of $f_{4}$ here since it is a range. We compare the $y(f)$ at different frequency with the optimal y value to pick up the one with least cost. As shown in Table 5.4, for Lidierth approach, the best cutoff frequency should be $f_{2}$ (i.e., $250 \mathrm{~Hz}$ ), since it can achieve the best performance overall. We use the same methodology to get the best cutoff frequency for Abbink approach, which is $380 \mathrm{~Hz}$.

Table 5.4: Second level composition for best cutoff frequency for Lidierth approach.

\begin{tabular}{|c|c|c|c|}
\hline Frequency $(\mathrm{Hz})$ & $\mathrm{f}_{1}$ & $\mathrm{f}_{2}$ & $f_{3}$ \\
\hline mean_err $(f)-$ mean_err $\left(f_{1}\right)$ & 0 & 10.3167 & 16.4417 \\
\hline$s t d \_e r r(f)-s t d \_e r r\left(f_{2}\right)$ & 10.9050 & 0 & 10.6391 \\
\hline$P_{100 \_e r r}(f)-P_{100 \_e r r}\left(\mathrm{f}_{3}\right)$ & -0.0250 & -0.0028 & 0 \\
\hline
\end{tabular}

Thus, we set HPF cutoff frequency as $410 \mathrm{~Hz}$ for Hodges approach, $60 \mathrm{~Hz}$ for Bonato approach, $250 \mathrm{~Hz}$ for Lidierth approach, and $380 \mathrm{~Hz}$ for Abbink approach. The HPF cutoff frequency of Bonato approach is relatively low comparing to other approaches. This is because Bonato approach uses whitening filter in its original conditioning system. The HPF we added here in combination with the whitening filter appears to be less beneficial to the onset detection for high cutoff frequencies. The whitening filter effectively removes contributions of low frequency signals, which may explain this behaviour. We apply the HPF with these specific cutoff frequencies to each detection approach and have the results shown in Figure 5.10. 


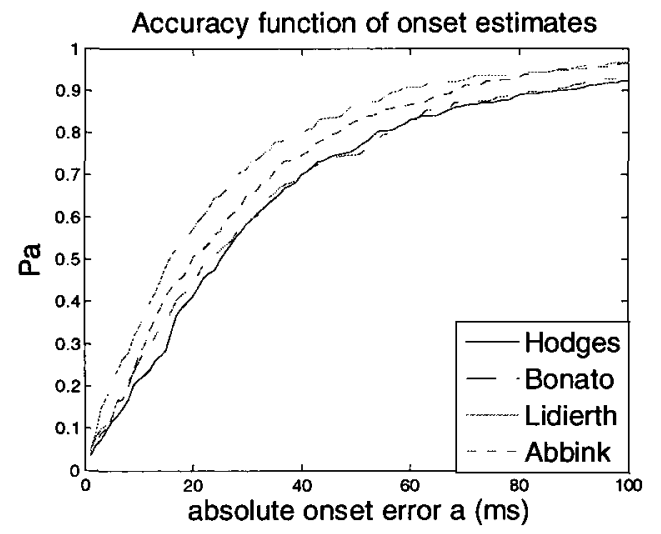

(a)

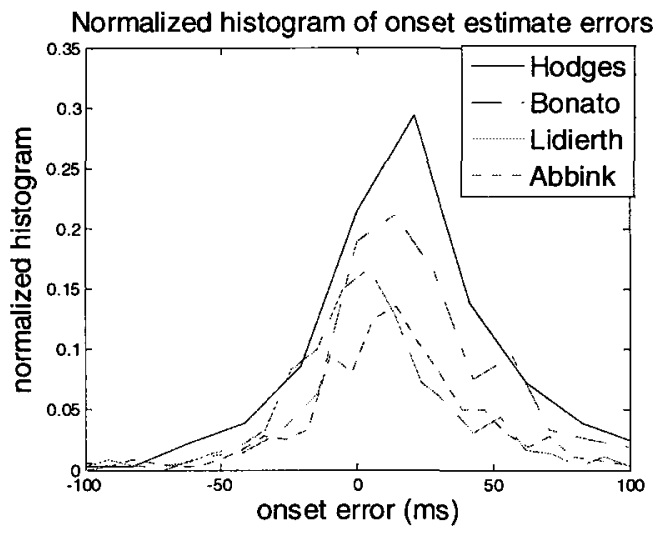

(b)

Figure 5.10 (a) Accuracy function and (b) normalized histograms of all approaches with HPF pre-conditioning.

We also evaluate the performance of each approach using other metrics and show the results in Table 5.5.

Table 5.5: More metrics on detection error for four approaches by using optimal HPF.

\begin{tabular}{ccccc}
\hline Approaches & Hodges & Bonato & Lidierth & Abbink \\
\hline \hline Mean error(ms) & 32 & 31 & 8 & 12 \\
S.T.D. error (ms) & 92 & 61 & 44 & 44 \\
Skewness (ms) & 3.68 & 2.96 & 2.42 & 2.08 \\
Detection rate (\%) & $100 \%$ & $100 \%$ & $100 \%$ & $100 \%$ \\
$\mathrm{P}_{100}$ & 0.9222 & 0.9333 & 0.9667 & 0.9639 \\
\hline
\end{tabular}

The above results are much better than those in Table 5.1 and Table 5.2. Comparing to results in Table 4.2, the performance of these approaches with HPF applied has lower mean error, smaller error standard deviation, higher detection rate and higher value of 
$\mathbf{P}_{100}$. Compared to manual detection, which had a standard deviation of $34 \mathrm{~ms}$, the automated methods are still worse.

\subsection{Conclusion}

From the above analysis, we can conclude that HPF with appropriate cutoff frequency will increase onset detection accuracy. However, optimal selection of the cutoff frequency should be adapted to specific detection approach. As such, the combination of the HPF with existing conditioning system might be tricky in practical implementation. Using a fixed HPF cutoff frequency is possible, and improvements in onset detection can still be realized. 


\section{Chapter 6 Optimal Threshold Selection}

During previous study, we found that a good onset detector/estimator should consider the influence of each factors in the system to achieve optimal performance, such as cutoff frequency in HPF or the thresholds in detection rules. Note that all the approaches we reviewed are threshold based and thus, threshold should be the most influential factor in the whole system. In this chapter, we will analyze the existing detection rules and propose a mechanism to choose the optimal threshold for each detection method.

\subsection{Influence of Threshold to Detection Accuracy}

Both threshold-based and statistical based onset detectors have detection rules and make decision based on the threshold [64]. Thus, threshold is a key factor in onset detection approaches. In this work, we focus at the threshold-based approaches.

Figure 6.1 is a typical example to show the effect of threshold on onset detection accuracy in Lidierth's approach, given the actual onset time $1000 \mathrm{~ms}$. The $\mathrm{x}$-axis is the threshold starting from value 0.5 to value 5. In Figure 6.1 (a), the y-axis is the detected onset time using each threshold on the x-axis; while in Figure 6.1 (b), the y-axis is the output of normalized detection error passing a FIR filter with coefficients $\left[\begin{array}{llll}-1 & 8 & -8 & 1\end{array}\right]$. From the figures, we can see that with different threshold, the detected onset time value varies dramatically, and the estimation error is not a simple mathematically tractable function of threshold. 


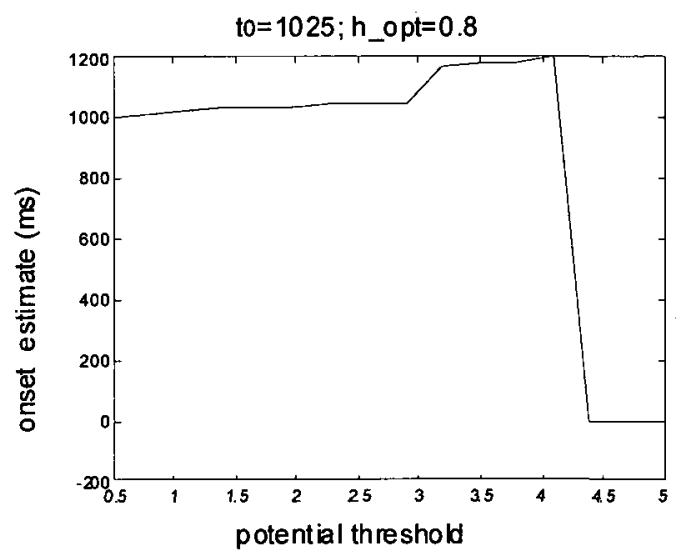

(a)

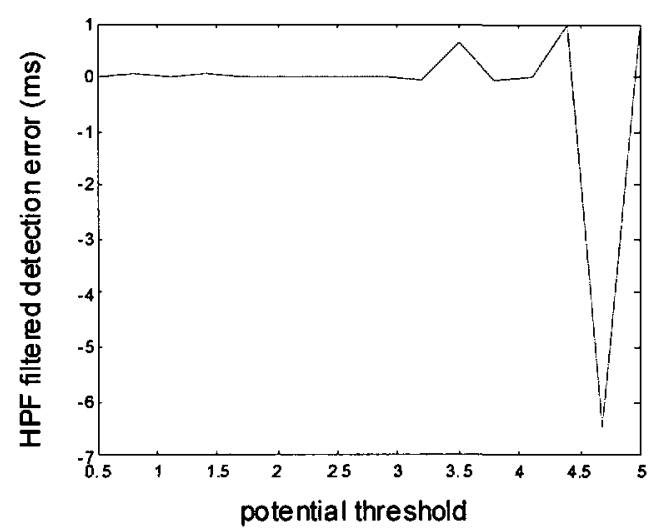

(b)

Figure 6.1 An example of the influence of threshold on (a) onset detection accuracy and (b) HPF filtered detection error.

Therefore, a good threshold is very important to the detection accuracy. In the literature, threshold-based onset detectors/estimators often use a preset threshold to determine the alarm time or the onset time. This kind of design has a major drawback, i.e., the threshold is unable to adapt to signal characteristics or different muscles/forces or different recording systems. For example, the SEMG signal varies from amplitude to frequency, due to the variety of muscle fibers or recording systems. If the threshold is set too low, the detected onset time will be earlier than the actual time. If it is set too high, the detected onset time is behind the actual one or even unable to detect the onset time. Therefore, we need to develop a scheme to choose the optimal threshold and this scheme should satisfy the following requirements: 
- It should be irrelevant to the detection functions. In other words, its procedure should be consistent to different onset estimators. Each onset detection function is only an argument of the threshold-decision algorithm.

- It should be simple and reliable. Since this threshold-decision algorithm is considered as an extra algorithm on top of the onset estimators, its logic should be easy to implement and maintain.

In the following sections, we will discuss how to develop such a scheme. For consistence, we use $g_{k}$ as the decision rule as the four threshold-based approaches use.

\subsection{Detection Function Analysis}

If we use a sign function to describe the relation between threshold and signal, the ideal case would be a stair case. To select a good threshold that just suppresses the noise and detects the onset precisely, we need to have a detection function that shows the obvious level difference between signal and noise. However, in practice, the detection function might vary among detection approaches. Here, we show some examples using the synthetic data. 


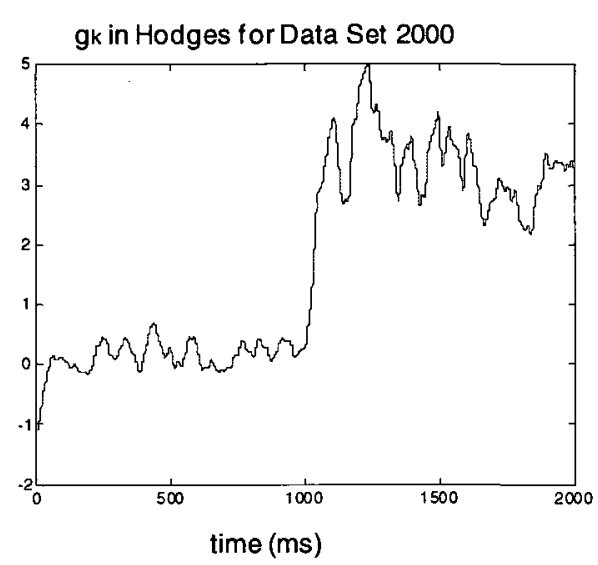

(a)

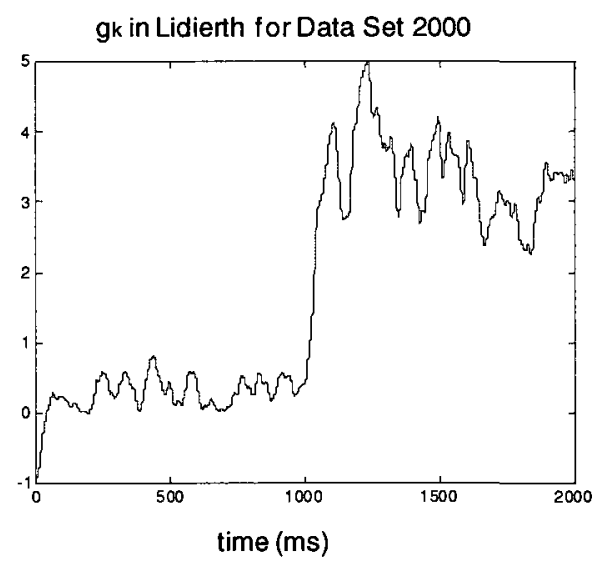

(c)

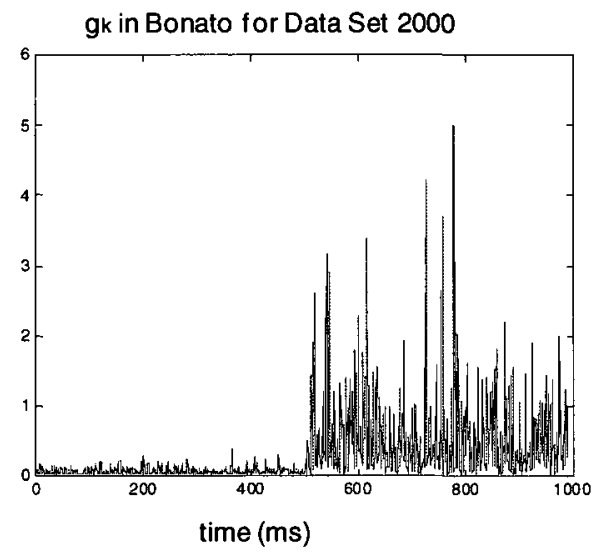

(b)

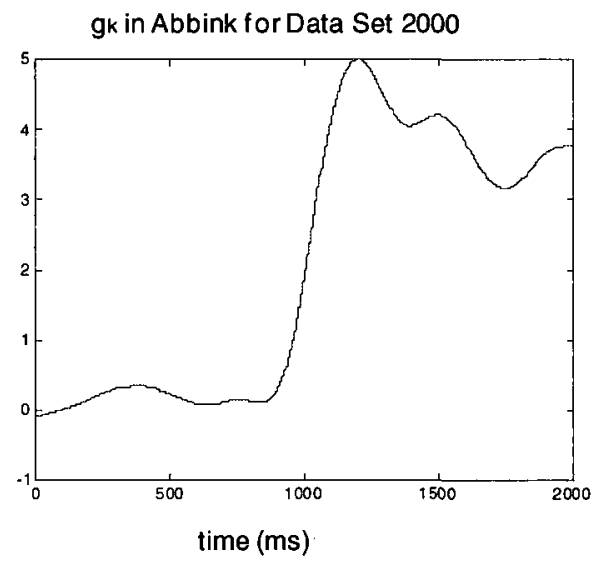

(d)

Figure 6.2 A typical example of detection function for (a) Hodges' approach, (b) Bonato approach, (c) Lidierth approach, (d) Abbink approach.

From Figure 6.2, we can see that most detection function except Bonato approach show the signal after onset has obviously large amplitude comparing to noise and there is lots of noise in Figure 6.2 (b). This means we only need to apply low pass filter to the detection function of Bonato approach, since other detection functions do not need to be extra LP filtered as shown in Section 6.3. 
In Figure 6.3, we evaluate the impact of different low pass filters on the detection function of Bonato approach shown in Figure 6.2 (b).

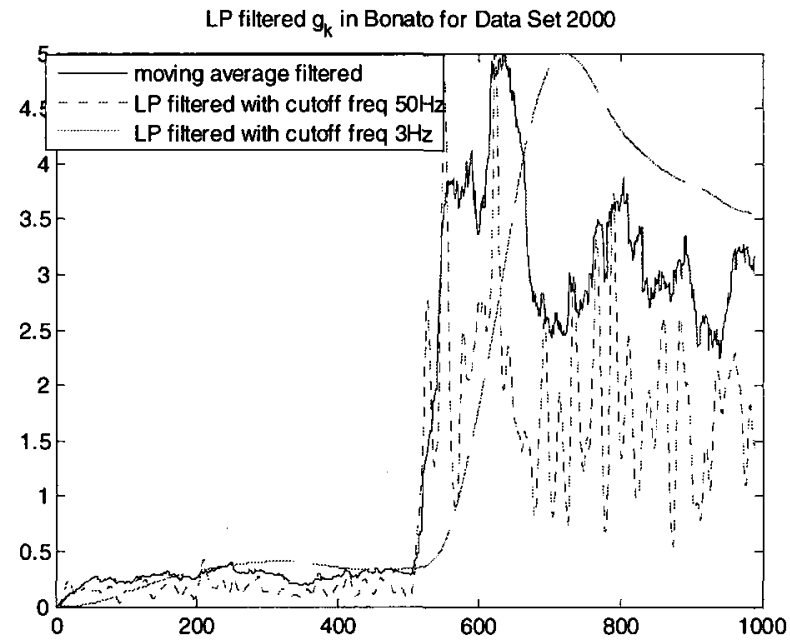

Figure 6.3 The filtered result of the detection function using different low pass filters.

In Figure 6.3, we compare the filtered detection function using three different LP filters: a moving average (MA) filter with window size 50, a Butterworth filter with cutoff frequency $50 \mathrm{~Hz}$, and a Butterworth filter with cutoff frequency $3 \mathrm{~Hz}$. From the figure, we can see that the filtered output of MA filter is good enough to differentiate the noise and the signal, and the result of the $3 \mathrm{~Hz}$ cutoff frequency might over smooth the detection function. Thus, we apply MA filter to the detection function of Bonato approach.

To have a better understanding of these filters, we also show their magnitude and phase response in Figure 6.4. We use "freqz" to draw the magnitude and phase response of MA filter, since it is digital filter. The other two Butterworth filter are analog filter and we directly draw their bode diagram. The sampling frequency is $1000 \mathrm{~Hz}$. 

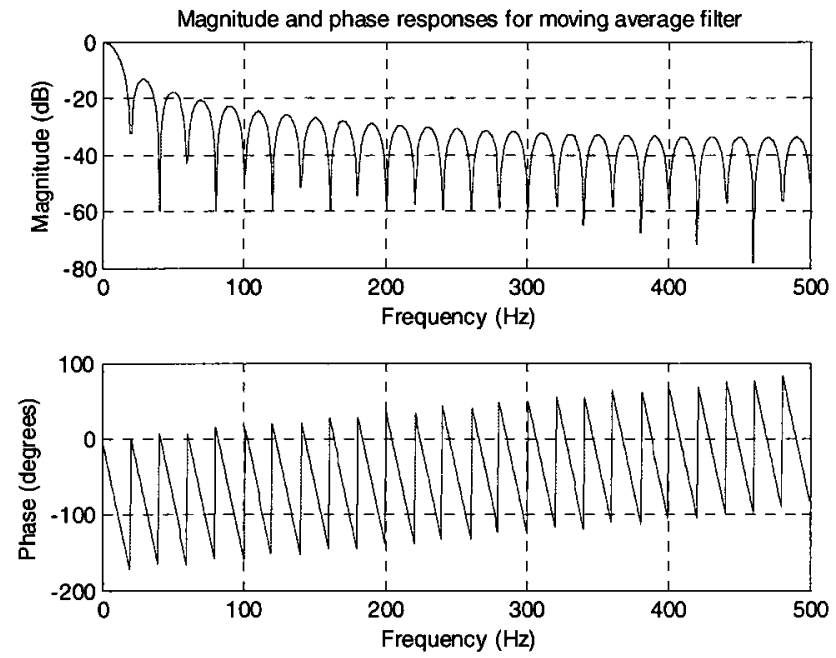

(a)

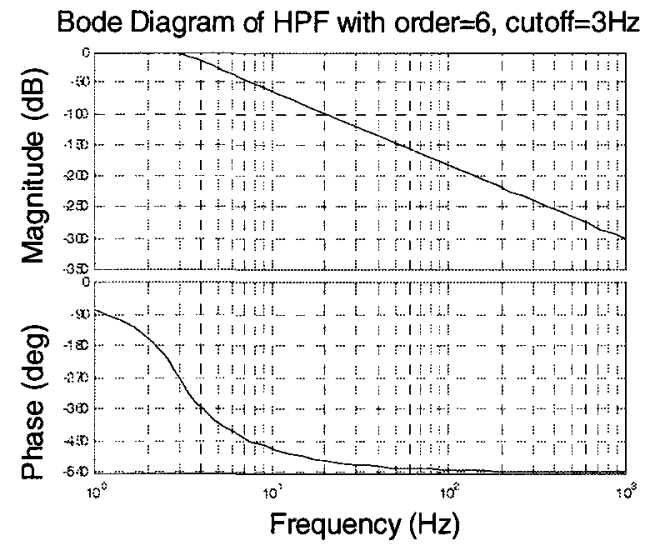

(b)

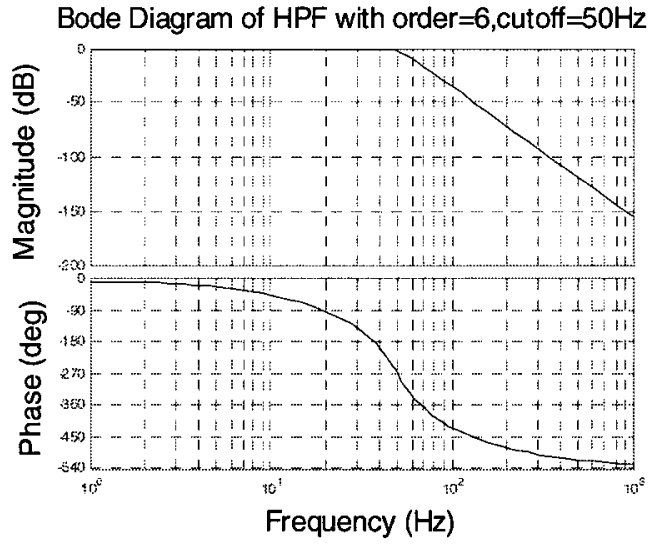

(c)

Figure 6.4 Magnitude and phase response for (a) MA filter, (b) $6^{\text {th }}$ order Butterworth LP with cutoff $3 \mathrm{~Hz}$, (c) $6^{\text {th }}$ order Butterworth LP with cutoff $50 \mathrm{~Hz}$.

Figure 6.4 shows that the magnitude and phase response of Butterworth filter is much smoother than those of MA filter, and the response with cutoff $3 \mathrm{~Hz}$ is much sharper than that with cutoff $50 \mathrm{~Hz}$. However, as shown in Figure 6.3, a MA filter is good enough for the process of detection function for Bonato approach. 


\subsection{Sign Function Analysis}

The idea of selecting a good threshold is conducted by comparing the threshold value with detection functions, in other words, we need to look at the sign function of subtracting threshold from detection function.

Figure 6.5 shows an example of the detection function $g_{k}$ and its smoothed curve from MA filter. We can see that the chosen threshold is above the noise level but below the signal level in Figure 6.5 (a) and its sign function is a two-state function as shown in Figure $6.5(\mathrm{~b})$.

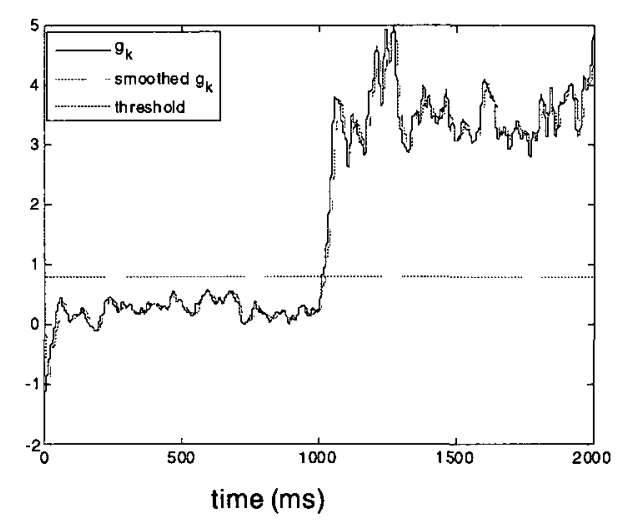

(a)

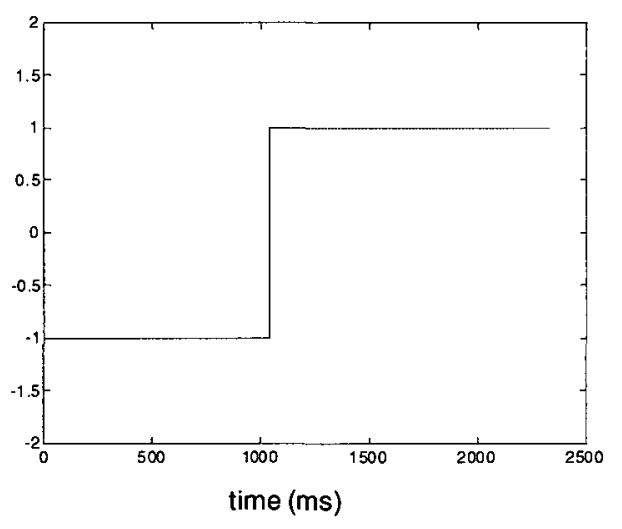

(b)

Figure 6.5 An example of (a) detection function and (b) its sign function of threshold $h_{0}$.

Other cases that can not simply use an on-off two state machine to decide the optimal threshold are shown from Figure 6.6 to Figure 6.8. 


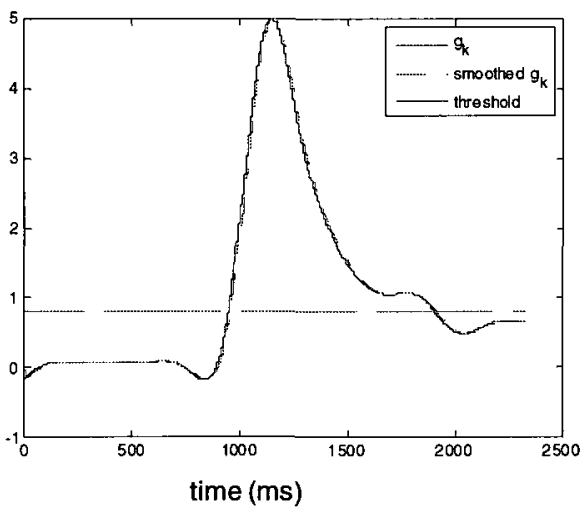

(a)

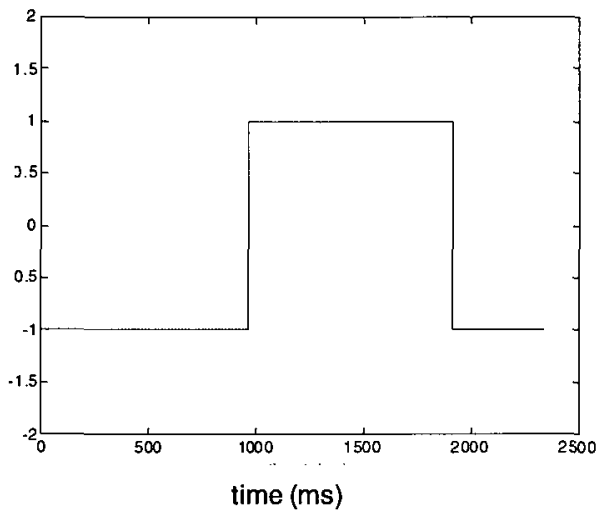

(b)

Figure 6.6 Another example of (a) detection function $g_{k}$ and (b) its sign function. This is based on the $9^{\text {th }}$ segment of real data in Abbink approach.

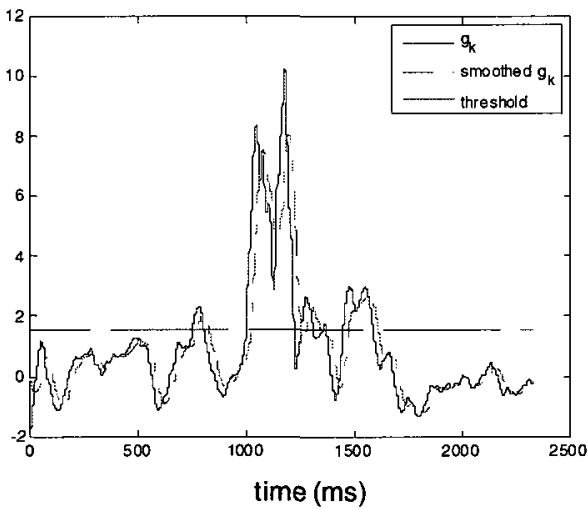

(a)

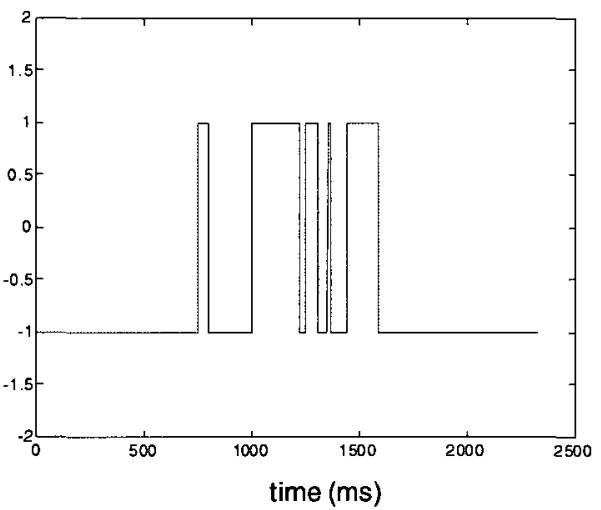

(b)

Figure 6.7 Another example of (a) detection function $g_{k}$ and (b) its sign function. This is based on the $22^{\text {th }}$ segment of real data in Hodges approach. 


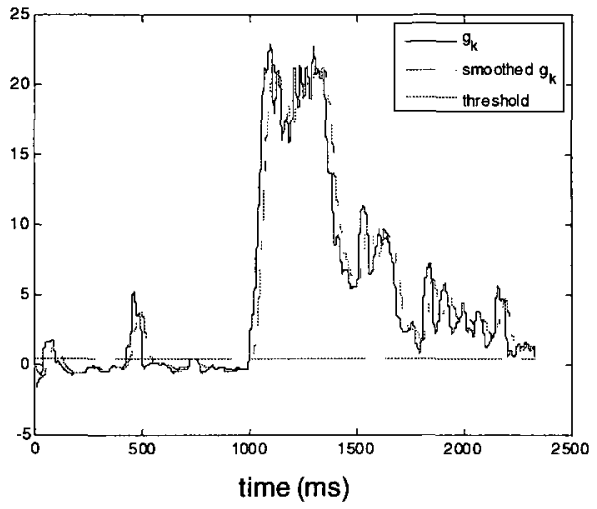

(a)

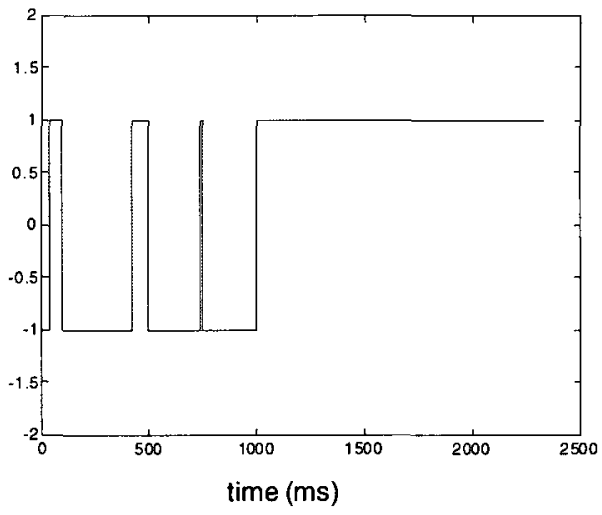

(b)

Figure 6.8 Another example of (a) detection function $g_{k}$ and (b) its sign function. This is based on the $46^{\text {th }}$ segment of real data in Lidierth approach.

In general, the valid sign functions in above scenarios can be grouped into two general cases: the number of ups is equal to that of downs as shown in Figure 6.6 and Figure 6.7, and the number of ups is larger than that of downs as shown in Figure 6.5 and Figure 6.8. In the following figures, we simplify the above scenario into cases. The $\mathrm{x}$-axis represents time $t$ with unit ms, and the $\mathrm{y}$-axis is the amplitude.

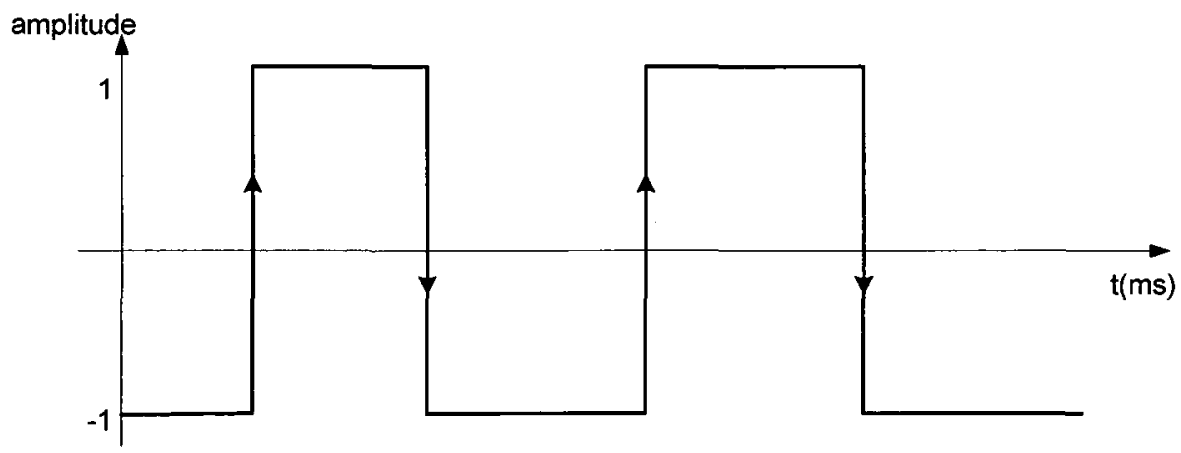

Figure 6.9 Case 1: The number of ups and that of downs are same.

Case 1 has a specific scenario as shown in Figure 6.10, which corresponds to the scenario in Figure 6.6. 


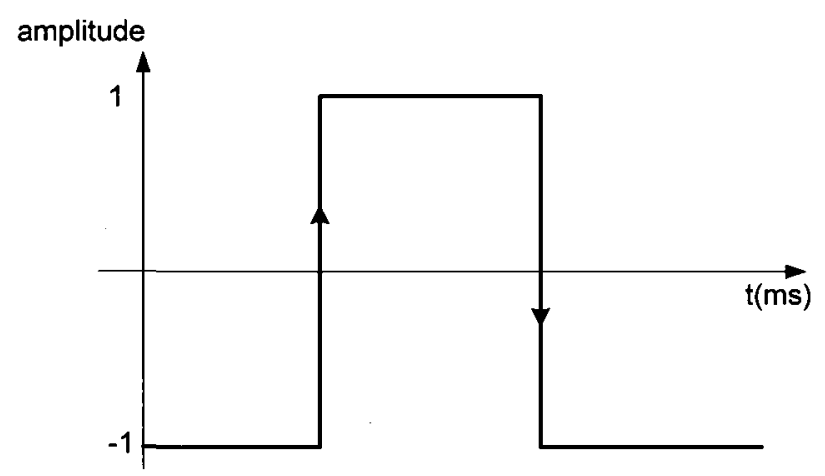

Figure 6.10 A special case of Case 1: The number of ups and that of downs equal to 1.

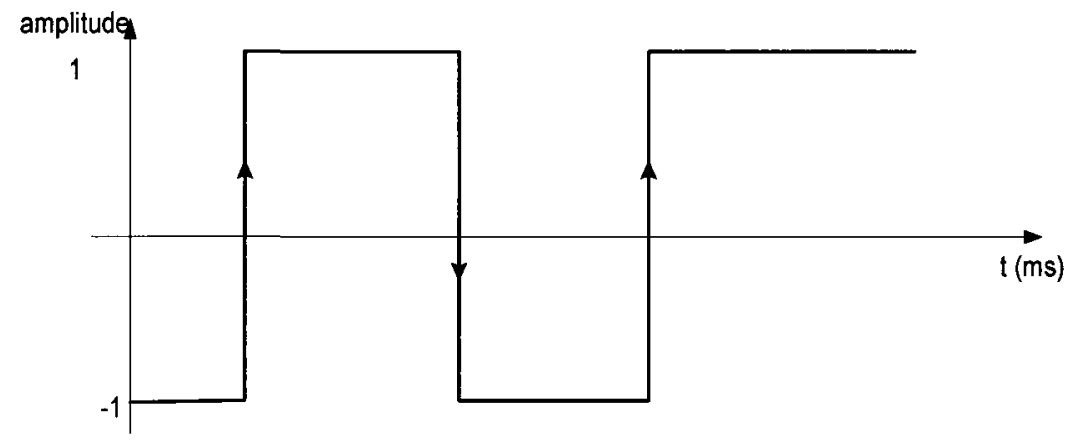

Figure 6.11 Case 2: The number of ups is more than that of downs.

Case 2 also has a specific case during threshold selection as shown in Figure 6.12, which corresponds to the scenario in Figure 6.5.

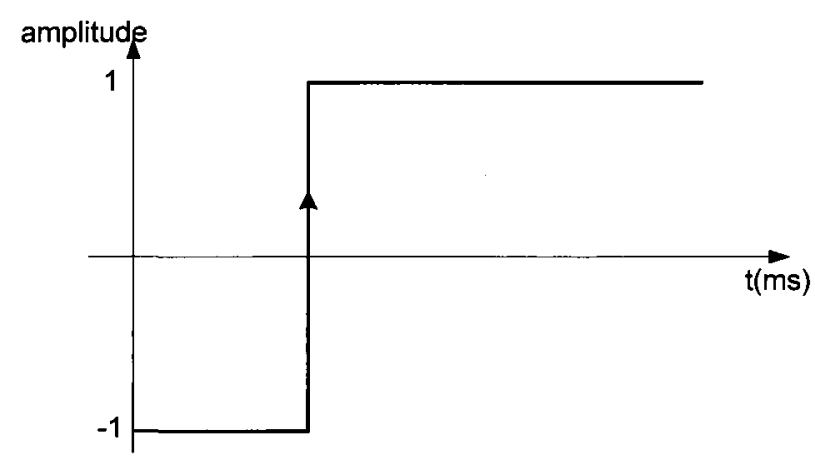

Figure 6.12 A special case of Case 2: The number of ups equals to 1 and that of downs equals to zero. 
Note that we always assume that the threshold has to be larger than the initial part of the noise and the threshold has to be increased. So the following cases shown in Figure 6.13 are invalid and we will discard them during selection.

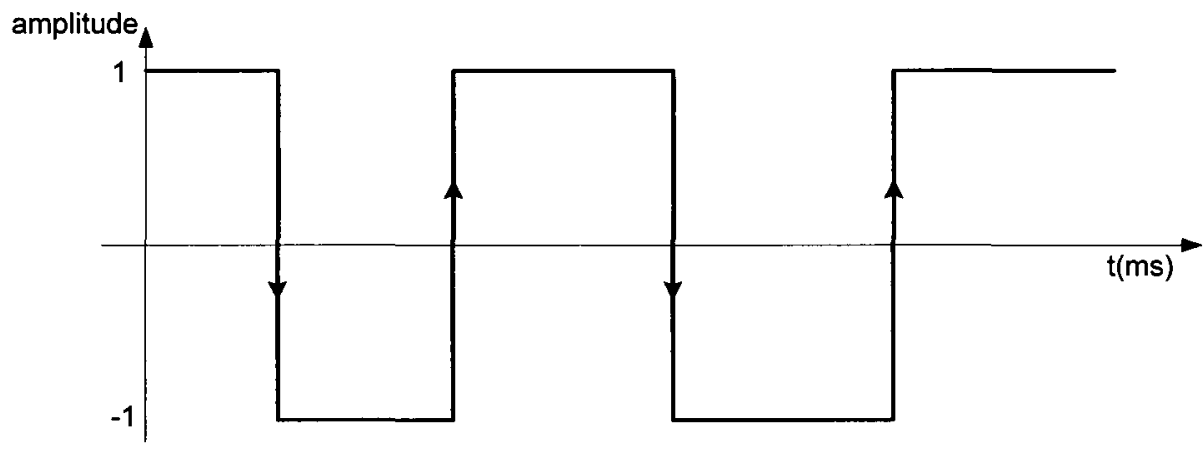

(a)

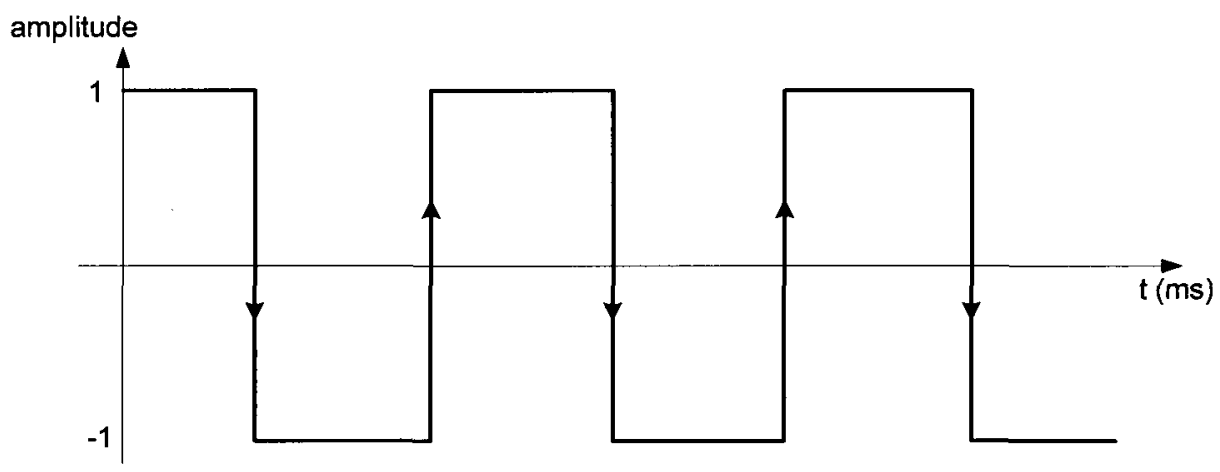

(b)

Figure 6.13 Case 3: Two examples of sign function between detection function and the failed thresholds.

Besides the analysis of detection function and sign function, we also investigate whether it is necessary to add preconditioning system for input data. In Figure 6.14, we give an example of detection function and its sign function in a set of real data using Hodges' approach. 


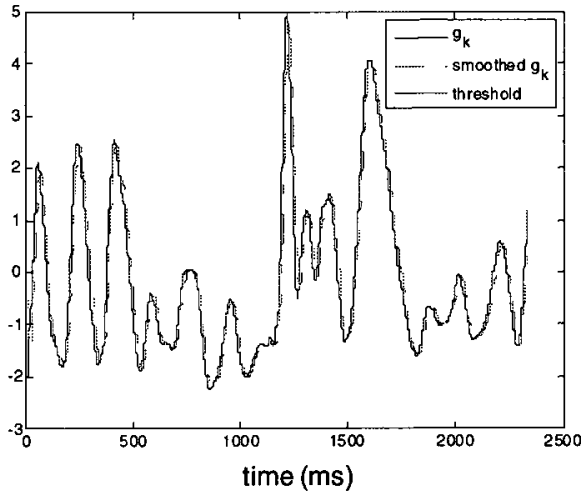

(a)

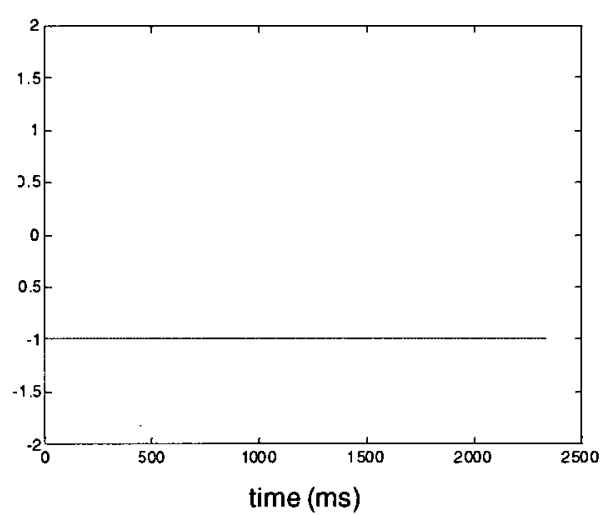

(b)

Figure 6.14 (a) The detection function and (b) its sign function. This is based on the $25^{\text {th }}$ segment real data for Hodges' approach.

To remove low frequency noises, we apply a $6^{\text {th }}$ order Butterworth HPF with $20 \mathrm{~Hz}$ cutoff frequency to this set of data. The processed signal has more detectable noise to signal transition than the unprocessed one, as shown in Figure 6.15.

Before applying the HP filter, as shown in Figure 6.14, it is hard to select a threshold for the detection function $g_{k}$ to select onset time. However, after applying the HP filter, the detection function $g_{k}$ is shown in Figure 6.16 (a) and the sign function shown in Figure 6.16 (b) belongs to one of the general cases we concluded. Thus, we should apply the optimal HP filter, as determined in Chapter 5, for each set of real data before going through the threshold selection algorithm. 


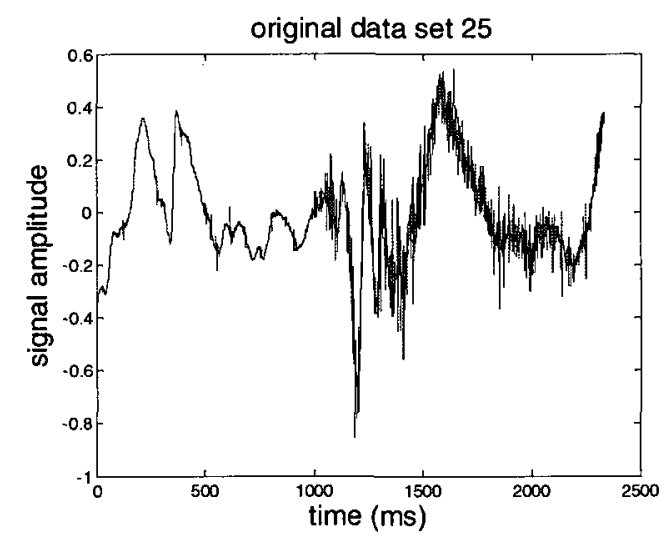

(a)

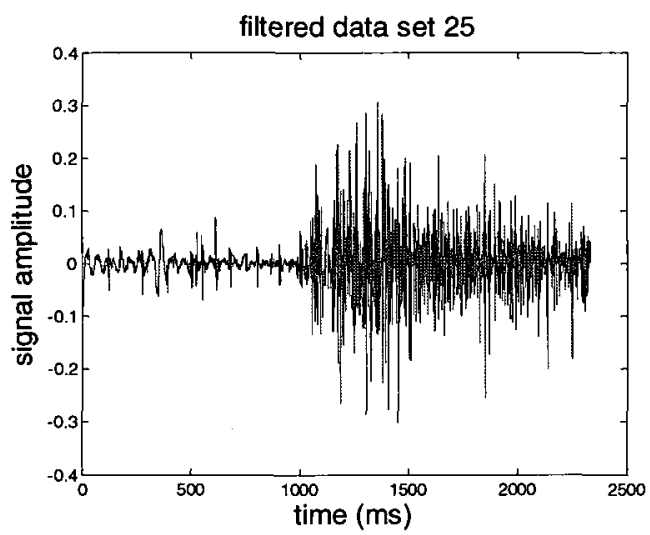

(b)

Figure 6.15 Comparison between (a) raw signal and (b) high pass filtered $25^{\text {th }}$ set data.

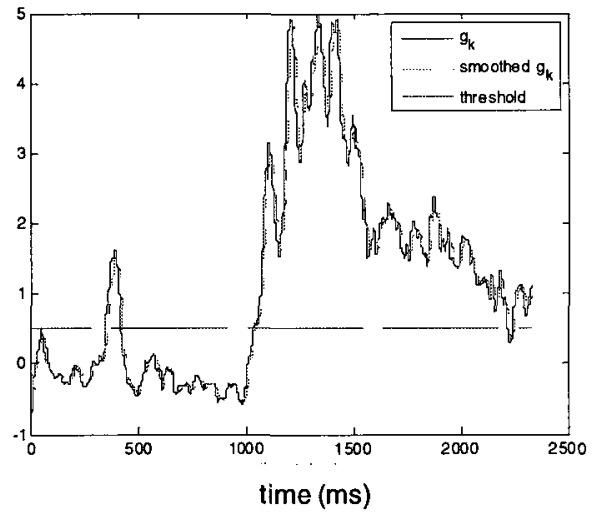

(a)

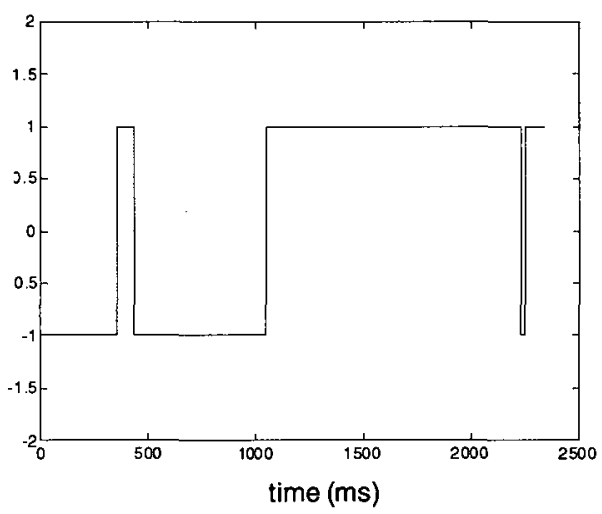

(b)

Figure 6.16 (a) Detection function $\mathrm{g}_{\mathrm{k}}$ of Hodges' approach using the HP filtered signal and (b) the sign function of $\mathrm{g}_{\mathrm{k}}$.

\subsection{Algorithm Details}

As we stated earlier, the threshold-selection scheme should be applicable to various onset detectors/estimators, and thus, is a function of a general onset detection function.

We set a range for threshold $h$, and use $\min \_h$ and $\max \_h$ to represent the minimum and maximum threshold, respectively. The threshold based approaches in the literature 
usually use absolute thresholds and we can use either absolute ones or relative ones. If the threshold is relative ones, we normalize the decision function $g_{k}$ to make its maximum value as max_h.

For each threshold $h$ ranging from $\min \_h$ to $\max \_h$, we proceed the procedures shown in Figure 6.17. 
During initialization, set counter $=0$,

1. Pass an onset detector/estimator algorithm into this function, and call decision function $g_{k}$ using the candidate threshold $h(i i)$.

varargin $\{2\}=h(i i) ;\left[t o(i i), g_{k}\right]=$ fhandle(varargin $\left.\{:\}\right)$;

active_len $=0$;

2. Get the sign function $\tilde{g}$ of $\hat{g}$ by comparing $\hat{g}$ with threshold $h(i i)$.

$\tilde{g}=\operatorname{sign}(\hat{g}-\mathrm{h}(i \mathrm{i}))$;

3. Use ind ${ }_{n e g}$ to record the index of $\hat{g}$ that is smaller than the threshold and ind pos to record the index of $\widehat{g}$ that is higher than the threshold.

4. Verify whether both ind ${ }_{p o s}$ and $i n d_{n e g}$ exist and are larger than a certain value. This is to prevent the case that threshold $h(i i)$ is larger than all value in $\hat{g}$ or smaller than all value in $\hat{g}$ and to reduce false alarm due to outliers.

5. If the above criterion is met, we apply a filter with coefficients [1 -1] to the sign function to find the location and count the number of Ups and Downs.

6. If the number of Ups is larger than that of Downs, consider the location of the last Downs as the length of the sign function. If the number of Ups is less than that of Downs, continue.

7. Use max(location_Dns - location_Ups) to find the longest ones in sign function.

- If there is only one Ups, this is Case 1 and active_len $=1$.

- If the longest chunk of ones is also the first chunk of ones, active_len $=1$.

- If the very first chunk of ones is close to the longest chunk of ones, active_len $=1$. Note that this criterion only applies to multi-threshold approaches with post-processors.

8. If the longest chunk of ones is long enough and active_len $=1$, counter++. If counter is larger than ones, select threshold $h(i i)$ as optimal threshold and exit.

Figure 6.17 Optimal threshold decision algorithm.

\subsection{Results \& Analysis}

With the above analysis, we apply this threshold selection scheme to both synthetic and real data. 


\subsubsection{Results using Synthetic Data}

We apply the approach in Figure 6.17 to synthetic data and show the performance of the four threshold-based approaches using optimal threshold in this section. The performance comparison is conducted using the synthetic data and Figure 6.18 shows that the performance is improved comparing to Figure 4.7.

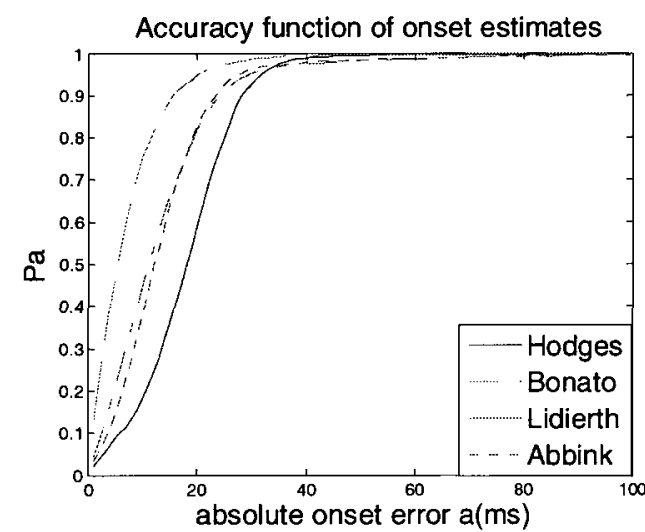

(a)

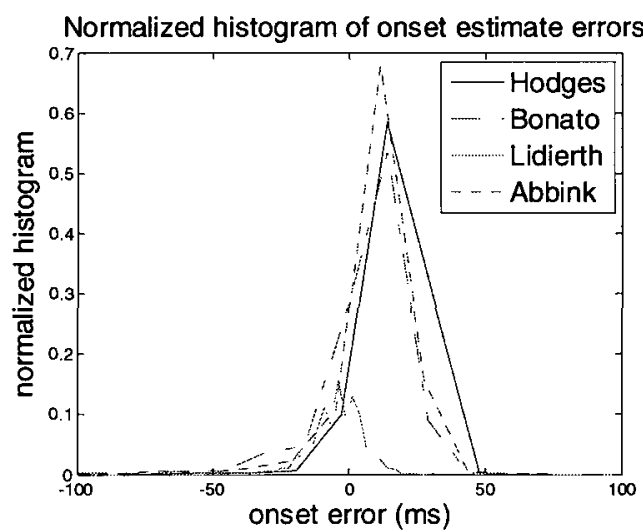

(b)

Figure 6.18 (a) Accuracy function and (b) normalized histograms of detection error of four approaches with optimal threshold on synthetic data.

We also show the mean and standard deviation value in Table 6.1.

Table 6.1: More metrics on detection error for four approaches on synthetic data.

\begin{tabular}{ccccc}
\hline Approaches & Hodges & Bonato & Lidierth & Abbink \\
\hline \hline Mean error(ms) & 16 & 8 & -4 & 8 \\
S.T.D. error (ms) & 27 & 25 & 10 & 28 \\
Skewness (ms) & 5.22 & 5.20 & 2.10 & 6.18 \\
Detection rate (\%) & $96.90 \%$ & $100 \%$ & $98.90 \%$ & $99.15 \%$ \\
P $_{100}$ & 0.9974 & 0.9950 & 1.0000 & 0.9950 \\
\hline
\end{tabular}


Comparing to the results w/o optimal threshold shown in Figure 4.7 and Table 4.1 in Section 4.3.3, all approaches have much smaller standard deviation of detection error and higher value of $\mathrm{P}_{100}$ by using the optimal threshold. Although the mean error of Abbink approach is slightly increased from 4 to 8 , it is not that important since mean error is only a bias and can be fixed easily.

\subsubsection{Results using Real SEMG Data}

As we discussed earlier, the detection function $g_{k}$ of real SEMG data is much more complicated than that of synthetic data. In this section, we first evaluate the performance of the optimal threshold algorithm without HP filtering the input data; then we combine HPF with the threshold selection algorithm, and show their performance.

Here, we show some example of the optimal threshold on each approach using the $2^{\text {nd }}$ segment real data, which has onset time decided by human inspector as $1010 \mathrm{~ms}$ (with $10 \mathrm{~ms}$ transition time).

The subfigures (a) in these figure show how to decide the optimal threshold and the subfigures (b) show the relation between threshold and the detected onset time. We may notice that the title of subfigures (b) give the optimal threshold and the corresponding detected onset time. 


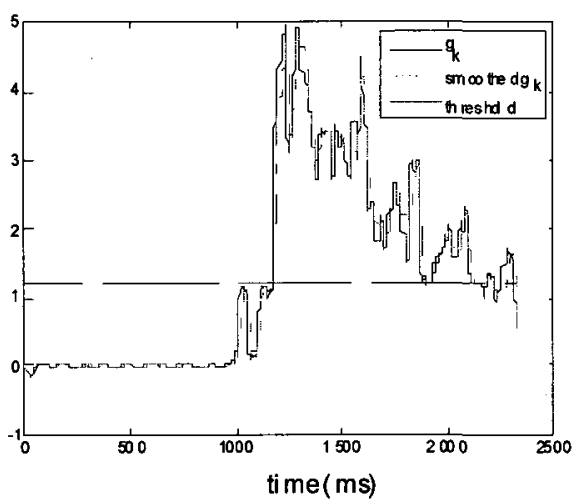

(a)

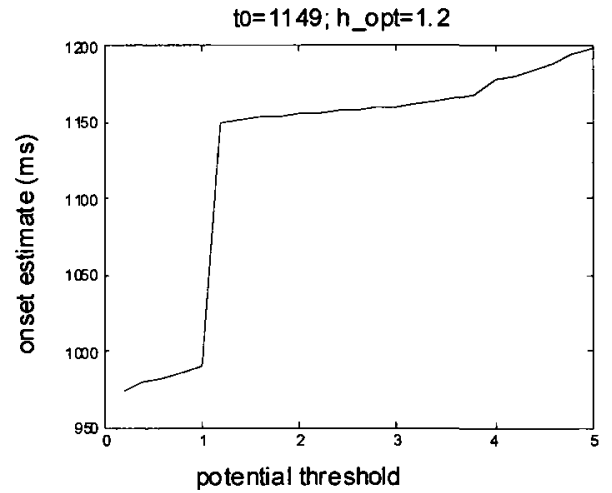

(b)

Figure 6.19 (a) Optimal threshold selection and (b) the relation between the detected onset time and thresholds in Hodge's approach, with detected onset time 1149 ms.

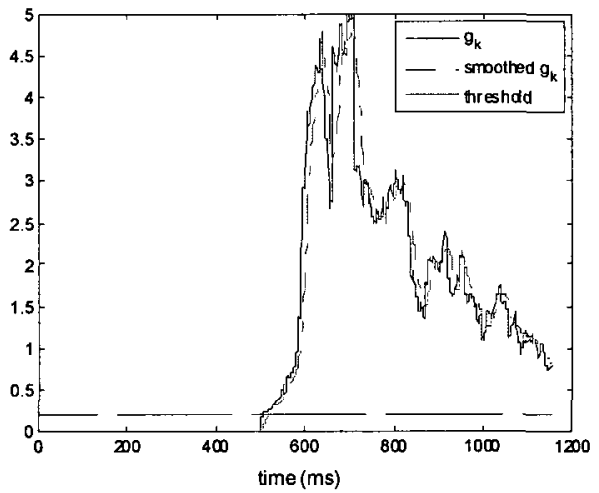

(a)

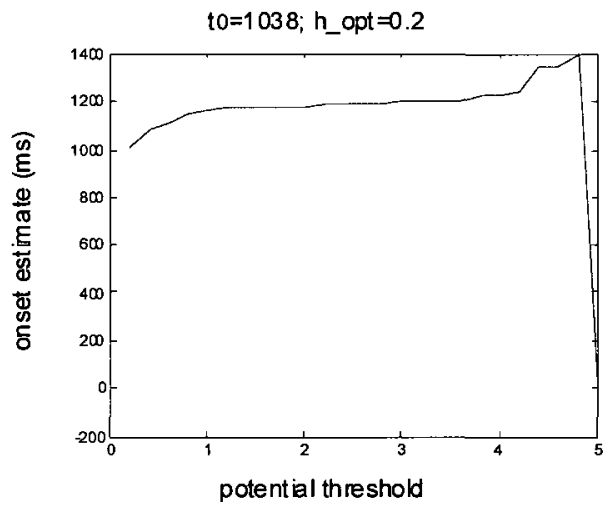

(b)

Figure 6.20 (a) Optimal threshold selection and (b) the relation between the detected onset time and thresholds in Bonato's approach, with detected onset time $1038 \mathrm{~ms}$. 


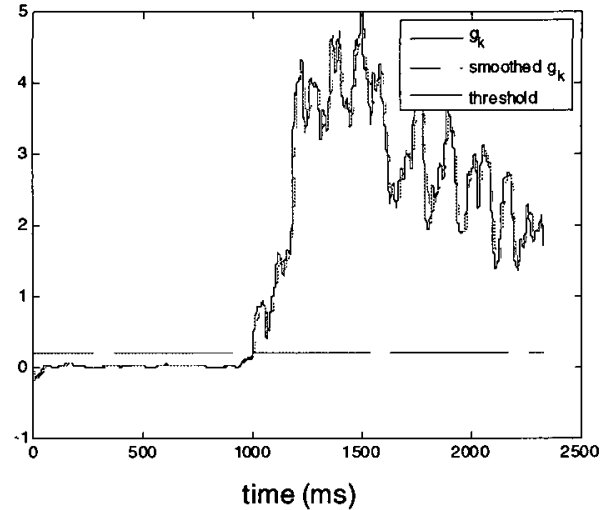

(a)

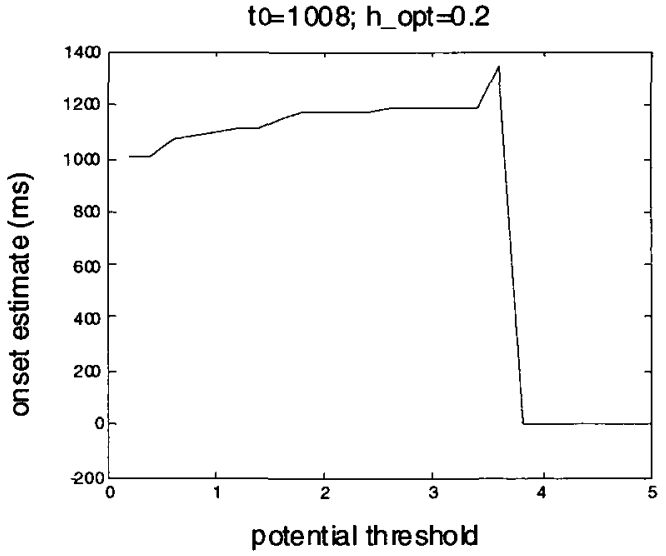

(b)

Figure 6.21 (a) Optimal threshold selection and (b) the relation between the detected onset time and thresholds in Lidierth's approach, with detected onset time $1008 \mathrm{~ms}$.

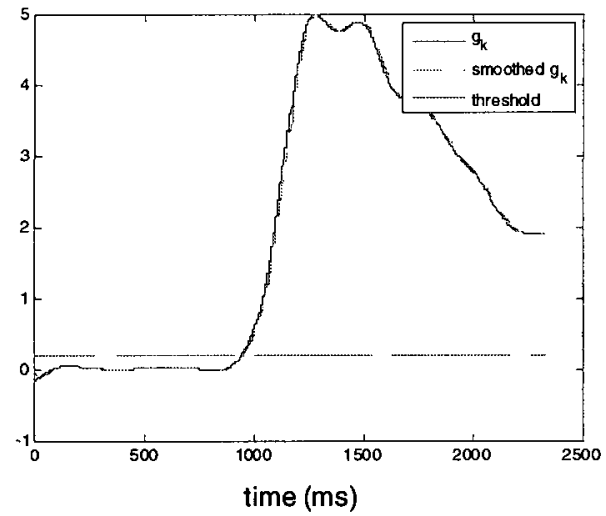

(a)

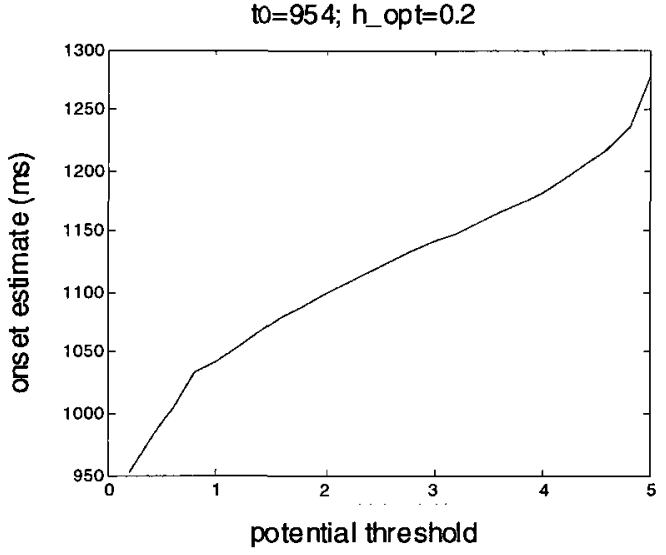

(b)

Figure 6.22 (a) Optimal threshold selection and (b) the relation between the detected onset time and thresholds in Abbink's approach, with detected onset time 954 ms.

We evaluate our algorithm without HP filtering input data, as shown in Figure 6.23. 


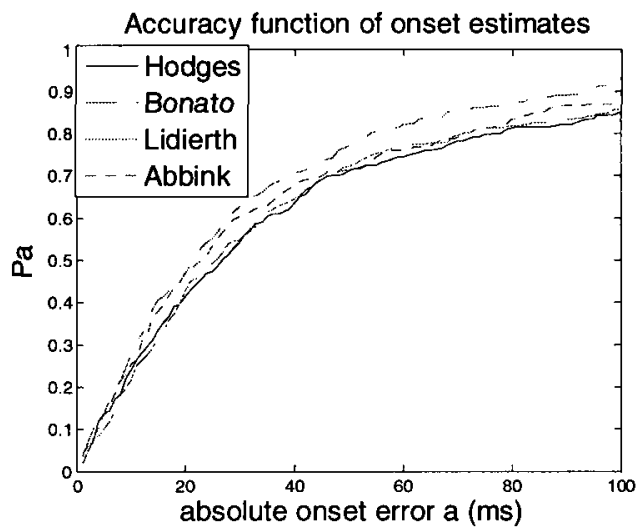

(a)

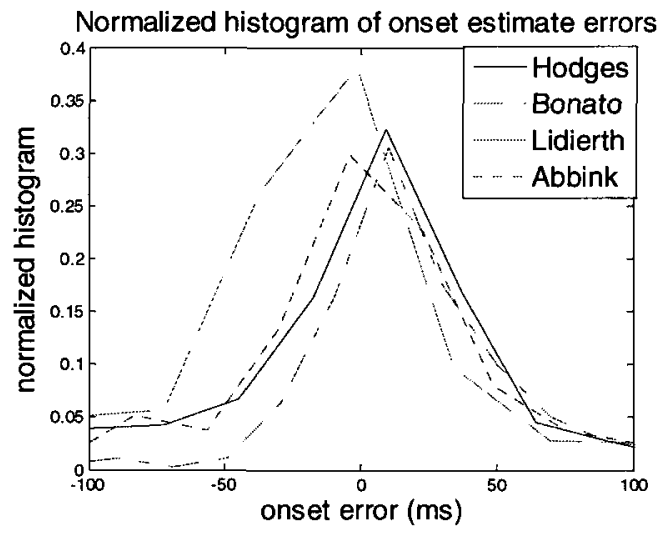

(b)

Figure 6.23 (a) Accuracy function and (b) normalized histograms of detection error of four approaches using optimal threshold w/o HPF on real data.

The mean, standard deviation and other metrics of these four approaches without HPF are shown in Table 6.2 .

Table 6.2: More metrics on detection error using optimal threshold w/o HPF.

\begin{tabular}{ccccc}
\hline Approaches & Hodges & Bonato & Lidierth & Abbink \\
\hline \hline Mean error(ms) & 9 & 28 & -8 & 13 \\
Error S.T.D.(ms) & 129 & 80 & 135 & 117 \\
Skewness (ms) & 4.09 & 3.72 & 4.48 & 3.82 \\
Detection rate (\%) & $91.94 \%$ & $100 \%$ & $88.61 \%$ & $97.78 \%$ \\
P & 0.8520 & 0.9111 & 0.8558 & 0.8722
\end{tabular}

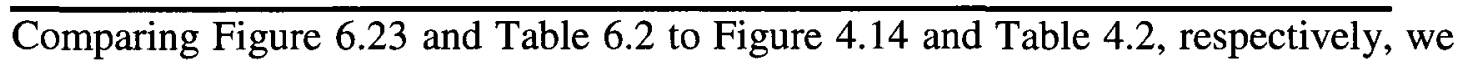
can see that the new results have lower mean error and lower s.t.d. value for all approaches. Also, the value of $\mathrm{P}_{100}$ is higher in the new results for all approaches. Thus, the optimal threshold selection mechanism does improve onset detect accuracy for both single and double threshold methods. 
We then add the HPF into the preconditioning system, with optimal cutoff frequency empirically found in Section 5.3; that is, $410 \mathrm{~Hz}$ for Hodges approach, $60 \mathrm{~Hz}$ for Bonato approach, $250 \mathrm{~Hz}$ for Lidierth approach, and $380 \mathrm{~Hz}$ for Abbink approach. The accuracy function and normalized histogram are shown in Figure 6.24.

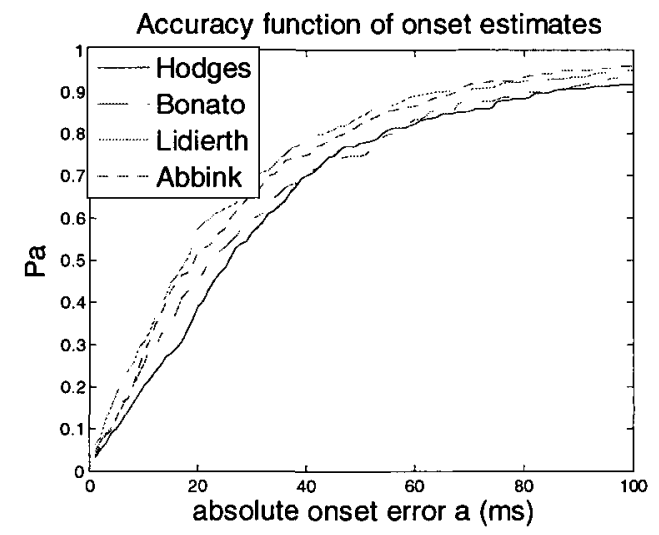

(a)

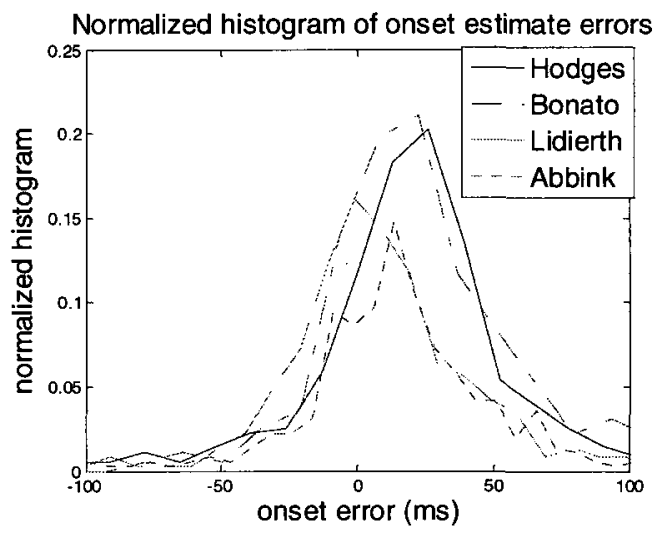

(b)

Figure 6.24 (a) Accuracy function and (b) normalized histograms of detection error of four approaches using optimal threshold with HPF on real data.

Table 6.3 also shows the mean, standard deviation and other metrics of these four approaches using the real data.

Table 6.3: More metrics on detection error using optimal threshold with HPF.

\begin{tabular}{ccccc}
\hline Approaches & Hodges & Bonato & Lidierth & Abbink \\
\hline Mean error(ms) & 8 & 29 & 7 & 13 \\
Error S.T.D.(ms) & 68 & 56 & 48 & 42 \\
Skewness (ms) & 2.97 & 2.93 & 2.36 & 2.12 \\
Detection rate (\%) & $98.61 \%$ & $100 \%$ & $99.72 \%$ & $100 \%$ \\
$\mathrm{P}_{100}$ & 0.9183 & 0.9361 & 0.9499 & 0.9611 \\
\hline
\end{tabular}


Since we apply HP filter to real data, we compare our results with Table 5.5. During the comparison, we found that the value of $\mathrm{P}_{100}$ and the detection rate of the new approach are comparable to that in Table 5.5, which are already quite good. We also found that the mean error of these approaches is quite similar to those in Table 5.5 except the mean error of Abbink is reduced a lot in the new results.

As we mentioned earlier in Section 4.3.2, the mean of error is bias and is easy to remove. Thus, standard deviation of the error is more important. And the standard deviations of detection error in Table 6.2 are much less than those in Table 5.5 for all approaches, which is comparable to the manual onset detection of $34 \mathrm{~ms}$.

From the above analysis, we can see that the HPF does contribute to the performance improvement of the optimal threshold algorithm.

\subsection{Conclusion}

In this chapter, we develop an optimal threshold selection approach and evaluate its performance with and without combining with HPF.

In the results without using HPF as a preconditioning system, we noticed that the mean error, standard deviation, $\mathbf{P}_{100}$, and detection rate are all improved via comparing Figure 6.23 and Table 6.2 to the results in Section 4.4.1. We also compare the results with HPF included to the results in Section 5.3. As shown in Figure 6.24 and Table 6.3, the performance of all approaches has been improved compared with the results in Section 5.3. While the mean error and standard deviation of Hodge's approach is much smaller in the new results, those of other three approaches are quite similar to the result in 
Table 5.5. This is because the other three approaches have post processor that does not need such tight constraint during optimal threshold selection.

Thus, the optimal threshold benefits the Hodge's approach most, which is due to the difference between single-threshold and double/multiple-threshold approaches. We consider the difference during the algorithm development. For example, Hodges' approach is very sensitive to the threshold and during the threshold selection, thus we have to guarantee the noise value before the onset time is lower than the threshold. However, this kind of safe alarm time also tends to overestimate the onset time. The other three approaches have post-processors, which means we can set alarm time with looser constraints on the threshold. Once the alarm time is set, the post-processor can select the estimated onset time.

Based on the above analysis, we conclude that an optimal threshold helps improve the performance of onset detectors. In addition, we found that the high pass filter is very helpful as a pre-conditioning procedure to remove unwanted low frequency signal in real data. 


\section{Chapter 7 Conclusions and Future Work}

\subsection{Conclusions}

This work is focused on the onset detection for SEMG signals. It has three major contributions: 1) reviewing common SEMG signal models and analyzing popular threshold-based onset detection approaches using real SEMG signals and synthetic data in Chapter 4 (the performance of original detection approaches has been improved with our modification); 2) investigating the influence of high pass filtering to the onset detection accuracy in Chapter 5 ; 3) proposing a mechanism to determine the optimal threshold for threshold-based onset detection approaches in Chapter 6.

Based on our research, we conclude that threshold-based onset detectors are good for real-time processing. Among these threshold-based approaches, the double or multiple threshold approaches usually have better performance than those single-threshold one. However, the freedom of double thresholds also increases the vulnerability in parameter settings for these approaches.

During our research, we also find that high pass filtering helps improve the performance of the onset detectors. Nevertheless, the selection of its cutoff frequency is very important and directly affects the detection accuracy.

In addition, a good threshold is the key factor to the success of these threshold-based approaches. However, this kind of study has been severely ignored in current research. 
Threshold-based approaches usually pick up a preset value based on experience and the threshold is an absolute value that does not adapt to signal magnitude. Thus, we propose a mechanism to choose an optimal threshold for each function. Experimental results show that the optimal threshold approach improves the performance of onset detectors on real data but has less impact on synthetic data, due to the fact that synthetic data always has controllable data range and no noise. In addition, pervious methods use absolute threshold while the algorithm could provide a relative or an absolute threshold.

Besides the above contributions, we also conduct research on the modeling of shaping filter and the design of whitening filter in Section 4.1.1. Whitening filter is quite popular in the area of onset detection, due to its capability of removing correlation among signals. Due to the time limit, we do not have chance to compare various design of whitening filters; however, we implement whitening filter in Bonato's approach, which greatly improve its performance comparing to the original results in [64].

The addition of a HPF as well as an optimal threshold selection algorithm has improved the performance of the onset detection methods. The results show that the automated methods have a performance that is comparable to manual detection. Having an accurate automated onset detection method would be beneficial in a variety of applications, reducing the manual workload and reducing the variability between different people. 


\subsection{Future work}

The author suggests the following as potential future work to further this line of research:

1. Investigation of real data collection and synthetic data generation. A real data collection process is very important and directly affects the quality of the collected data. The data used in this thesis were not originally intended for onset detection and were limited to one motion from one muscle group. Additional data would further prove the generalizability of the methods. Also, analysis of real data helps develop a good model to generate synthetic data that describes the structure and characteristics of the actual data. Since synthetic data are widely applied in evaluating onset detection approaches, we can not emphasize the importance of the model enough. Models that include the effects of noise may provide better insight in how to make the methods more robust.

2. Investigation of manual onset detection. Manual onset detection in this work was only performed by a single person with experience in EMG analysis. Having multiple people analyze the data would enable a better understanding of the variability in manual onset detection. It is expected that there would be higher inter-person variability compared to intra-person variability. Demonstrating this would strengthen the argument for utilizing automated methods. 
3. Analysis of adaptive whitening filter and its effect to the onset detection accuracy. The whitening filter used in this study is non-adaptive, which can not adapt to different noise level. To develop an adaptive whitening filter, we should add an adaptive Wiener filter to address the additive noise problem introduced in the non-adaptive whitening filter and an adaptive gain correction system, which could be done in future work.

4. Developing a complete real-time onset time detection system. Based on the analysis and study in this work, the author actually have some preliminary thoughts about developing a onset time detection scheme, which includes a HPF or whitening filter, the optimal threshold selection algorithm, and a post processor. Development would also include real-time considerations, including method complexity and processing delay time. 


\section{References}

[1] J. H. Abbink, A. van der Bilt, and H. W. van der Glas, "Detection of onset and termination of muscle activity in surface electromyograms," J.Or al Rhabil, vol. 25, pp. 365-369, 1998.

[2] G. Barrett, H. Shibasaki, and R. Neshige, "A computer-assisted method for averaging movement-related cortical potentials with respect to EMG onset," Electroenceph. Clin. Neurophysiol., vol. 60, pp. 276-281, 1985.

[3] J. V. Basmajian and C. J. De Luca, Muscle alive, Baltimore: Williams and Wilkins, 1985.

[4] B. Bigland-Ritchie, "EMG/force relations and fatigue of human voluntary contractions," Exer. Sport Sci. Rev., vol. 9, pp. 75-117, 1981.

[5] P. Bonato, T. D'Alessio, and M. Knaflitz, "A statistical method for the measurement of muscle activation intervals from surface myoelectric signal during gait," IEEE Trans.on Biomed. Engineering, vol. 45, pp. 287-298, 1998.

[6] R. A. Bogey, L. A. Barnes, and J. Perry, "Computer algorithms to characterize individual subject EMG profiles during gait," Arch. Phys. Med. Rehabil, vol. 73, pp. 835-851, Sept. 1992.

[7] J. Botwinick and L. W. Thompson, "Premotor and motor componentsof reaction time," Journal of Experimental Psychology, vol. 71, no.1, pp. 9-15, 1966.

[8] P. Brodin, T. S. Miles, and K. S. Turker, "Simple reactiontime responses to mechanical and electrical stimuli in human masseter muscle," Arch.Or al Biol., vol. 38, pp. 221-226, 1993.

[9] G. Brody, R. N. Scott, and R. Balasubramanian, "A model for myoelectric signal generation," Med. Biol. Eng., vol. 12, pp. 29-41, 1974.

[10]G. J. M. Van Boxtel, L. H. D. Geraats, M. M. C. Van den Berg-Lenssen, and C. H. M. Brunia, "Detection of EMG onset in ERP research", Psychophysiology, vol. 30, pp. 405-412, 1993. 
[11]E. A. Clancy and N. Hogan, "Single site electromyograph amplitude estimation," IEEE Trans. BME vol. 41, pp. 159-167, 1994.

[12]E. A. Clancy and K. A. Farray, "Adaptive whitening of the electromyogram to improve amplitude estimation," IEEE Trans. Biomed. Eng., vol. 47, pp. 709-719, 2000.

[13]E. A. Clancy, E. L. Morin, and R. Merletti, "Sampling, noise reduction and amplitude estimation issues in surface electromyography," J Electromyogr Kinesiol., vol. 12, pp. 1-16, 2002.

[14]M. Coles, G. Gratton, T. Bashore, C. Eriksen, and E. Donchin, "A psychophysiological investigation of the continuous flow model of human Information processing," Journal of Experimental Psychology: Human Perception and Performance, pp. 529-553, 1985

[15]T. D'Alessio, N. Accornero, and A. Berardelli, "Toward a real time adaptive processor for surface EMG signals," Ann. Int. Conf. IEEE Eng. Med. Biol. Soc., vol. 9, pp. 323-324, 1987.

[16]C. J. De Luca, "Physiology and mathematics of myoelectric signals," IEEE Trans. on Biomedical Engineering, vol. 26, pp. 313-325, 1979.

[17] G. V. Dimitrov and N. A. Dimitrova, "Precise and fast calculation of the motor unit potentials detected by a point and rectangular plate electrode," Med. Eng. Phys., vol. 20, pp. 374-381, 1998.

[18]C. Disselhorst-Klug, J. Silny, and G. Rau, "Improvement of spatial resolution in surface-EMG: A theoretical and experimental comparison of different spatial filters," IEEE Trans. BME., vol. 44, pp. 567-574, 1997.

[19] J. Ekstedt and E. Stålberg, "How the size of the needle electrode leading-off surface influences the shape of the single muscle fibre action potential in electromyography," Comput Programs Biomed., vol. 3, pp. 204-212, 1973.

[20]K. B. Englehart, "Manifestations of non-stationary neural discharge statistics in myoelectric signal feature estimation," M.S. thesis, University of New Brunswick, NB, Canada, 1992.

[21] K. B. Englehart and P. A. Parker, "Single motor unit myoelectric signal analysis with nonstationary data," IEEE Trans. BME., vol. 41, pp. 168-180, 1994. 
[22]D. Farina and R. Merletti, "Comparison of algorithms for estimation of EMG variables during voluntary isometric contractions," Journal of Electromyography and Kinesiology, vol. 10, pp. 337-349, 2000.

[23]D. Farina and R. Merletti, "A novel approach for precise simulation of the EMG signal detected by surface electrodes," IEEE Trans. on Biomedical Engineering, vol. 48, pp. 637-646, 2001.

[24] A. J. Fuglevand, D. A. Winter, and A. E. Patla, "Models of recruitment and rate coding organization in motor-unit pools," Journal Neurophysiol, vol. 70, pp. 2470$2488,1993$.

[25] A. W. Gaillard and J. Perdok, "Slow brain potentials in the CNV-paradigm". Acta Psychotogica, vol. 44, pp. 147-163, 1980.

[26] T. H. Gootzen, D. F. Stegeman, and A. Van Oosterom, "Finite limb dimensions and finite muscle length in a model for the generation of electromyographic signals," Electroencephalogr Clin. Neurophysiol., vol. 81, pp. 152-162, 1991.

[27] A. Goge and A. D. C. Chan, Investigating classification parameters for continuous myoelectrically controlled prostheses, 28th Conference of the Canadian Medical \& Biological Engineering Society, Quebec City, Canada, pp.141-144, 2004.

[28]M. I. Harba and P. A. Lynn, "Optimizating the acquisition and processing of surface electromyographic signals," Journal of biomedical engineering, vol. 3, pp. 100-106, 1981.

[29]J. N. Helal and P. Bouissou, "The spatial integration effect of surface electrode detecting myoelectric signal". IEEE Trans. on Biomedical Engineering, vol. 39, no. 11, pp. 1161-1167, 1992.

[30]E. Henneman and L. M. Mendell, "Functional organization of the motoneuron pool and its inputs," in V. B. Brooks, ed., Handbook of physiology: The nervous system, American Physiological Society, Bethesda, 1981, pp. 423-507.

[31]P. W. Hodges and B. H. Bui, "A comparison of computer-based methods for determination of onset of muscle contraction using electromyography," Electroenceph.Clin. Neurophysiol, vol.101, pp. 511-519, 1996.

[32]I. S. Hwang and L. D. Abraham, "Quantitative EMG analysis to investigate synergistic coactivation of ankle and knee muscles during isokinetic ankle movement. Part 1: time amplitude analysis," Journal of Electromyography and Kinesiology, vol. 11, pp. 319-325, 2001. 
[33] V. T. Inman, H. J. Ralston, J. B. Saunders, B. Feinstein, and E. W. Wright, "Relation of human electromyogram to muscular tension," Electroenceph. Clin. Neurophysiol., vol. 4, pp. 187-194, 1952.

[34]L. B. Jackson, Digital Filters and Signal Processing, Second Edition, Boston, MA: Kluwer Academic Publishers, 1989 , pp. 255-257.

[35] J. Jessop and O. C. J. Lippold, "Altered synchronization of motor unit firing as a mechanism for long-lasting increases in the tremor of human hand muscles following brief, strong effort," Journal of Physiology, 269, pp. 29-30, 1977.

[36]H. Kranz and G. Baumgartner, "Human alpha motorneurone discharge, a statistical analysis," Brain Res., vol. 67, pp. 324-329, 1974.

[37]P. J. Lago and N. B. Jones, "Effect of motor unit firing time statistics on EMG spectra," Medical Biology Engineering \& Computer, vol.15, pp. 648-655, 1977.

[38] J. H. Lawrence and C. J. De Luca, "Myoelectric signal versus force relationship in different human muscles," Journal of Applied Physiology, vol. 54, pp. 1653-1659, 1983.

[39] J. K. Leader, J. R. Boston, and C. A. Moore, "A data dependent computer algorithm for the detection of muscle activity onset and offset from EMG recordings," Electroencephalography and Clinical Neurophysiology, vol. 109, pp. 119-123, 1998.

[40]M. Lidierth, "A computer based method for automated measurement of the periods of muscular activity from an EMG and its application to locomotor EMGs," Electroencephalography and Clinical Neurophysiology, vol. 64, pp. 378-380, 1986.

[41]L. H. Lindstrom and R. I. Magnusson, "Interpretation of myoelectric power spectra: a model and its applications," Proc. of IEEE, vol. 65, pp. 653-662, 1977.

[42]P. Madeleine, P. Bajaj, K. Sogaard, and L. Arendt-Neilsen, "Mechanomyography and electromyography force relationships during concentric, isometric and eccentric contractions," Journal of Electromyography and Kinesiology, vol. 11, pp. 113-121, 2001.

[43] J. Makhoul, "Linear prediction: A tutorial review," Proc. of IEEE, vol. 63, pp. 561$581,1995$.

[44]R. Merletti and P. A. Parker, Electromyography: Physiology, Engineering, and Noninvasive Applications, Hoboken, NJ: John Wiley \& Sons, Inc., 2004. 
[45] A. Merlo, D. Farina, and R. Merletti, "A Fast and Reliable Technique for Muscle Activity Detection From Surface EMG Signals," IEEE trans. on Biomedical Engineering, vol. 50, no. 3, Mar. 2003.

[46] S. Micera, A. M. Sabatini, and P. Dario, "An algorithm for detecting the onset of muscle contraction by EMG signal processing," Med. Eng. Phys., vol. 20, pp. 211215, 1998.

[47] S. D. Nandedkar, D. B. Sanders, and E. V. Stålberg, "Simulation techniques in electromyography," IEEE Trans. on Biomedical Engineering, vol. 32, pp.775-785, 1985.

[48]J. Nilsson, M. Panizza, and M. Hallet, "Principles of digital sampling of a physiologic signal," Electroencephalography and Clinical Neurophysiology, vol. 89, pp. 349-358, 1993

[49]J. Nilsson, M. Panizza, and P. Arieti, "Computer-aided determination of the silent period," Journal on Clinical Neurophysiology, vol. 14, pp. 136-143, 1997.

[50]M. Pećina, J. Krmpotić-Nemanić, and A. D. Markiewitz, Tunnel syndromes: peripheral nerve compression syndromes, 3rd ed., Boca Raton, FL: CRC Press LLC, 2001.

[51]R. S. Person and L. P. Kudina, "Discharge frequency and discharge pattern in human motor units during voluntary contractions of muscle," Electroencephalography and Clinical Neurophysiology. vol. 32, pp. 47-83, 1972.

[52]H. Reucher, G. Rau, and J. Silny, "Spatial Filtering of Noninvasive Multielectrode EMG: Part I-Introduction to Measuring Technique and Applications," IEEE trans. on Biomedical Engineering, vol. BME-34, no. 2, 1987.

[53]H. Reucher, J. Silny, and G. Rau, "Spatial Filtering of Noninvasive Multielectrode EMG: Part II-Filter Performance in Theory and Modeling," IEEE trans. on Biomedical Engineering, vol. BME-34, no. 2, Feb. 1987.

[54]J. R. Potvin, R. W. Norman, and S. M. McGill, "Mechanically corrected EMG for the continuous estimation of erector spine muscle loading during repetitive lifting," European Journal of Applied Physiology, vol. 74, pp. 119-132, 1996.

[55] J. R. Potvin and S. H. M Brown, "Less is more: high pass filtering, to remove up to 99\% of the surface EMG signal power, improves EMG-based biceps brachii muscle force estimates," Journal of Electromyography and Kinesiology, vol. 14, pp. 389$399,2004$. 
[56]D. Popivanov, "Computer detection of EMG edges for synchronization of movement-related brain potentials," Electroencephalography and Clinical Neurophysiology, 64. 171-176, 1986.

[57]J. G. Proakis and D. G. Manolakis, Digital Signal Processing: Principles, Algorithms, and Applications, 3rd ed., Upper Saddle River, NJ: Prentice Hall, 1996.

[58]R. Shiavi and M. Negin, "Stochastic properties of motoneuron activity and the effect of muscular length," Biol. Cybern., vol. 19, pp. 231-237, 1975.

[59]E. Shwedyk, R. Balasubramanian, and R. N. Scott, "A non-stationary model of the electromyogram," IEEE Trans. BME., vol. 24, pp. 417-424, 1977.

[60]E. V. Stålberg and M. Sonoo, "Assessment of variability in the shape of the motor unit action potential, the jiggle at consecutive discharges," Muscle \& Nerve, vol. 17, pp. 1135-1144, 1994.

[61]E. Stålberg and B. Falck, "The role of electromyography in neurology," Electroencephalography and Clinical Neurophysiology, vol. 103, pp. 579-598, 1997.

[62] G. Staude and W. Wolf, "Voluntary motor reactions: does stimulus appearance prolong the actual tremor period?" Electromyography and Kinesiology, vol. 9, pp. 277-281, 1999.

[63]G. Staude, R. Dengler, and W.Wolf, "The discontinuous nature of motor execution part I: a model concept for single-muscle multiple-task coordination," Biol.C ybern., vol. 82, pp. 23-33, 2000.

[64]G. Staude, C. Flachenecker, M. Daumer, and W. Wolf, "Onset Detection in Surface Electromyographic Signals: A Systematic Comparison of Methods," Journal on Applied Signal Processing, vol. 2, pp. 67-81, 2001.

[65]M. Talebinejad, "Multi-Scale Analysis of Myoelectric Signals: Chaos and Random Fractal Theory," PhD dissertation, The Ottawa-Carleton Institute for Electrical and Computer Engineering (OCIECE), Ottawa, ON, Canada, 2008

[66] M. Talebinejad, A. D. C. Chan, A. Miri, and R. M. Dansereau, "Fractal analysis of surface electromyography signals: A novel power spectrum-based method", Journal of Electromyography and Kinesiology, 2008.

[67]K. Tegtmeyer, L. Ibsen, and B. Goldstein, "Computer-assisted learning in critical care: From ENIAC to HAL," Crit. Care. Med. Vol. 29, pp. 177-182, 2001. 
[68]Tri-Council Policy Statement: Ethical Conduct for Research Involving Humans, Canada: Public Works and Government Services Canada, 1998.

[69]A. D. Weiss, "The locus of reactloti time change with set, tnotivation, and age," Journal of Gerontology, vol. 20, pp. 60-64, 1965.

[70]D. A. Winter, "Biomechanics of human movement with applications to the study of human locomotion," CRC Crit. Rev. Biomed. Eng, vol. 9, pp. 287-314, 1999.

[71] J. J. Woods and B. Bigland-Ritchie, "Linear and non-linear surface EMG-force relationships in human muscle," Am J Phys Med. vol. 62, pp. 287-299, 1983. 